\title{
Broadening the toolkit for quantitatively evaluating noncanonical amino acid incorporation in yeast
}

Supplementary information

Jessica T. Stieglitz, ${ }^{\dagger}$ Kelly A. Potts, ${ }^{\dagger}$ and James A. Van Deventer ${ }^{\star} \dagger, \ddagger$

*Corresponding author, James.Van_Deventer@tufts.edu

† Chemical and Biological Engineering Department, Tufts University, Medford, Massachusetts 02155, USA

$\ddagger$ Biomedical Engineering Department, Tufts University, Medford, Massachusetts 02155, USA

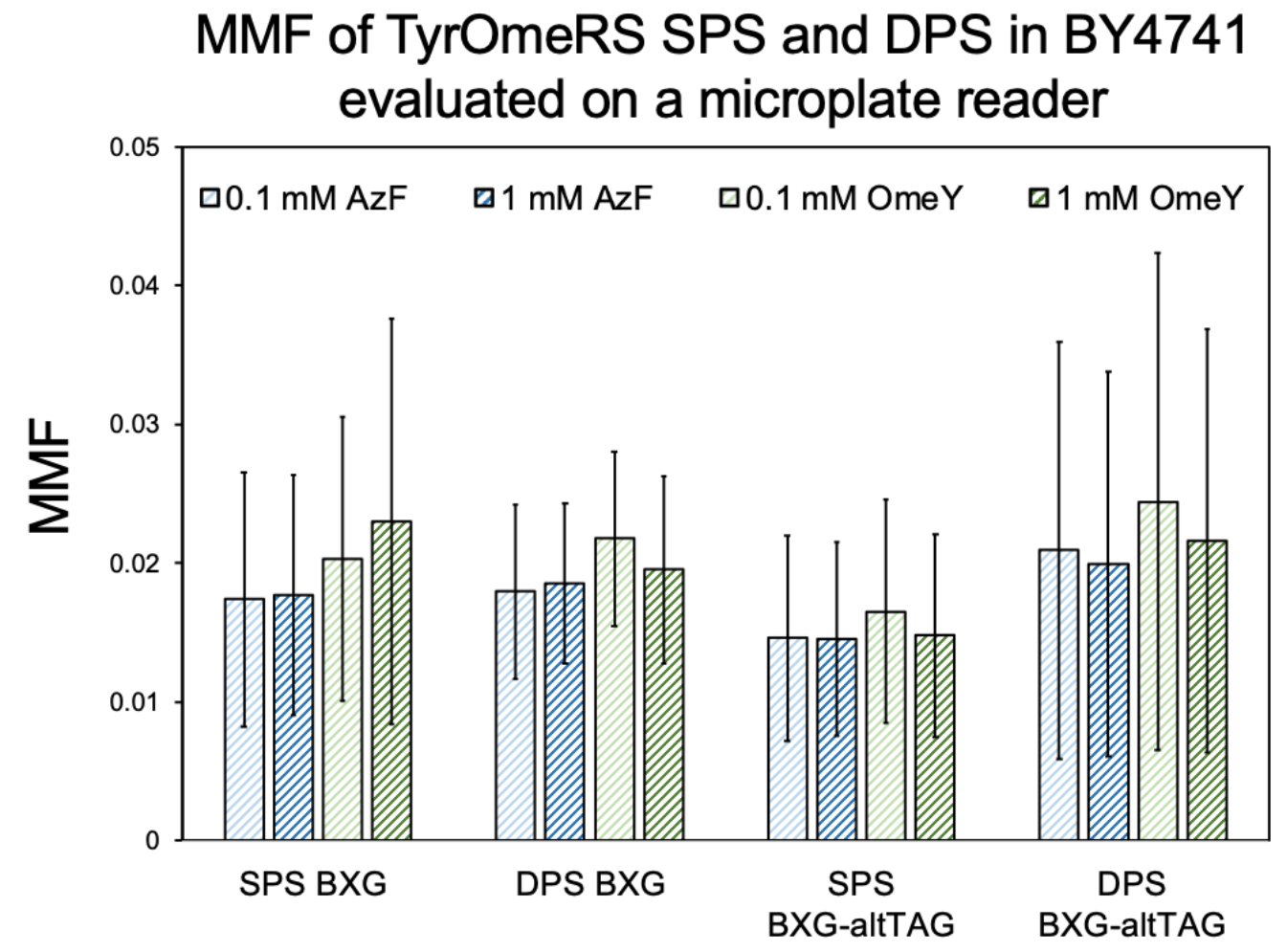

Supplementary Figure 1. Maximum misincorporation frequency (MMF) of SPSs and DPSs with TyrOmeRS and two ncAAs evaluated on a spectrophotometric microplate reader. Both BFPGFP reporters BXG and BXG-altTAG were evaluated with TyrOmeRS. Experiments were performed in biological replicates with $\mathrm{N}=3$. 


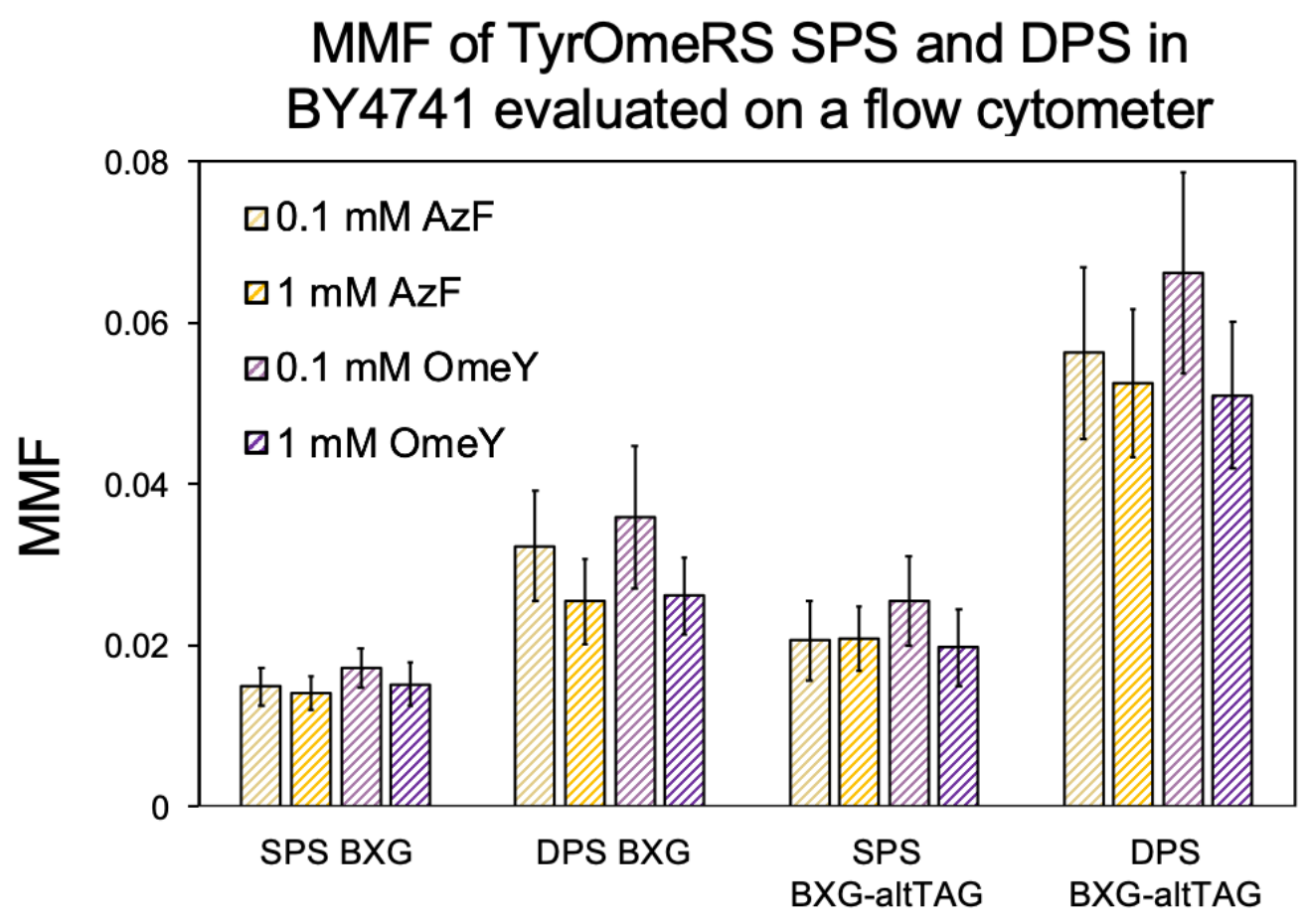

Supplementary Figure 2. Maximum misincorporation frequency (MMF) of SPSs and DPSs with TyrOmeRS and two ncAAs evaluated on a flow cytometer. Both BFP-GFP reporters BXG and BXG-altTAG were evaluated with TyrOmeRS. Experiments were performed in biological replicates with $\mathrm{N}=3$. 


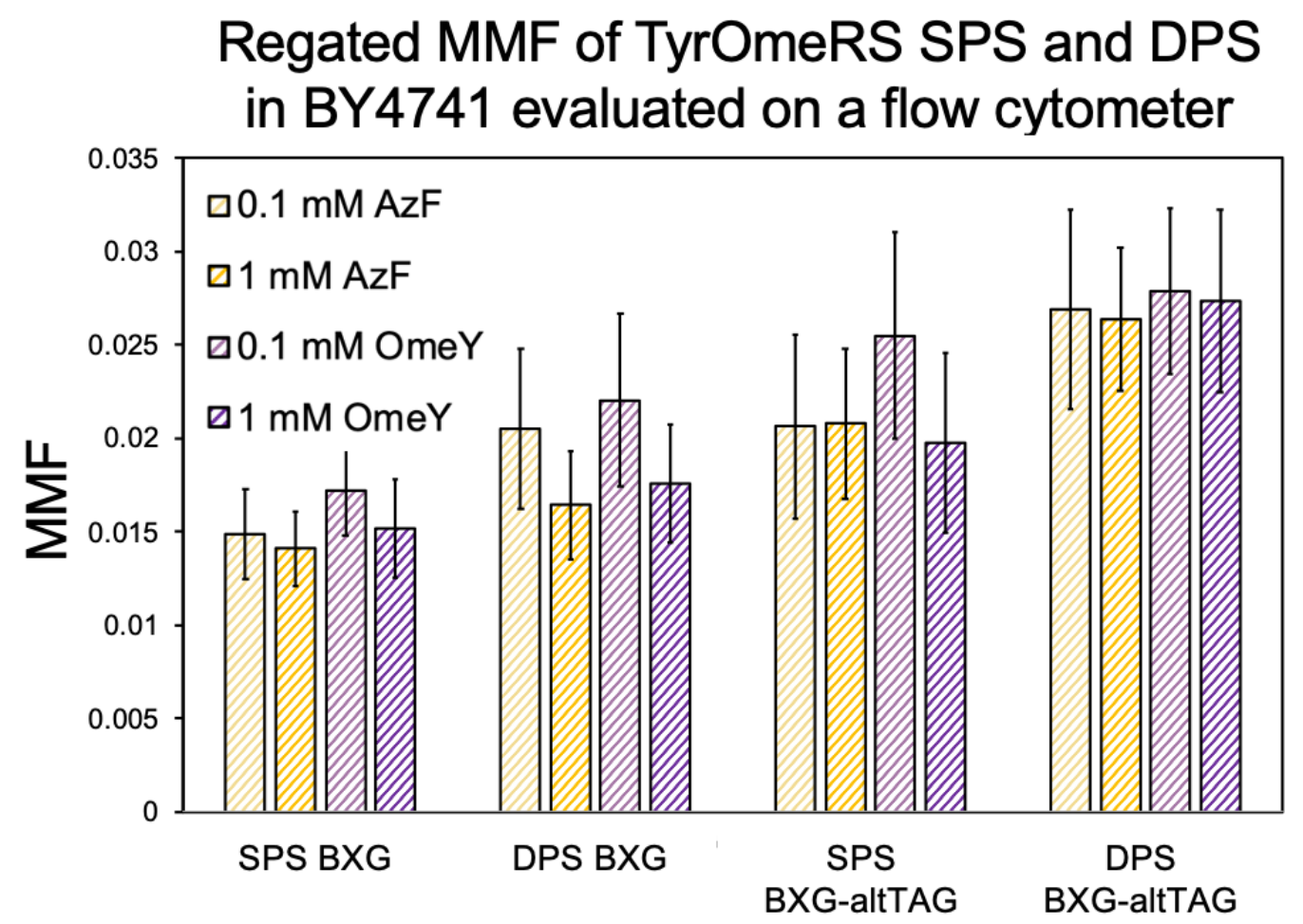

Supplementary Figure 3. Regated maximum misincorporation frequency (MMF) of SPSs and DPSs with TyrOmeRS and two ncAAs evaluated on a flow cytometer. Both BFP-GFP reporters $B X G$ and BXG-altTAG were evaluated with TyrOmeRS. Experiments were performed in biological replicates with $\mathrm{N}=3$. 


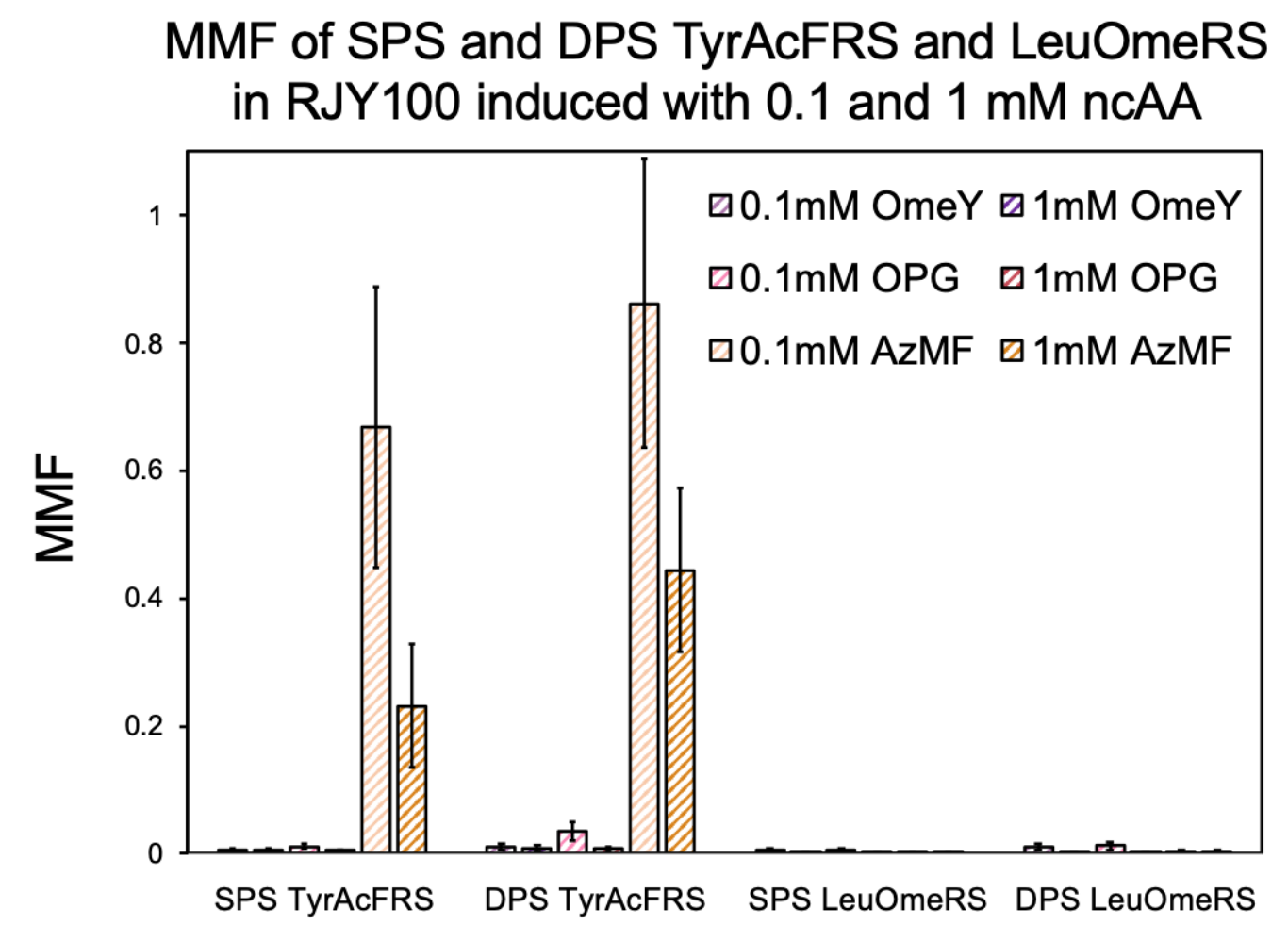

Supplementary Figure 4. Maximum misincorporation frequency (MMF) of SPSs and DPSs with two aaRSs and three ncAAs evaluated on a flow cytometer. Yeast display reporter FAPB2.3.6L1TAG was evaluated with TyrAcFRS and LeuOmeRS. Experiments were performed in biological replicates with $\mathrm{N}=3$. 


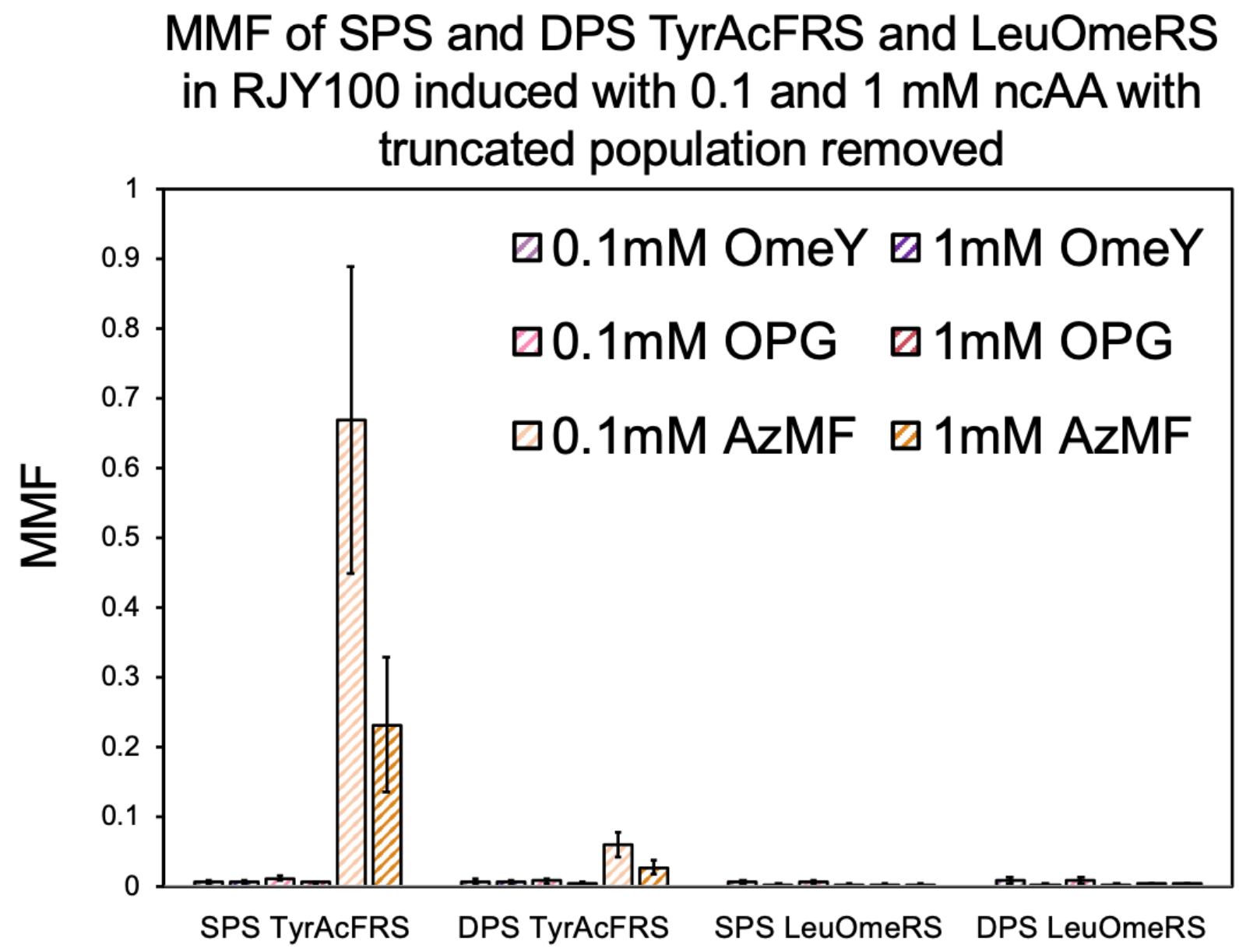

Supplementary Figure 5. Maximum misincorporation frequency (MMF) of SPSs and DPSs with two aaRSs and three ncAAs evaluated on a flow cytometer. Yeast display reporter

FAPB2.3.6L1TAG was evaluated with TyrAcFRS and LeuOmeRS. Experiments were performed in biological replicate with $\mathrm{N}=3$. 


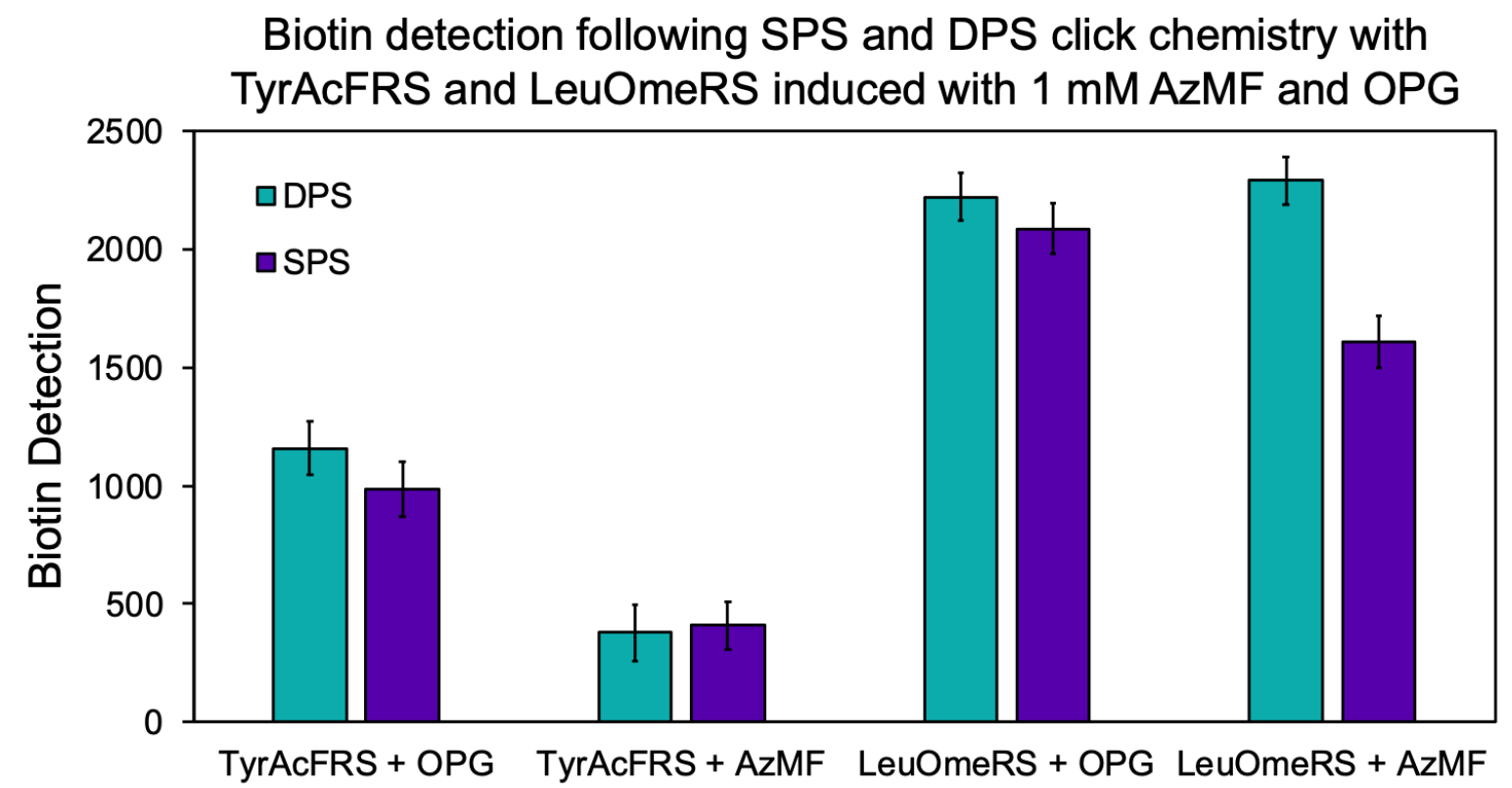

Supplementary Figure 6 . Biotin detection levels of CuAAC click chemistry with SPSs and DPSs for OTSs TyrAcFRS and LeuOmeRS induced with $1 \mathrm{mM} O P G$ and AzMF. Only one technical replicate was used for this experiment, and error bars are coefficient of variation (CV) error exported from FlowJo analysis. This was the first of two CuAAC experiments performed; the second experiment included three technical replicates and the results are reported in the main text. 


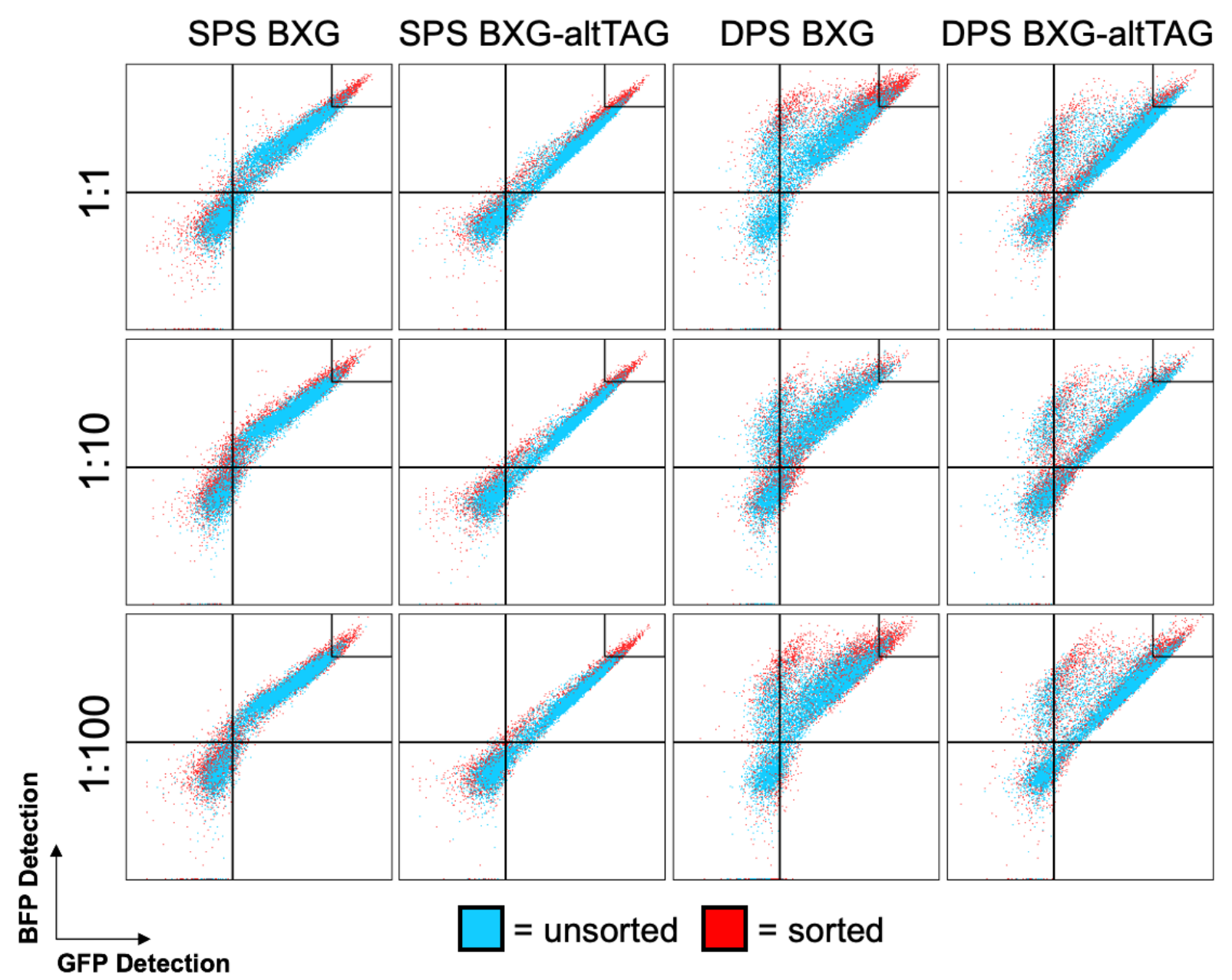

Supplementary Figure 7. Sample representation of gates used to measure cell counts for evaluating enrichment of BY4741 $\triangle \mathrm{PPQ} 1$ from model sorts. Technical replicate 1 shown here out of three technical replicates. 
Supplementary Table 1. List of plasmid combinations transformed or co-transformed into $S$. cerevisiae for SPS versus DPS experiments in the main text.

\begin{tabular}{|l|l|l|}
\hline S. cerevisiae strain & Plasmid 1 & Plasmid 2 \\
\hline RJY100 & pCTCON2-FAPB2.3.6 & pRS315-LeuOmeRS \\
\hline RJY100 & pCTCON2-FAPB2.3.6-LeuOmeRS & N/A \\
\hline RJY100 & pCTCON2-FAPB2.3.6L1TAG & pRS315-LeuOmeRS \\
\hline RJY100 & pCTCON2-FAPB2.3.6L1TAG-LeuOmeRS & N/A \\
\hline RJY100 & pCTCON2-FAPB2.3.6 & pRS315-TyrAcFRS \\
\hline RJY100 & pCTCON2-FAPB2.3.6-TyrAcFRS & N/A \\
\hline RJY100 & pCTCON2-FAPB2.3.6L1TAG & pRS315-TyrAcFRS \\
\hline RJY100 & pCTCON2-FAPB2.3.6L1TAG-TyrAcFRS & N/A \\
\hline BY4741 & pRS416-BYG & pRS315-TyrOmeRS \\
\hline BY4741 & pRS416-BYG-TyrOmeRS & N/A \\
\hline BY4741 & pRS416-BXG & pRS315-TyrOmeRS \\
\hline BY4741 & pRS416-BXG-TyrOmeRS & N/A \\
\hline BY4741 & pRS416-BXG-altTAG & pRS315-TyrOmeRS \\
\hline BY4741 & pRS416-BXG-altTAG-TyrOmeRS & N/A \\
\hline BY4741 & pRS416-BXG & pRS315-LeuOmeRS \\
\hline BY4741 & pRS416-BXG-LeuOmeRS & N/A \\
\hline BY4741 & pRS416-BXG-altTAG & pRS315-LeuOmeRS \\
\hline BY4741 & pRS416-BXG-altTAG-LeuOmeRS & N/A \\
\hline
\end{tabular}


Supplementary Table 2. Median fluorescence intensity (MFI) of WT SPSs and DPSs displayed on the surface of yeast for C-terminus (C-term) and N-terminus (N-term) detection. The autofluorescence (Q4) of each population's N- and C-terminus detection was subtracted prior to averaging sample MFI values. The $\mathrm{N}$-term \% is the AVG SPS background-subtracted ( $\mathrm{N}$-term Q4) MFI over the AVG DPS background-subtracted MFI. Calculations proceeded similarly for Cterminus MFI evaluation.

\begin{tabular}{|c|c|c|c|c|c|c|c|c|c|c|}
\hline Sample & $\begin{array}{c}\text { MFI C- } \\
\text { term }\end{array}$ & $\begin{array}{c}\text { MFI N- } \\
\text { term }\end{array}$ & $\begin{array}{c}\text { MFI C- } \\
\text { term Q4 }\end{array}$ & $\begin{array}{c}\text { MFI N- } \\
\text { term Q4 }\end{array}$ & N-term -Q4 & AVG & $\mathrm{N}$-term \% & C-term -Q4 & AVG & C-term \% \\
\hline SPS LeuWT no ncAA 1 & 85973 & 3498 & 55 & 196 & 3302 & 3231 & & 85918 & 76994.63 & \\
\hline SPS LeuWT no ncAA 2 & 71130 & 3463 & 69 & 173 & 3290 & & & 71061 & & \\
\hline SPS LeuWT no ncAA 3 & 74078 & 3292 & 73.1 & 191 & 3101 & & & 74004.9 & & \\
\hline SPS TyrWT no ncAA 1 & 81166 & 3440 & 69.9 & 181 & 3259 & 3413.67 & & 81096.1 & 81788.97 & \\
\hline SPS TyrWT no ncAA 2 & 74581 & 3371 & 75.1 & 188 & 3183 & & & 74505.9 & & \\
\hline SPS TyrWT no ncAA 3 & 89840 & 4006 & 75.1 & 207 & 3799 & & & 89764.9 & & \\
\hline SPS LeuWT 1 mM OPG 1 & 85973 & 3558 & 75.1 & 188 & 3370 & 3460.67 & & 85897.9 & 79928.63 & \\
\hline SPS LeuWT 1 mM OPG 2 & 76888 & 3668 & 72.1 & 191 & 3477 & & & 76815.9 & & \\
\hline SPS LeuWT 1 mM OPG 3 & 77149 & 3731 & 76.9 & 196 & 3535 & & & 77072.1 & & \\
\hline SPS TyrWT 1 mM OPG 1 & 80892 & 3731 & 73.1 & 198 & 3533 & 3111.67 & & 80818.9 & 69514.57 & \\
\hline SPS TyrWT 1 mM OPG 2 & 75598 & 3558 & 75.1 & 189 & 3369 & & & 75522.9 & & \\
\hline SPS TyrWT 1 mM OPG 3 & 52275 & 2624 & 73.1 & 191 & 2433 & & & 52201.9 & & \\
\hline SPS LeuWT 1 mM AzMF 1 & 78731 & 3247 & 76.1 & 192 & 3055 & 3025.33 & & 78654.9 & 70206.9 & \\
\hline SPS LeuWT 1 mM AzMF 2 & 57277 & 2865 & 72.1 & 185 & 2680 & & & 57204.9 & & \\
\hline SPS LeuWT 1 mM AzMF 3 & 74834 & 3546 & 73.1 & 205 & 3341 & & & 74760.9 & & \\
\hline SPS TyrWT 1 mM AzMF 1 & 71856 & 3558 & 72.1 & 194 & 3364 & 3327.33 & & 71783.9 & 73761.6 & \\
\hline SPS TyrWT 1 mM AzMF 2 & 67380 & 3035 & 79 & 194 & 2841 & & & 67301 & & \\
\hline SPS TyrWT 1 mM AzMF 3 & 82273 & 3978 & 73.1 & 201 & 3777 & & & 82199.9 & & \\
\hline DPS LeuWT no ncAA 1 & 103563 & 4680 & 77.9 & 202 & 4478 & 4611 & 1.427112 & 103485.1 & \begin{tabular}{|l|}
105804.4 \\
\end{tabular} & 1.37418 \\
\hline DPS LeuWT no ncAA 2 & 102517 & 4464 & 76.9 & 206 & 4258 & & & 102440.1 & & \\
\hline DPS LeuWT no ncAA 3 & 111569 & 5305 & 80.9 & 208 & 5097 & & & 111488.1 & & \\
\hline DPS TyrWT no ncAA 1 & 129924 & 5754 & 77.9 & 229 & 5525 & 4969 & 1.45562 & 129846.1 & 116388.3 & 1.42303 \\
\hline DPS TyrWT no ncAA 2 & 106767 & 4664 & 76.1 & 221 & 4443 & & & 106690.9 & & \\
\hline DPS TyrWT no ncAA 3 & 112708 & 5163 & 80.1 & 224 & 4939 & & & 112627.9 & & \\
\hline DPS LeuWT $1 \mathrm{mM}$ OPG 1 & 89840 & 3912 & 77.9 & 215 & 3697 & 4365 & 1.261318 & 89762.1 & 96960.7 & 1.21309 \\
\hline DPS LeuWT 1 mM OPG 2 & 94519 & 4602 & 80.9 & 227 & 4375 & & & 94438.1 & & \\
\hline DPS LeuWT $1 \mathrm{mM}$ OPG 3 & 106767 & 5251 & 85.1 & 228 & 5023 & & & 106681.9 & & \\
\hline DPS TyrWT 1 mM OPG 1 & 116194 & 5305 & 79 & 235 & 5070 & 4365.33 & 1.402892 & 116115 & 101666.6 & 1.46252 \\
\hline DPS TyrWT 1 mM OPG 2 & 94200 & 4404 & 75.1 & 213 & 4191 & & & 94124.9 & & \\
\hline DPS TyrWT 1 mM OPG 3 & 94840 & 4046 & 80.1 & 211 & 3835 & & & 94759.9 & & \\
\hline DPS LeuWT $1 \mathrm{mM}$ AzMF 1 & 94519 & 4172 & 75.1 & 216 & 3956 & 3851 & 1.272918 & 94443.9 & 95995.93 & 1.36733 \\
\hline DPS LeuWT 1 mM AzMF 2 & 90145 & 3820 & 80.1 & 210 & 3610 & & & 90064.9 & & \\
\hline DPS LeuWT 1 mM AzMF 3 & 103563 & 4214 & 84 & 227 & 3987 & & & 103479 & & \\
\hline DPS TyrWT 1 mM AzMF 1 & 106046 & 4494 & 82 & 248 & 4246 & 4405.33 & 1.323983 & 105964 & 99331.93 & 1.34666 \\
\hline DPS TyrWT 1 mM AzMF 2 & 88632 & 4272 & 80.1 & 233 & 4039 & & & 88551.9 & & \\
\hline DPS TyrWT 1 mM AzMF 3 & 103563 & 5180 & 83.1 & 249 & 4931 & & & 103479.9 & & \\
\hline
\end{tabular}


Supplementary Table 3. Fold enrichment values for model sorts of BY4741 and BY4741 $\triangle P P Q 1$ based on colony counts post-sort. Fold enrichment represents the fraction of BY4741 $\triangle P P Q 1$ over BY4741 in the post-sort populations divided by the fraction of BY4741 $\triangle \mathrm{PPQ} 1$ over BY4741 in the pre-sort populations.

\begin{tabular}{|c|c|c|c|}
\hline & \multicolumn{3}{|c|}{ Fold Enrichment } \\
\hline Ratio & $1: 1$ & $1: 10$ & $1: 100$ \\
\hline SPS BXG & 1.5 & 1.9 & 0 \\
\hline SPS BXG-altTAG & 2 & 1.9 & 6 \\
\hline DPS BXG & 1.76 & 3.1 & 0 \\
\hline DPS BXG-altTAG & 1.88 & 8.8 & 6 \\
\hline
\end{tabular}

Supplementary Table 4. Summary of single plasmid system plasmids used in this work.

\begin{tabular}{|c|c|c|c|}
\hline SPS & Parent vector & Insert & Insert vector \\
\hline $\begin{array}{l}\text { pRS416-BYG- } \\
\text { LeuOmeRS** }\end{array}$ & pRS416-BYG & LeuOmeRS/tRNAcuA ${ }^{\text {Leu }}$ & pRS315-LeuOmeRS \\
\hline $\begin{array}{l}\text { pRS416-BXG- } \\
\text { LeuOmeRS }\end{array}$ & pRS416-BXG & LeuOmeRS/tRNA $A_{c u A}^{\text {Leu }}$ & pRS315-LeuOmeRS \\
\hline $\begin{array}{l}\text { pRS416-BXG-altTAG- } \\
\text { LeuOmeRS }\end{array}$ & $\begin{array}{l}\text { pRS416-BXG- } \\
\text { altTAG }\end{array}$ & LeuOmeRS/tRNA $_{c u A}{ }^{\text {Leu }}$ & pRS315-LeuOmeRS \\
\hline $\begin{array}{l}\text { pRS416-BYG- } \\
\text { TyrOmeRS }\end{array}$ & pRS416-BYG & TyrOmeRS/tRNA ${ }_{C \cup A}{ }^{\text {Tyr }}$ & pRS315-OmeRS \\
\hline $\begin{array}{l}\text { pRS416-BXG- } \\
\text { TyrOmeRS }\end{array}$ & pRS416-BXG & TyrOmeRS/tRNA ${ }_{C u A}{ }^{\top y r}$ & pRS315-OmeRS \\
\hline $\begin{array}{l}\text { pRS416-BXG-altTAG- } \\
\text { TyrOmeRS }\end{array}$ & $\begin{array}{l}\text { pRS416-BXG- } \\
\text { altTAG }\end{array}$ & TyrOmeRS/tRNA ${ }_{C \cup A}{ }^{\text {Tyr }}$ & pRS315-OmeRS \\
\hline $\begin{array}{l}\text { pCTCON2-FAPB2.3.6- } \\
\text { LeuOmeRS }\end{array}$ & $\begin{array}{l}\text { pCTCON2- } \\
\text { FAPB2.3.6 }\end{array}$ & LeuOmeRS/tRNAcua ${ }^{\text {Leu }}$ & pRS315-LeuOmeRS \\
\hline $\begin{array}{l}\text { pCTCON2- } \\
\text { FAPB2.3.6L1TAG- } \\
\text { LeuOmeRS }\end{array}$ & $\begin{array}{l}\text { pCTCON2- } \\
\text { FAPB2.3.6 }\end{array}$ & LeuOmeRS/tRNAcua ${ }^{\text {Leu }}$ & pRS315-LeuOmeRS \\
\hline $\begin{array}{l}\text { pCTCON2-FAPB2.3.6- } \\
\text { TyrAcFRS }\end{array}$ & $\begin{array}{l}\text { pCTCON2- } \\
\text { FAPB2.3.6L1TAG }\end{array}$ & TyrAcFRS/tRNA $_{c u A}{ }^{\text {Tyr }}$ & $\begin{array}{l}\text { pRS315-AcFRS- } \\
\text { Res }\end{array}$ \\
\hline $\begin{array}{l}\text { pCTCON2- } \\
\text { FAPB2.3.6L1TAG- } \\
\text { TyrAcFRS }\end{array}$ & $\begin{array}{l}\text { pCTCON2- } \\
\text { FAPB2.3.6L1TAG }\end{array}$ & TyrAcFRS/tRNA ${ }_{c u A}{ }^{\text {Tyr }}$ & $\begin{array}{l}\text { pRS315-AcFRS- } \\
\text { Res }\end{array}$ \\
\hline
\end{tabular}

${ }^{*}$ Data with this vector not shown in manuscript; included as reference sequence.

\section{Sequences of single plasmid systems used in this work}

pRS416-BYG-LeuOmeRS 
Note: original restriction enzyme sites Xbal and Sacll were eliminated at 3' and 5' ends intentionally due to presence of both restriction sites in LeuOmeRS-tRNA ${ }_{c u A}{ }^{\text {Leu }}$ insert.

\section{GPD Promoter, LeuOmeRS,}

linker, GFP

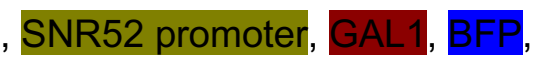

gacgaaagggcctcgtgatacgcctattttataggttaatgtcatgataataatggtttcttaggacggatcgcttgcctgtaacttacacg cgcctcgtatctttaatgatggaataatttgggaatttactctgtgtttatttattttatgtttgtatttggattttagaaagtaaataaagaaggt agaagagttacggaatgaagaaaaaaaaataaacaaaggtttaaaaaatttcaacaaaaagcgtactttacatatatatttattagac aagaaaagcagattaaatagatatacattcgattaacgataagtaaaatgtaaaatcacaggatttcgtgtgtggtcttctacacagac aagatgaaacaattcggcattaatacctgagagcaggaagagcaagataaaaggtagtatttgttggcgatccccctagagtcttttac atcttcggaaaacaaaaactatttttctttaatttcttttttactttctattttaatttatatatttatattaaaaaatttaaattataattatttttatag cacgtgatgaaaaggacccaggtggcacttttcggggaaatgtgcgcggaacccctattgtttattttctaaatacattcaaatatgtat ccgctcatgagacaataaccctgataaatgcttcaataatattgaaaaaggaagagtatgagtattcaacatttccgtgtcgcccttattc cctttttgcggcattttgccttcctgttttgctcacccagaaacgctggtgaaagtaaaagatgctgaagatcagttgggtgcacgagtg ggttacatcgaactggatctcaacagcggtaagatccttgagagtttcgccccgaagaacgtttccaatgatgagcactttaaagttct gctatgtggcgcggtattatcccgtattgacgccggg caagagcaactcggtcgccgcatacactattctcagaatgacttggttgagta ctcaccagtcacagaaaagcatcttacggatggcatgacagtaagagaattatgcagtgctgccataaccatgagtgataacactgc ggccaacttacttctgacaacgatcggaggaccgaaggagctaaccgctttttgcacaacatgggggatcatgtaactcgccttgatc gttgggaaccggagctgaatgaagccataccaaacgacgagcgtgacaccacgatgcctgtagcaatggcaacaacgttgcgca aactattaactggcgaactacttactctagcttcccggcaacaattaatagactggatggaggcggataaagttgcaggaccacttctg cgctcggcccttccggctggctggtttattgctgataaatctggagccggtgagcgtgggtctcgcggtatcattgcagcactggggcca gatggtaagccctcccgtatcgtagttatctacacgacggggagtcaggcaactatggatgaacgaaatagacagatcgctgagata ggtgcctcactgattaagcattggtaactgtcagaccaagtttactcatatatactttagattgatttaaaacttcattttaatttaaaaggatc taggtgaagatccttttgataatctcatgaccaaaatcccttaacgtgagtttcgttccactgagcgtcagaccccgtagaaaagatca aaggatcttcttgagatccttttttctgcgcgtaatctgctgcttgcaaacaaaaaaaccaccgctaccagcggtggtttgtttgccggatc aagagctaccaactcttttccgaaggtaactggcttcagcagagcgcagataccaaatactgtccttctagtgtagccgtagttaggcc accacttcaagaactctgtagcaccgcctacatacctcg ctctgctaatcctgttaccagtggctgctgccagtggcgataagtcgtgtct taccgggttggactcaagacgatagttaccggataaggcgcagcggtcgggctgaacggggggttcgtgcacacagcccagcttgg agcgaacgacctacaccgaactgagatacctacagcgtgagctatgagaaagcgccacgcttcccgaagggagaaaggcggac aggtatccggtaagcggcagggtcggaacaggagagcgcacgagggagcttccagggggaaacgcctggtatctttatagtcctgt cgggtttcgccacctctgacttgagcgtcgattttgtgatgctcgtcaggggggcggagcctatggaaaaacgccagcaacgcggcc ttttacggttcctggccttttgctggccttttgctcacatgttcttcctgcgttatccctgattctgtggataaccgtattaccgcctttgagtga gctgataccgctcgccgcagccgaacgaccgagcgcagcgagtcagtgagcgaggaagcggaagagcgcccaatacgcaaac cgcctctccccgcgcgttggccgattcattaatgcagctggcacgacaggtttcccgactggaaagcgggcagtgagcgcaacgca attaatgtgagttacctcactcattaggcaccccagg ctttacactttatgcttccggctcctatgttgtgtggaattgtgagcggataacaat ttcacacaggaaacagctatgaccatgattacgccaagcgcgcaattaaccctcactaaagggaacaaaagctggagctccaccg cCCTAGGgagctcagtttatcattatcaatactcgccatttcaaagaatacgtaaataattaatagtagtgattttcctaactttatttagt caaaaaattagccttttaattctgctgtaacccgtacatgcccaaaatagggggcgggttacacagaatatataacatcgtaggtgtctg ggtgaacagtttattcctggcatccactaaatataatggagcccgcttttaagctggcatccagaaaaaaaaagaatcccagcacca aaatattgtttcttcaccaaccatcagttcataggtccattctcttagcgcaactacagagaacaggggcacaaacaggcaaaaaac gggcacaacctcaatggagtgatgcaacctgcctggagtaaatgatgacacaaggcaattgacccacgcatgtatctatctcattttctt acaccttctattaccttctgctctctctgatttggaaaaagctgaaaaaaaaggttgaaaccagttccctgaaattattcccctacttgacta ataagtatataaagacggtaggtattgattgtaattctgtaaatctatttcttaaacttcttaaattctacttttatagttagtctttttttagttttaa 
aacaccagaacttagtttcgacggattctagaactagtatggagatttCCATGGAAGAGCAATACCGCCCGGAAGA GATAGAATCCAAAGTACAGCTTCATTGGGATGAGAAGCGCACATTTGAAGTAACCGAAGAC GAGAGCAAAGAGAAGTATTACTGCCTGTCTTTGGAACCCTATCCTTCTGGTCGACTACACA TGGGCCACGTACGTAACTACACCATCGGTGACGTGATCGCCCGCTACCAGCGTATGCTGG GCAAAAACGTCCTGCAGCCGATCGGCTGGGACGCGTTTGGTCTGCCTGCGGAAGGCGCG GCGGTGAAAAACAACACCGCTCCGGCACCGTGGACGTACGACAACATCGCGTATATGAAA AACCAGCTCAAAATGCTGGGCTTTGGTTATGACTGGAGCCGCGAGCTGGCAACCTGTACG CCGGAATACTACCGTTGGGAACAGAAATTCTTCACCGAGCTGTATAAAAAAGGCCTGGTAT ATAAGAAGACTTCTGCGGTCAACTGGTGCCCGAACGACCAGACCGTACTGGCGAACGAAC AAGTTATCGACGGCTGCTGCTGGCGCTGCGATACCAAAGTTGAACGTAAAGAGATCCCGC AGTGGTTTATCAAAATCACTGCTTACGCTGACGAGCTGCTCAACGATCTGGATAAACTGGA TCACTGGCCAGACACCGTTAAAACCATGCAGCGTAACTGGATCGGTCGTTCCGAAGGCGT GGAGATCACCTTCAACGTTAACGACTATGACAACACGCTGACCGTTTACACTACCCGCCCG GACGCTTTTATGGGTTGTACCTACCTGACGGTAGCTGCGGGTCATCCGCTGGCGCAGAAA GCGGCGGAAAATAATCCTGAACTGGCGGCCTTTATTGACGAATGCCGTAACACCAAAGTTG CCGAAGCTGAAATGGCGACGATGGAGAAAAAAGGCGTCGATACTGGCTTTAAAGCGGTTC ACCCATTAACGGGCGAAGAAATTCCCGTTTGGGCAGCAAACTTCGTATTGATGGAGTACGG CACGGGCGCAGTTATGGCGGTACCGGGGCACGACCAGCGCGACTACGAGTTTGCCTCTA AATACGGCCTGAACATCAAACCGGTTATCCTGGCAGCTGACGGCTCTGAGCCAGATCTTTC TCAGCAAGCCCTGACTGAAAAAGGCGTGCTGTTCAACTCTGGCGAGTTCAACGGTCTTGA CCATGAAGCGGCCTTCAACGCCATCGCCGATAAACTGACTGCGATGGGCGTTGGCGAGC GTAAATTGAACTACCGCCTGCGCGACTGGGGTGTTTCCCGTCAGCGTTACTGGGGCGCGC CGATTCCGATGGTGACGCTGGAAGACGGTACCGTAATGCCGACCCCGGACGACCAGCTG CCGGTGATCCTGCCGGAGGATGTGGTAATGGACGGCATTACCAGCCCGATTAAAGCAGAT CCGGAGTGGGCGAAAACTACCGTTAACGGTATGCCAGCACTGCGTGAAACCGACACTTTC GACACCTTTATGGAGTCCTCCTGGAGATATGCGCGCTACACTTGCCCGCAGTACAAAGAA GGTATGCTGGATTCCGAAGCGGCTAACTACTGGCTGCCGGTGGATATCGCTATTGGTGGT ATTGAACACGCCATTATGGGTCTGCTCTACTTCCGCTTCTTCCACAAACTGATGCGTGATG CAGGCATGGTGAACTCTGACGAACCAGCGAAACAGTTGCTGTGTCAGGGTATGGTGCTGG CAGATGCCTTCTACTATGTTGGCGAAAACGGCGAACGTAACTGGGTTTCCCCGGTTGATGC TATCGTTGAACGTGACGAGAAAGGCCGTATCGTGAAAGCGAAAGATGCGGCAGGCCATGA ACTGGTTTATACCGGCATGAGCAAAATGTCCAAGTCGAAGAACAACGGTATCGACCCGCA GGTGATGGTTGAACGTTACGGCGCGGACACCGTTCGTCTGTTTATGATGTTTGCTTCTCCG GCTGATATGACTCTCGAATGGCAGGAATCCGGTGTGGAAGGGGCTAACCGCTTCCTGAAA CGTGTCTGGAAACTGGTTTACGAGCACACAGCAAAAGGTGATGTTGCGGCACTGAACGTT GATGCGCTGACTGAAAATCAGAAAGCGCTGCGTCGCGATGTGCATAAAACGATCGCTAAA GTGACCGATGATATCGGCCGTCGTCAGACCTTCAACACCGCAATTGCGGCGATTATGGAG CTGATGAACAAACTGGCGAAAGCACCAACCGATGGCGAGCAGGATCGCGCTCTGATGCAG GAAGCACTGCTGGCCGTTGTCCGTATGCTTAACCCGTTCACCCCGCACATCTGCTTCACG CTGTGGCAGGAACTGAAAGGCGAAGGCGATATCGACAACGCGCCGTGGCCGGTTGCTGA CGAAAAAGCGATGGTGGAAGACTCCACGCTGGTCGTGGTGCAGGTTAACGGTAAGGTCCG TGCCAAAATCACCGTTCCGGTGGACGCAACGGAAGAACAGGTTCGCGAACGTGCTGGCCA GGAACATCTGGTAGCAAAATATCTTGATGGCGTTACTGTACGTAAAGTGATTTACGTACCA GGTAAACTCCTCAATCTGGTCGTTGGCTAA 
ccggtaccctggaaggtgccactcccactgtcctttcctaataaaatgaggaaattg catcgcattgtctgagtaggtgtcattctattc tggggggtggggtggggcaggacagcaagggggaggattgggaagacaatagcaggcatgctggggatgcggtgggctctatgg cttctgaggcggaaagaaccagctggggctctagggggtatccccacgcgccctgtagcggcgcattaagcgcggcgggtgtggtg gttacgcgcagcgtgaccgctacacttgccagcgccctagcgcccgcactagagACGTCCCGCGGGGCGGCTCTA GACATAAAAAACAAAAAAATACCCGGAGCGGGACTTGAACCCGCACAGCGCGAACGCCGA GGGATTTAGAATCCCTTGTGTCTACCGATTCCACCATCCGGGCTATTTAATTGTTGAAGAAA GAGTATACTACATAACACATATACAATTGAAAAAGAGGCTAGCAGCTTACCGGTCGGGATG GCTGCAGgatcatttatctttcactgcggagaagtttcgaacgccgaaacatgcgcaccaactttcacttctacagcgtttgaccaa aatctttgaacagaacattgtagggtgtgaaaaatgcgcaccttaccgctagcccaagagggcactacaaaatctagagttgtact tcaaacgtacatgtaatcaccttgtatatactcgaaagaaaacatcaagtttctgtataaatatgagtgaaagcataatcatacattatcttt tcaaagactgcaggaattcgatatcaagcttCCCGGGctagagctggtaccaattccttgaattttcaaaaattcttacttttttttggat ggacgcaaagaagtttaataatcatattacatggcattaccaccatatacatatccatatctaatcttacttatatgttgtggaaatgtaaag agccccattatcttagcctaaaaaaaccttctcttggaactttcagtaatacgcttaactgctcattgctatattgaagta

aaaaaaccccggatcgaattcccta

cttcatacattttcaattaag

atgggatccgctggctccgctgctggttctg

gcgaatacgcatccaagggcgaggagctctttactggcgtagtaccaattctcgtagagctcgatggcgatgtaaatggccataagttt tccgtacgcggcgagggcgagggcgatgcaactaacggcaagctcactctcaagtttatttgtactactggcaagctcccagtaccat ggccaactctcgtaactactctgacctatggcgtacaatgttttcccgctatccagatcacatgaagcaacatgattttttaagtccgcaa tgccagagggctatgtacaagagcgcactattagcttaaggatgatggcacctataagactcgcgcagaggtaaagtttgagggcg atactctcgtaaatcgcattgagctcaagggcattgattttaaggaggatggcaatattctcggccataagctggagtataatttcaattcc cataatgtatatattaccgcagataagcaaaagaatggcattaaggcgaattttaagattcgccataatgtggaggatggctccgtaca actcgcagatcattatcaacaaaatactccaattggcgatggcccagtactcctcccagataatcattatctctccactcaatccgtgctct ccaaagatccaaatgagaagcgcgatcacatggtactcctggagtttgtaactgcagcaggcattactcatggcatggatgagctctat aagctcgagcaccaccaccaccaccactaataatagctcgagatctgataacaacagtgtagatgtaacaaaatcgactttgttccca ctgtacttttagctcgtacaaaatacaatatactttcatttctccgtaaacaacatgtttcccatgtaatatccttttctatttttcgttccgttacc aactttacacatactttatatagctattcacttctatacactaaaaaactaagacaattttaattttgctgcctgccatatttcaatttgttataaa ttcctataatttatcctattagtagctaaaaaaagatgaatgtgaatcgaatcctaagagaattgagctccaattcggtcgacctcgaggg 
ggggcccggtacccaattcgccctatagtgagtcgtattacgcgcgctcactggccgtcgttttacaacgtcgtgactgggaaaaccct ggcgttacccaacttaatcgccttgcagcacatcccctttcgccagctggcgtaatagcgaagaggcccgcaccgatcgcccttccc aacagttgcgcagcctgaatggcgaatggcgcgacgcgcctgtagcggcgcattaagcgcggcgggtgtggtggttacgcgcag cgtgaccgctacacttgccagcgcctagcgcccgctcctttcgctttcttcccttcctttctcgccacgttcgccggctttccccgtcaagct ctaaatcgggggctccctttagggttccgatttagtgctttacggcacctcgaccccaaaaaacttgattagggtgatggttcacgtagtg ggccatcgccctgatagacggttttcgcccttgacgttggagtccacgttcttaatagtggactcttgttccaaactggaacaacactca accctatctcggtctattcttttgatttataagggattttgccgatttcggcctattggttaaaaaatgagctgatttaacaaaaatttaacgcg aattttaacaaaatattaacgtttacaatttcctgatgcggtatttctccttacgcatctgtgcggtatttcacaccgcatagggtaataactg atataattaaattgaagctctaatttgtgagtttagtatacatgcatttacttataatacagtttttagttttgctggccgcatcttctcaaatatg cttcccagcctgcttttctgtaacgttcaccctctaccttagcatcccttcccttgcaaatagtcctcttccaacaataataatgtcagatcct gtagagaccacatcatccacggttctatactgttgacccaatgcgtctcccttgtcatctaaacccacaccgggtgtcataatcaaccaa tcgtaaccttcatctcttccacccatgtctctttgagcaataaagccgataacaaaatctttgtcgetcttcgcaatgtcaacagtacctta gtatattctccagtagatagggagcccttgcatgacaattctgctaacatcaaaaggcctctaggttcctttgttacttcttctgccgcctgctt caaaccgctaacaatacctgggcccaccacaccgtgtgcattcgtaatgtctgcccattctgctattctgtatacacccgcagagtactg caatttgactgtattaccaatgtcagcaaatttctgtcttcgaagagtaaaaaattgtacttggcggataatgcctttagcggcttaactgtg ccctccatggaaaaatcagtcaagatatccacatgtgttttagtaaacaaattttgggacctaatgcttcaactaactccagtaattccttg gtggtacgaacatccaatgaagcacacaagtttgtttgcttttcgtgcatgatattaaatagcttggcagcaacaggactaggatgagta gcagcacgttccttatatgtagctttcgacatgatttatcttcgtttcctgcaggttttgttctgtgcagttgggttaagaatactgggcaatttc atgtttcttcaacactacatatgcgtatatataccaatctaagtctgtgctccttccttcgttcttccttctgttcggagattaccgaatcaaaaa aattcaaagaaaccgaaatcaaaaaaaagaataaaaaaaaaatgatgaattgaattgaaaagctgtggtatggtgcactctcagt acaatctgctctgatgccgcatagttaagccagccccgacacccgccaacacccgctgacgcgccctgacgggcttgtctgctcccg gcatccgcttacagacaagctgtgaccgtctccgggagctgcatgtgtcagaggtttcaccgtcatcaccgaaacgcgcga

\section{pRS416-BXG-LeuOmeRS}

Note: original restriction enzyme sites Xbal and Sacll were eliminated at 3' and 5' ends intentionally due to presence of both restriction sites in LeuOmeRS-tRNAcuA ${ }^{\text {Leu }}$ insert.

\section{GPD Promoter, LeuOmeRS, linker, TAG codon, GFP}

gacgaaagggcctcgtgatacgcctatttttataggttaatgtcatgataataatggtttcttaggacggatcgcttgcctgtaacttacacg cgcctcgtatctttaatgatggaataatttgggaatttactctgtgtttatttattttatgttttgtatttggattttagaaagtaaataaagaaggt agaagagttacggaatgaagaaaaaaaaataaacaaaggtttaaaaaattcaacaaaaagcgtactttacatatatatttattagac aagaaaagcagattaaatagatatacattcgattaacgataagtaaaatgtaaaatcacaggatttcgtgtgtggtcttctacacagac aagatgaaacaattcggcattaatacctgagagcaggaagagcaagataaaaggtagtatttgttggcgatcccctagagtctttac atcttcggaaaacaaaaactatttttctttaatttcttttttactttctatttttaatttatatatttatattaaaaaatttaaattataattattttatag cacgtgatgaaaaggacccaggtggcacttttcggggaaatgtgcgcggaacccctatttgtttattttctaaatacattcaaatatgtat ccgctcatgagacaataaccctgataaatgcttcaataatattgaaaaaggaagagtatgagtattcaacatttccgtgtcgcccttattc cctttttgcggcatttgccttcctgttttgctcacccagaaacgctggtgaaagtaaaagatgctgaagatcagttgggtgcacgagtg ggttacatcgaactggatctcaacagcggtaagatccttgagagtttcgccccgaagaacgttttccaatgatgagcacttttaaagttct gctatgtggcgcggtattatcccgtattgacgccgggcaagagcaactcggtcgccgcatacactattctcagaatgacttggttgagta ctcaccagtcacagaaaagcatcttacggatggcatgacagtaagagaattatgcagtgctgccataaccatgagtgataacactgc ggccaacttacttctgacaacgatcggaggaccgaaggagctaaccgctttttgcacaacatgggggatcatgtaactcgccttgatc gttgggaaccggagctgaatgaagccataccaaacgacgagcgtgacaccacgatgcctgtagcaatggcaacaacgttgcgca 
aactattaactggcgaactacttactctagcttcccggcaacaattaatagactggatggaggcggataaagttgcaggaccacttctg cgctcggcccttccggctggctggtttattgctgataaatctggagccggtgagcgtgggtctcgcggtatcattgcagcactggggcca gatggtaagccctcccgtatcgtagttatctacacgacggggagtcaggcaactatggatgaacgaaatagacagatcgctgagata ggtgcctcactgattaagcattggtaactgtcagaccaagtttactcatatatactttagattgatttaaaacttcattttaatttaaaaggatc taggtgaagatccttttgataatctcatgaccaaaatcccttaacgtgagttttcgttccactgagcgtcagaccccgtagaaaagatca aaggatcttcttgagatccttttttctgcgcgtaatctgctgcttgcaaacaaaaaaaccaccgctaccagcggtggtttgtttgccggatc aagagctaccaactcttttccgaaggtaactggcttcagcagagcgcagataccaaatactgtccttctagtgtagccgtagttaggcc accacttcaagaactctgtagcaccgcctacatacctcgctctgctaatcctgttaccagtggctgctgccagtggcgataagtcgtgtct taccgggttggactcaagacgatagttaccggataaggcgcagcggtcgggctgaacggggggttcgtgcacacagccagcttgg agcgaacgacctacaccgaactgagatacctacagcgtgagctatgagaaagcgccacgcttcccgaagggagaaaggcggac aggtatccggtaagcggcagggtcggaacaggagagcgcacgagggagcttccagggggaaacgcctggtatcttatagtcctgt cgggtttcgccacctctgacttgagcgtcgattttgtgatgctcgtcaggggggcggagcctatggaaaaacgccagcaacgcggcc ttttacggttcctggccttttgctggccttttgctcacatgttctttcctgcgttatccctgattctgtggataaccgtattaccgcctttgagtga gctgataccgctcgccgcagccgaacgaccgagcgcagcgagtcagtgagcgaggaagcggaagagcgcccaatacgcaaac cgcctctccccgcgcgttggccgattcattaatgcagctggcacgacaggtttcccgactggaaagcgggcagtgagcgcaacgca attaatgtgagttacctcactcattaggcaccccaggctttacactttatgcttccggctcctatgttgtgtggaattgtgagcggataacaat ttcacacaggaaacagctatgaccatgattacgccaagcgcgcaattaaccctcactaaagggaacaaaagctggagctccaccg cCCTAGGgagctcagtttatcattatcaatactcgccatttcaaagaatacgtaaataattaatagtagtgattttcctaactttatttagt caaaaaattagccttttaattctgctgtaacccgtacatgcccaaaatagggggcgggttacacagaatatataacatcgtaggtgtctg ggtgaacagtttattcctggcatccactaaatataatggagcccgctttttaagctggcatccagaaaaaaaaagaatcccagcacca aaatattgttttcttcaccaaccatcagttcataggtccattctcttagcgcaactacagagaacaggggcacaaacaggcaaaaaac gggcacaacctcaatggagtgatgcaacctgcctggagtaaatgatgacacaaggcaattgacccacgcatgtatctatctcattttctt acaccttctattaccttctgctctctctgatttggaaaaagctgaaaaaaaaggttgaaaccagttccctgaaattattcccctacttgacta ataagtatataaagacggtaggtattgattgtaattctgtaaatctatttcttaaacttcttaaattctacttttatagttagtctttttttagttttaa aacaccagaacttagtttcgacggattctagaactagtatggagatttCCATGGAAGAGCAATACCGCCCGGAAGA GATAGAATCCAAAGTACAGCTTCATTGGGATGAGAAGCGCACATTTGAAGTAACCGAAGAC GAGAGCAAAGAGAAGTATTACTGCCTGTCTTTGGAACCCTATCCTTCTGGTCGACTACACA TGGGCCACGTACGTAACTACACCATCGGTGACGTGATCGCCCGCTACCAGCGTATGCTGG GCAAAAACGTCCTGCAGCCGATCGGCTGGGACGCGTTTGGTCTGCCTGCGGAAGGCGCG GCGGTGAAAAACAACACCGCTCCGGCACCGTGGACGTACGACAACATCGCGTATATGAAA AACCAGCTCAAAATGCTGGGCTTTGGTTATGACTGGAGCCGCGAGCTGGCAACCTGTACG CCGGAATACTACCGTTGGGAACAGAAATTCTTCACCGAGCTGTATAAAAAAGGCCTGGTAT ATAAGAAGACTTCTGCGGTCAACTGGTGCCCGAACGACCAGACCGTACTGGCGAACGAAC AAGTTATCGACGGCTGCTGCTGGCGCTGCGATACCAAAGTTGAACGTAAAGAGATCCCGC AGTGGTTTATCAAAATCACTGCTTACGCTGACGAGCTGCTCAACGATCTGGATAAACTGGA TCACTGGCCAGACACCGTTAAAACCATGCAGCGTAACTGGATCGGTCGTTCCGAAGGCGT GGAGATCACCTTCAACGTTAACGACTATGACAACACGCTGACCGTTTACACTACCCGCCCG GACGCTTTTATGGGTTGTACCTACCTGACGGTAGCTGCGGGTCATCCGCTGGCGCAGAAA GCGGCGGAAAATAATCCTGAACTGGCGGCCTTTATTGACGAATGCCGTAACACCAAAGTTG CCGAAGCTGAAATGGCGACGATGGAGAAAAAAGGCGTCGATACTGGCTTTAAAGCGGTTC ACCCATTAACGGGCGAAGAAATTCCCGTTTGGGCAGCAAACTTCGTATTGATGGAGTACGG CACGGGCGCAGTTATGGCGGTACCGGGGCACGACCAGCGCGACTACGAGTTTGCCTCTA AATACGGCCTGAACATCAAACCGGTTATCCTGGCAGCTGACGGCTCTGAGCCAGATCTTTC TCAGCAAGCCCTGACTGAAAAAGGCGTGCTGTTCAACTCTGGCGAGTTCAACGGTCTTGA 
CCATGAAGCGGCCTTCAACGCCATCGCCGATAAACTGACTGCGATGGGCGTTGGCGAGC GTAAATTGAACTACCGCCTGCGCGACTGGGGTGTTTCCCGTCAGCGTTACTGGGGCGCGC CGATTCCGATGGTGACGCTGGAAGACGGTACCGTAATGCCGACCCCGGACGACCAGCTG CCGGTGATCCTGCCGGAGGATGTGGTAATGGACGGCATTACCAGCCCGATTAAAGCAGAT CCGGAGTGGGCGAAAACTACCGTTAACGGTATGCCAGCACTGCGTGAAACCGACACTTTC GACACCTTTATGGAGTCCTCCTGGAGATATGCGCGCTACACTTGCCCGCAGTACAAAGAA GGTATGCTGGATTCCGAAGCGGCTAACTACTGGCTGCCGGTGGATATCGCTATTGGTGGT ATTGAACACGCCATTATGGGTCTGCTCTACTTCCGCTTCTTCCACAAACTGATGCGTGATG CAGGCATGGTGAACTCTGACGAACCAGCGAAACAGTTGCTGTGTCAGGGTATGGTGCTGG CAGATGCCTTCTACTATGTTGGCGAAAACGGCGAACGTAACTGGGTTTCCCCGGTTGATGC TATCGTTGAACGTGACGAGAAAGGCCGTATCGTGAAAGCGAAAGATGCGGCAGGCCATGA ACTGGTTTATACCGGCATGAGCAAAATGTCCAAGTCGAAGAACAACGGTATCGACCCGCA GGTGATGGTTGAACGTTACGGCGCGGACACCGTTCGTCTGTTTATGATGTTTGCTTCTCCG GCTGATATGACTCTCGAATGGCAGGAATCCGGTGTGGAAGGGGCTAACCGCTTCCTGAAA CGTGTCTGGAAACTGGTTTACGAGCACACAGCAAAAGGTGATGTTGCGGCACTGAACGTT GATGCGCTGACTGAAAATCAGAAAGCGCTGCGTCGCGATGTGCATAAAACGATCGCTAAA GTGACCGATGATATCGGCCGTCGTCAGACCTTCAACACCGCAATTGCGGCGATTATGGAG CTGATGAACAAACTGGCGAAAGCACCAACCGATGGCGAGCAGGATCGCGCTCTGATGCAG GAAGCACTGCTGGCCGTTGTCCGTATGCTTAACCCGTTCACCCCGCACATCTGCTTCACG CTGTGGCAGGAACTGAAAGGCGAAGGCGATATCGACAACGCGCCGTGGCCGGTTGCTGA CGAAAAAGCGATGGTGGAAGACTCCACGCTGGTCGTGGTGCAGGTTAACGGTAAGGTCCG TGCCAAAATCACCGTTCCGGTGGACGCAACGGAAGAACAGGTTCGCGAACGTGCTGGCCA GGAACATCTGGTAGCAAAATATCTTGATGGCGTTACTGTACGTAAAGTGATTTACGTACCA GGTAAACTCCTCAATCTGGTCGTTGGCTAA

ccggtaccctggaaggtgccactcccactgtcctttcctaataaaatgaggaaattgcatcgcattgtctgagtaggtgtcattctattc tggggggtggggtggggcaggacagcaagggggaggattgggaagacaatagcaggcatgctggggatgcggtgggctctatgg cttctgaggcggaaagaaccagctggggctctagggggtatccccacgcgccctgtagcggcgcattaagcgcggcgggtgtggtg gttacgcgcagcgtgaccgctacacttgccagcgccctagcgcccgcactagagACGTCCCGCGGGGCGGCTCTA GACATAAAAAACAAAAAAATACCCGGAGCGGGACTTGAACCCGCACAGCGCGAACGCCGA GGGATTTAGAATCCCTTGTGTCTACCGATTCCACCATCCGGGCTATTTAATTGTTGAAGAAA GAGTATACTACATAACACATATACAATTGAAAAAGAGGCTAGCAGCTTACCGGTCGGGATG GCTGCAGgatcatttatctttcactgcggagaagtttcgaacgccgaaacatgcgcaccaactttcacttctacagcgtttgaccaa aatctttgaacagaacattgtagggtgtgaaaaaatgcgcacctttaccgctagcccaagagggcactacaaaatctagagttgtact tcaaacgtacatgtaatcaccttgtatatactcgaaagaaaacatcaagtttctgtataaatatgagtgaaagcataatcatacattatcttt tcaaagactgcaggaattcgatatcaagcttCCCGGGctagagctggtaccaattccttgaattttcaaaaattcttacttttttttggat ggacgcaaagaagtttaataatcatattacatggcattaccaccatatacatatccatatctaatcttacttatatgttgtggaaatgtaaag agccccattatcttagcctaaaaaaaccttctcttggaactttcagtaatacgcttaactgctcattgctatattgaagta

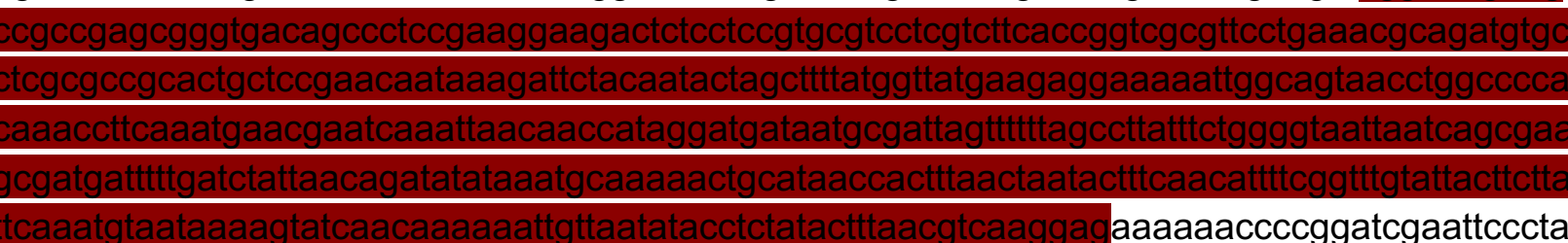


cttcatacattttcaattaag

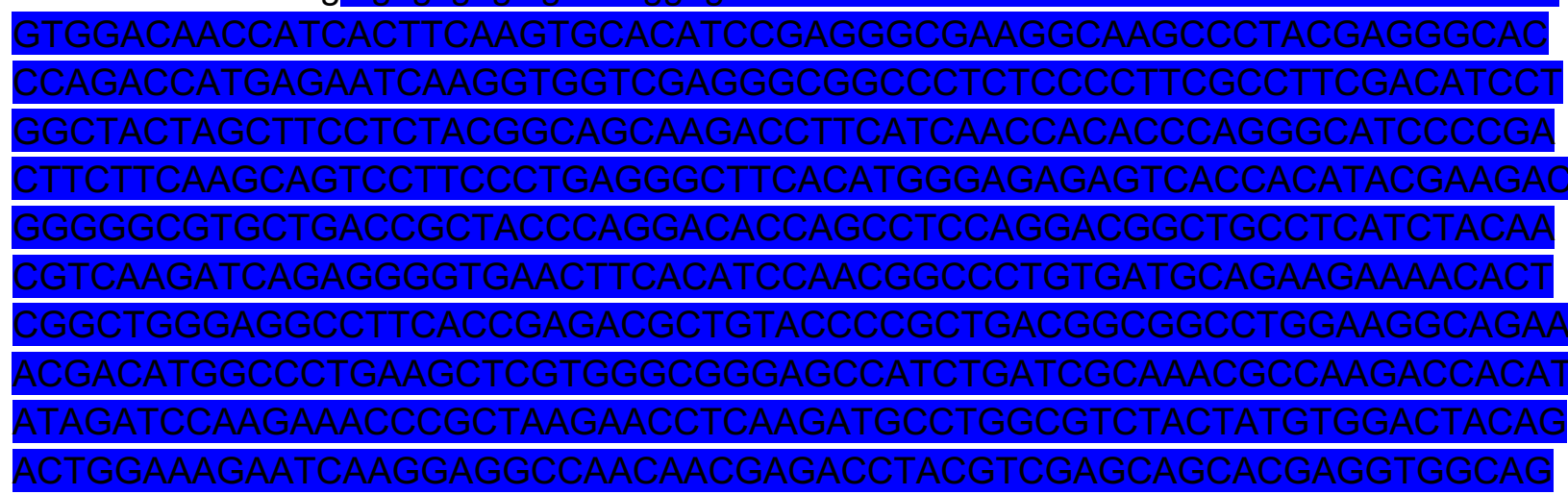

atgggatccgctggctccgctgctggttctg

gcgaaTAGgcatccaagggcgaggagctcttactggcgtagtaccaattctcgtagagctcgatggcgatgtaaatggccataagt tttccgtacgcggcgagggcgagggcgatgcaactaacggcaagctcactctcaagtttatttgtactactggcaagctcccagtacca tggccaactctcgtaactactctgacctatggcgtacaatgttttcccgctatccagatcacatgaagcaacatgattttttaagtccgca atgccagagggctatgtacaagagcgcactattagctttaaggatgatggcacctataagactcgcgcagaggtaaagtttgagggc gatactctcgtaaatcgcattgagctcaagggcattgattttaaggaggatggcaatattctcggccataagctggagtataatttcaattc ccataatgtatatattaccgcagataagcaaaagaatggcattaaggcgaatttaagattcgccataatgtggaggatggctccgtac aactcgcagatcattatcaacaaaatactccaattggcgatggcccagtactcctcccagataatcattatctctccactcaatccgtgct ctccaaagatccaaatgagaagcgcgatcacatggtactcctggagttgtaactgcagcaggcattactcatggcatggatgagctct ataagctcgagcaccaccaccaccaccactaataatagctcgagatctgataacaacagtgtagatgtaacaaaatcgactttgttcc cactgtacttttagctcgtacaaaatacaatatactttcatttctccgtaaacaacatgtttcccatgtaatatcctttctattttcgttccgtta ccaactttacacatactttatatagctattcacttctatacactaaaaaactaagacaattttaattttgctgcctgccatatttcaatttgttata aattcctataatttatcctattagtagctaaaaaaagatgaatgtgaatcgaatcctaagagaattgagctccaattcggtcgacctcgag ggggggcccggtacccaattcgccctatagtgagtcgtattacgcgcgctcactggccgtcgtttacaacgtcgtgactgggaaaacc ctggcgttacccaacttaatcgccttgcagcacatcccctttcgccagctggcgtaatagcgaagaggcccgcaccgatcgcccttcc caacagttgcgcagcctgaatggcgaatggcgcgacgcgcctgtagcggcgcattaagcgcggcgggtgtggtggttacgcgca gcgtgaccgctacacttgccagcgccctagcgcccgctcctttcgctttcttccttcctttctcgccacgttcgccggctttccccgtcaag ctctaaatcgggggctccctttagggttccgatttagtgctttacggcacctcgaccccaaaaaacttgattagggtgatggttcacgtagt gggccatcgccctgatagacggttttcgcccttgacgttggagtccacgttctttaatagtggactcttgttccaaactggaacaacactc aaccctatctcggtctattctttgatttataagggatttgccgatttcggcctattggttaaaaaatgagctgatttaacaaaaatttaacgc gaattttaacaaaatattaacgtttacaatttcctgatgcggtattttctccttacgcatctgtgcggtatttcacaccgcatagggtaataact gatataattaaattgaagctctaatttgtgagtttagtatacatg catttacttataatacagtttttagtttgctggccgcatcttctcaaatat gcttcccagcctgcttttctgtaacgttcaccctctaccttagcatcccttccctttgcaaatagtcctcttccaacaataataatgtcagatcc tgtagagaccacatcatccacggttctatactgttgacccaatgcgtctcccttgtcatctaaacccacaccgggtgtcataatcaacca atcgtaaccttcatctcttccacccatgtctctttgagcaataaagccgataacaaaatctttgtcgctcttcgcaatgtcaacagtaccctt agtatattctccagtagatagggagcccttgcatgacaattctgctaacatcaaaaggcctctaggttcctttgttacttcttctgccgcctgc ttcaaaccgctaacaatacctgggcccaccacaccgtgtgcattcgtaatgtctgcccattctgctattctgtatacacccgcagagtact gcaatttgactgtattaccaatgtcagcaaatttctgtcttcgaagagtaaaaaattgtacttggcggataatgcctttagcggcttaactgt gccctccatggaaaaatcagtcaagatatccacatgtgttttagtaaacaaatttgggacctaatgcttcaactaactccagtaattcctt ggtggtacgaacatccaatgaagcacacaagtttgtttgcttttcgtgcatgatattaaatagcttggcagcaacaggactaggatgagt agcagcacgttccttatatgtagcttcgacatgatttatcttcgtttcctgcaggttttgttctgtgcagttgggttaagaatactgggcaattt catgtttcttcaacactacatatgcgtatatataccaatctaagtctgtgctccttccttcgttcttccttctgttcggagattaccgaatcaaaa aaattcaaagaaaccgaaatcaaaaaaaagaataaaaaaaaaatgatgaattgaattgaaaagctgtggtatggtgcactctcag 
tacaatctgctctgatgccgcatagttaagccagccccgacacccgccaacacccgctgacgcgccctgacgggcttgtctgctcccg gcatccgcttacagacaagctgtgaccgtctccgggagctgcatgtgtcagaggtttcaccgtcatcaccgaaacgcgcga

\section{pRS416-BXG-altTAG-LeuOmeRS}

Note: original restriction enzyme sites Xbal and Sacll were eliminated at 3' and 5' ends intentionally due to presence of both restriction sites in LeuOmeRS-tRNAcuA ${ }^{\text {Leu }}$ insert.

\section{GPD Promoter, LeuOmeRS, CYC1 terminator, tRNACUA ${ }^{\text {Leu }, ~ S N R 52 ~ p r o m o t e r, ~ G A L 1, ~ B F P, ~}$ linker, TAG codon, GFP}

gacgaaagggcctcgtgatacgcctattttataggttaatgtcatgataataatggtttcttaggacggatcgcttgcctgtaacttacacg cgcctcgtatcttttaatgatggaataatttgggaatttactctgtgtttatttattttatgtttgtatttggattttagaaagtaaataaagaaggt agaagagttacggaatgaagaaaaaaaaataaacaaaggtttaaaaaatttcaacaaaaagcgtactttacatatatatttattagac aagaaaagcagattaaatagatatacattcgattaacgataagtaaaatgtaaaatcacaggatttcgtgtgtggtcttctacacagac aagatgaaacaattcggcattaatacctgagagcaggaagagcaagataaaaggtagtatttgttggcgatcccctagagtctttac atcttcggaaaacaaaaactatttttctttaatttcttttttactttctatttttaatttatatatttatattaaaaaatttaaattataattattttatag cacgtgatgaaaaggacccaggtggcacttttcggggaaatgtgcgcggaacccctatttgtttattttctaaatacattcaaatatgtat ccgctcatgagacaataaccctgataaatgcttcaataatattgaaaaaggaagagtatgagtattcaacatttccgtgtcgcccttattc cctttttgcggcatttgccttcctgttttgctcacccagaaacgctggtgaaagtaaaagatgctgaagatcagttgggtgcacgagtg ggttacatcgaactggatctcaacagcggtaagatccttgagagtttcgccccgaagaacgtttccaatgatgagcacttttaaagttct gctatgtggcgcggtattatcccgtattgacgccgggcaagagcaactcggtcgccgcatacactattctcagaatgacttggttgagta ctcaccagtcacagaaaagcatcttacggatggcatgacagtaagagaattatgcagtgctgccataaccatgagtgataacactgc ggccaacttacttctgacaacgatcggaggaccgaaggagctaaccgctttttgcacaacatgggggatcatgtaactcgccttgatc gttgggaaccggagctgaatgaagccataccaaacgacgagcgtgacaccacgatgcctgtagcaatggcaacaacgttgcgca aactattaactggcgaactacttactctagcttcccggcaacaattaatagactggatggaggcggataaagttgcaggaccacttctg cgctcggcccttccggctggctggtttattgctgataaatctggagccggtgagcgtgggtctcgcggtatcattgcagcactggggcca gatggtaagccctcccgtatcgtagttatctacacgacggggagtcaggcaactatggatgaacgaaatagacagatcgctgagata ggtgcctcactgattaagcattggtaactgtcagaccaagtttactcatatatactttagattgatttaaaacttcattttaatttaaaaggatc taggtgaagatccttttgataatctcatgaccaaaatcccttaacgtgagttttcgttccactgagcgtcagaccccgtagaaaagatca aaggatcttcttgagatccttttttctgcgcgtaatctgctgcttgcaaacaaaaaaaccaccgctaccagcggtggtttgtttgccggatc aagagctaccaactcttttccgaaggtaactggcttcag cagagcgcagataccaaatactgtccttctagtgtagccgtagttaggcc accacttcaagaactctgtagcaccgcctacatacctcgctctgctaatcctgttaccagtggctgctgccagtggcgataagtcgtgtct taccgggttggactcaagacgatagttaccggataaggcgcagcggtcgggctgaacggggggttcgtgcacacagcccagcttgg agcgaacgacctacaccgaactgagatacctacagcgtgagctatgagaaagcgccacgcttcccgaagggagaaaggcggac aggtatccggtaagcggcagggtcggaacaggagagcgcacgagggagcttccagggggaaacgcctggtatcttatagtcctgt cgggtttcgccacctctgacttgagcgtcgattttgtgatgctcgtcaggggggcggagcctatggaaaaacgccagcaacgcggcc ttttacggttcctggccttttgctggccttttgctcacatgttcttcctgcgttatccctgattctgtggataaccgtattaccgcctttgagtga gctgataccgctcgccgcagccgaacgaccgagcgcagcgagtcagtgagcgaggaagcggaagagcgcccaatacgcaaac cgcctctccccgcgcgttggccgattcattaatgcagctggcacgacaggtttcccgactggaaagcgggcagtgagcgcaacgca attaatgtgagttacctcactcattaggcacccaggctttacactttatgcttccggctcctatgttgtgtggaattgtgagcggataacaat ttcacacaggaaacagctatgaccatgattacgccaagcgcgcaattaaccctcactaaagggaacaaaagctggagctccaccg cCCTAGGgagctcagtttatcattatcaatactcgccatttcaaagaatacgtaaataattaatagtagtgattttcctaactttatttagt caaaaaattagccttttaattctgctgtaacccgtacatgcccaaaatagggggcgggttacacagaatatataacatcgtaggtgtctg ggtgaacagtttattcctggcatccactaaatataatggagcccgcttttaagctggcatccagaaaaaaaaagaatcccagcacca 
aaatattgtttcttcaccaaccatcagttcataggtccattctcttagcgcaactacagagaacaggggcacaaacaggcaaaaaac gggcacaacctcaatggagtgatgcaacctgcctggagtaaatgatgacacaaggcaattgacccacgcatgtatctatctcattttctt acaccttctattaccttctgctctctctgatttggaaaaagctgaaaaaaaaggttgaaaccagttccctgaaattattcccctacttgacta ataagtatataaagacggtaggtattgattgtaattctgtaaatctatttcttaaacttcttaaattctacttttatagttagtctttttttagttttaa aacaccagaacttagtttcgacggattctagaactagtatggagatttCCATGGAAGAGCAATACCGCCCGGAAGA GATAGAATCCAAAGTACAGCTTCATTGGGATGAGAAGCGCACATTTGAAGTAACCGAAGAC GAGAGCAAAGAGAAGTATTACTGCCTGTCTTTGGAACCCTATCCTTCTGGTCGACTACACA TGGGCCACGTACGTAACTACACCATCGGTGACGTGATCGCCCGCTACCAGCGTATGCTGG GCAAAAACGTCCTGCAGCCGATCGGCTGGGACGCGTTTGGTCTGCCTGCGGAAGGCGCG GCGGTGAAAAACAACACCGCTCCGGCACCGTGGACGTACGACAACATCGCGTATATGAAA AACCAGCTCAAAATGCTGGGCTTTGGTTATGACTGGAGCCGCGAGCTGGCAACCTGTACG CCGGAATACTACCGTTGGGAACAGAAATTCTTCACCGAGCTGTATAAAAAAGGCCTGGTAT ATAAGAAGACTTCTGCGGTCAACTGGTGCCCGAACGACCAGACCGTACTGGCGAACGAAC AAGTTATCGACGGCTGCTGCTGGCGCTGCGATACCAAAGTTGAACGTAAAGAGATCCCGC AGTGGTTTATCAAAATCACTGCTTACGCTGACGAGCTGCTCAACGATCTGGATAAACTGGA TCACTGGCCAGACACCGTTAAAACCATGCAGCGTAACTGGATCGGTCGTTCCGAAGGCGT GGAGATCACCTTCAACGTTAACGACTATGACAACACGCTGACCGTTTACACTACCCGCCCG GACGCTTTTATGGGTTGTACCTACCTGACGGTAGCTGCGGGTCATCCGCTGGCGCAGAAA GCGGCGGAAAATAATCCTGAACTGGCGGCCTTTATTGACGAATGCCGTAACACCAAAGTTG CCGAAGCTGAAATGGCGACGATGGAGAAAAAAGGCGTCGATACTGGCTTTAAAGCGGTTC ACCCATTAACGGGCGAAGAAATTCCCGTTTGGGCAGCAAACTTCGTATTGATGGAGTACGG CACGGGCGCAGTTATGGCGGTACCGGGGCACGACCAGCGCGACTACGAGTTTGCCTCTA AATACGGCCTGAACATCAAACCGGTTATCCTGGCAGCTGACGGCTCTGAGCCAGATCTTTC TCAGCAAGCCCTGACTGAAAAAGGCGTGCTGTTCAACTCTGGCGAGTTCAACGGTCTTGA CCATGAAGCGGCCTTCAACGCCATCGCCGATAAACTGACTGCGATGGGCGTTGGCGAGC GTAAATTGAACTACCGCCTGCGCGACTGGGGTGTTTCCCGTCAGCGTTACTGGGGCGCGC CGATTCCGATGGTGACGCTGGAAGACGGTACCGTAATGCCGACCCCGGACGACCAGCTG CCGGTGATCCTGCCGGAGGATGTGGTAATGGACGGCATTACCAGCCCGATTAAAGCAGAT CCGGAGTGGGCGAAAACTACCGTTAACGGTATGCCAGCACTGCGTGAAACCGACACTTTC GACACCTTTATGGAGTCCTCCTGGAGATATGCGCGCTACACTTGCCCGCAGTACAAAGAA GGTATGCTGGATTCCGAAGCGGCTAACTACTGGCTGCCGGTGGATATCGCTATTGGTGGT ATTGAACACGCCATTATGGGTCTGCTCTACTTCCGCTTCTTCCACAAACTGATGCGTGATG CAGGCATGGTGAACTCTGACGAACCAGCGAAACAGTTGCTGTGTCAGGGTATGGTGCTGG CAGATGCCTTCTACTATGTTGGCGAAAACGGCGAACGTAACTGGGTTTCCCCGGTTGATGC TATCGTTGAACGTGACGAGAAAGGCCGTATCGTGAAAGCGAAAGATGCGGCAGGCCATGA ACTGGTTTATACCGGCATGAGCAAAATGTCCAAGTCGAAGAACAACGGTATCGACCCGCA GGTGATGGTTGAACGTTACGGCGCGGACACCGTTCGTCTGTTTATGATGTTTGCTTCTCCG GCTGATATGACTCTCGAATGGCAGGAATCCGGTGTGGAAGGGGCTAACCGCTTCCTGAAA CGTGTCTGGAAACTGGTTTACGAGCACACAGCAAAAGGTGATGTTGCGGCACTGAACGTT GATGCGCTGACTGAAAATCAGAAAGCGCTGCGTCGCGATGTGCATAAAACGATCGCTAAA GTGACCGATGATATCGGCCGTCGTCAGACCTTCAACACCGCAATTGCGGCGATTATGGAG CTGATGAACAAACTGGCGAAAGCACCAACCGATGGCGAGCAGGATCGCGCTCTGATGCAG GAAGCACTGCTGGCCGTTGTCCGTATGCTTAACCCGTTCACCCCGCACATCTGCTTCACG CTGTGGCAGGAACTGAAAGGCGAAGGCGATATCGACAACGCGCCGTGGCCGGTTGCTGA 
CGAAAAAGCGATGGTGGAAGACTCCACGCTGGTCGTGGTGCAGGTTAACGGTAAGGTCCG TGCCAAAATCACCGTTCCGGTGGACGCAACGGAAGAACAGGTTCGCGAACGTGCTGGCCA GGAACATCTGGTAGCAAAATATCTTGATGGCGTTACTGTACGTAAAGTGATTTACGTACCA GGTAAACTCCTCAATCTGGTCGTTGGCTAACA

ccggtacccctggaaggtgccactcccactgtccttcctaataaaatgaggaaattgcatcgcattgtctgagtaggtgtcattctattc tggggggtggggtggggcaggacagcaagggggaggattgggaagacaatagcaggcatgctggggatgcggtgggctctatgg cttctgaggcggaaagaaccagctggggctctagggggtatcccacgcgccctgtagcggcgcattaagcgcggcgggtgtggtg gttacgcgcagcgtgaccgctacacttgccagcgccctagcgcccgcactagagACGTCCCGCGGGGCGGCTCTA GACATAAAAAACAAAAAAATACCCGGAGCGGGACTTGAACCCGCACAGCGCGAACGCCGA GGGATTTAGAATCCCTTGTGTCTACCGATTCCACCATCCGGGCTATTTAATTGTTGAAGAAA GAGTATACTACATAACACATATACAATTGAAAAAGAGGCTAGCAGCTTACCGGTCGGGATG GCTGCAGgatcatttatctttcactgcggagaagtttcgaacgccgaaacatgcgcaccaactttcacttctacagcgtttgaccaa aatctttgaacagaacattgtagggtgtgaaaaaatgcg caccttaccgctagcccaagagggcactacaaaatctagagttgtact tcaaacgtacatgtaatcaccttgtatatactcgaaagaaaacatcaagtttctgtataaatatgagtgaaagcataatcatacattatcttt tcaaagactgcaggaattcgatatcaagcttCCCGGGctagagctggtaccaattccttgaattttcaaaaattcttactttttttggat ggacgcaaagaagtttaataatcatattacatggcattaccaccatatacatatccatatctaatcttacttatatgttgtggaaatgtaaag agccccattatcttagcctaaaaaaaccttctcttggaactttcagtaatacg ttaactgctcattgctatattgaagta

cttcatacatttcaattaag

latgggaTAGgctggctccgctgctggttc tggcgaatacgcatccaagggcgaggagctctttactggcgtagtaccaattctcgtagagctcgatggcgatgtaaatggccataag ttttccgtacgcggcgagggcgagggcgatgcaactaacggcaagctcactctcaagtttatttgtactactggcaagctcccagtacc atggccaactctcgtaactactctgacctatggcgtacaatgttttcccgctatccagatcacatgaagcaacatgattttttaagtccgc aatgccagagggctatgtacaagagcgcactattagctttaaggatgatggcacctataagactcgcgcagaggtaaagtttgaggg cgatactctcgtaaatcgcattgagctcaagggcattgattttaaggaggatggcaatattctcggccataagctggagtataatttcaatt cccataatgtatatattaccgcagataagcaaaagaatggcattaaggcgaatttaagattcgccataatgtggaggatggctccgta caactcgcagatcattatcaacaaaatactccaattggcgatggcccagtactcctcccagataatcattatctctccactcaatccgtgc tctccaaagatccaaatgagaagcgcgatcacatggtactcctggagtttgtaactgcagcaggcattactcatggcatggatgagctc 
tataagctcgagcaccaccaccaccaccactaataatagctcgagatctgataacaacagtgtagatgtaacaaaatcgacttgttc ccactgtacttttag ctcgtacaaaatacaatatactttcatttctccgtaaacaacatgtttcccatgtaatatccttttctattttcgttccgtt accaactttacacatactttatatagctattcacttctatacactaaaaaactaagacaattttaatttgctgcctgccatatttcaatttgttat aaattcctataatttatcctattagtagctaaaaaaagatgaatgtgaatcgaatcctaagagaattgagctccaattcggtcgacctcga gggggggcccggtacccaattcgccctatagtgagtcgtattacgcgcgctcactggccgtcgtttacaacgtcgtgactgggaaaac cctggcgttacccaacttaatcgccttgcagcacatcccctttcgccagctggcgtaatagcgaagaggcccgcaccgatcgcccttc ccaacagttgcgcagcctgaatggcgaatggcgcgacgcgccctgtagcggcgcattaagcgcggcgggtgtggtggttacgcgc agcgtgaccgctacacttgccagcgccctagcgcccgctcctttcgctttcttcccttcctttctcgccacgttcgccggctttccccgtcaa gctctaaatcggggg ctccctttagggttccgatttagtgctttacggcacctcgaccccaaaaaacttgattagggtgatggttcacgta gtgggccatcgccctgatagacggttttcgccctttgacgttggagtccacgttctttaatagtggactcttgttccaaactggaacaaca ctcaaccctatctcggtctattctttgatttataagggattttgccgatttcggcctattggttaaaaaatgagctgatttaacaaaaatttaac gcgaattttaacaaaatattaacgtttacaatttcctgatgcggtattttctccttacgcatctgtgcggtatttcacaccgcatagggtaata actgatataattaaattgaagctctaatttgtgagtttagtatacatgcatttacttataatacagtttttagtttgctggccgcatcttctcaaa tatgcttcccagcctgcttttctgtaacgttcaccctctaccttagcatcccttccctttgcaaatagtcctcttccaacaataataatgtcagat cctgtagagaccacatcatccacggttctatactgttgacccaatgcgtctcccttgtcatctaaacccacaccgggtgtcataatcaacc aatcgtaaccttcatctcttccacccatgtctctttgagcaataaagccgataacaaaatctttgtcgctcttcgcaatgtcaacagtaccct tagtatattctccagtagatagggagcccttgcatgacaattctgctaacatcaaaaggcctctaggttcctttgttacttcttctgccgcctg cttcaaaccgctaacaatacctgggcccaccacaccgtgtgcattcgtaatgtctgcccattctgctattctgtatacacccgcagagtac tgcaatttgactgtattaccaatgtcagcaaatttctgtcttcgaagagtaaaaaattgtacttggcggataatgcctttagcggcttaactg tgccctccatggaaaaatcagtcaagatatccacatgtgttttagtaaacaaattttgggacctaatgcttcaactaactccagtaattcct tggtggtacgaacatccaatgaagcacacaagtttgtttgcttttcgtgcatgatattaaatagcttggcagcaacaggactaggatgagt agcagcacgttccttatatgtagctttcgacatgatttatcttcgtttcctgcaggttttgttctgtgcagttgggttaagaatactgggcaattt catgtttcttcaacactacatatgcgtatatataccaatctaagtctgtgctccttccttcgttcttccttctgttcggagattaccgaatcaaaa aaattcaaagaaaccgaaatcaaaaaaaagaataaaaaaaaaatgatgaattgaattgaaaagctgtggtatggtgcactctcag tacaatctgctctgatgccgcatagttaagccagccccgacacccgccaacacccgctgacgcgccctgacgggettgtctgctcccg gcatccgcttacagacaagctgtgaccgtctccgggagctgcatgtgtcagaggttttcaccgtcatcaccgaaacgcgcga

\section{pRS416-BYG-TyrOmeRS}

Note: original restriction enzyme sites Xbal and Sacll were eliminated at 3' and 5' ends intentionally due to presence of both restriction sites in TyrOmeRS-tRNA ${ }_{C U A}{ }^{T y r}$ insert.

\section{GPD Promoter, TyrOmeRS, CYC1 terminator, tRNACUA ${ }^{\text {Tyr }}$, SNR52 promoter, GAL1, BFP, linker, GFP}

gacgaaagggcctcgtgatacgcctattttataggttaatgtcatgataataatggtttcttaggacggatcgcttgcctgtaacttacacg cgcctcgtatctttaatgatggaataatttgggaatttactctgtgtttatttattttatgtttgtatttggattttagaaagtaaataaagaaggt agaagagttacggaatgaagaaaaaaaaataaacaaaggtttaaaaaatttcaacaaaaagcgtactttacatatatatttattagac aagaaaagcagattaaatagatatacattcgattaacgataagtaaaatgtaaaatcacaggattttcgtgtgtggtcttctacacagac aagatgaaacaattcggcattaatacctgagagcaggaagagcaagataaaaggtagtatttgttggcgatccccctagagtctttac atcttcggaaaacaaaaactatttttctttaatttcttttttactttctatttttaatttatatatttatattaaaaaatttaaattataattattttatag cacgtgatgaaaaggacccaggtggcactttcggggaaatgtgcgcggaaccctatttgtttattttctaaatacattcaaatatgtat ccgctcatgagacaataaccctgataaatgcttcaataatattgaaaaaggaagagtatgagtattcaacatttccgtgtcgcccttattc cctttttgcggcattttgccttcctgttttgctcacccagaaacgctggtgaaagtaaaagatgctgaagatcagttgggtgcacgagtg ggttacatcgaactggatctcaacagcggtaagatccttgagagtttcgccccgaagaacgtttccaatgatgagcacttttaaagttct 
gctatgtggcgcggtattatcccgtattgacgccgggcaagagcaactcggtcgccgcatacactattctcagaatgacttggttgagta ctcaccagtcacagaaaagcatcttacggatggcatgacagtaagagaattatgcagtgctgccataaccatgagtgataacactgc ggccaacttacttctgacaacgatcggaggaccgaaggagctaaccgctttttgcacaacatgggggatcatgtaactcgccttgatc gttgggaaccggagctgaatgaagccataccaaacgacgagcgtgacaccacgatgcctgtagcaatggcaacaacgttgcgca aactattaactggcgaactacttactctagcttcccggcaacaattaatagactggatggaggcggataaagttgcaggaccacttctg cgctcggcccttccggctggctggtttattgctgataaatctggagccggtgagcgtgggtctcgcggtatcattgcagcactggggcca gatggtaagccctcccgtatcgtagttatctacacgacggggagtcaggcaactatggatgaacgaaatagacagatcgctgagata ggtgcctcactgattaagcattggtaactgtcagaccaagtttactcatatatactttagattgatttaaaacttcattttaatttaaaaggatc taggtgaagatccttttgataatctcatgaccaaaatcccttaacgtgagttttcgttccactgagcgtcagaccccgtagaaaagatca aaggatcttcttgagatccttttttctgcgcgtaatctgctgcttgcaaacaaaaaaaccaccgctaccagcggtggtttgtttgccggatc aagagctaccaactcttttccgaaggtaactggcttcagcagagcgcagataccaaatactgtccttctagtgtagccgtagttaggcc accacttcaagaactctgtagcaccgcctacatacctcgctctgctaatcctgttaccagtggctgctgccagtggcgataagtcgtgtct taccgggttggactcaagacgatagttaccggataaggcgcagcggtcgggctgaacggggggttcgtgcacacagcccagcttgg agcgaacgacctacaccgaactgagatacctacagcgtgagctatgagaaagcgccacgcttcccgaagggagaaaggcggac aggtatccggtaagcggcagggtcggaacaggagagcgcacgagggagcttccagggggaaacgcctggtatcttatagtcctgt cgggtttcgccacctctgacttgagcgtcgattttgtgatgctcgtcaggggggcggagcctatggaaaaacgccagcaacgcggcc ttttacggttcctggccttttgctggccttttgctcacatgttctttcctgcgttatccctgattctgtggataaccgtattaccgcctttgagtga gctgataccgctcgccgcagccgaacgaccgagcgcagcgagtcagtgagcgaggaagcggaagagcgcccaatacgcaaac cgcctctccccgcgcgttggccgattcattaatgcagctggcacgacaggtttcccgactggaaagcgggcagtgagcgcaacgca attaatgtgagttacctcactcattaggcaccccaggctttacactttatgcttccggctcctatgttgtgtggaattgtgagcggataacaat ttcacacaggaaacagctatgaccatgattacgccaagcgcgcaattaaccctcactaaagggaacaaaagctggagctccaccg cCCTAGGgagctcagtttatcattatcaatactcgccatttcaaagaatacgtaaataattaatagtagtgattttcctaactttatttagt caaaaaattagccttttaattctgctgtaacccgtacatgcccaaaatagggggcgggttacacagaatatataacatcgtaggtgtctg ggtgaacagtttattcctggcatccactaaatataatggagcccgcttttaagctggcatccagaaaaaaaaagaatcccagcacca aaatattgtttcttcaccaaccatcagttcataggtccattctcttagcgcaactacagagaacaggggcacaaacaggcaaaaaac gggcacaacctcaatggagtgatgcaacctgcctggagtaaatgatgacacaaggcaattgacccacgcatgtatctatctcattttctt acaccttctattaccttctgctctctctgatttggaaaaagctgaaaaaaaaggttgaaaccagttccctgaaattattcccctacttgacta ataagtatataaagacggtaggtattgattgtaattctgtaaatctatttcttaaacttcttaaattctacttttatagttagtctttttttagttttaa aacaccagaacttagtttcgacggattctagaactagtatggagattttgatggcaagcagtaacttgattaaacaattgcaagagcgg gggctggtagcccaggtgacggacgaggaagcgttagcagagcgactggcgcaaggcccgatcgcgctcacctgcggcttcgatc ctaccgctgacagcttgcatttggggcatcttgttccattgttatgcctgaaacgcttccagcaggcgggccacaagccggttgcgctgg aggcggcgcgacgggtctgattggcgacccgagcttcaaagctgccgagcgtaagctgaacactgaagaaactgttcaggagtgg gtggacaaaatccgcaagcaggttgccccgttcctcgatttcgactgtggagaaaactctgctatcgcggcgaacaactatgactggtt cggcaatatgaatgtgctgaccttcctgcgcgatattggcaaacacttctccgttaaccagatgatcaacaaagaagcggttaagcag cgtctcaaccgtgaagatcaggggatttcgttcactgagtttcctacaacctgttgcagggttataccatggcctgtctgaacaaacagt acggtgtggtgctgcaaattggtggttctgaccagtggggtaacatcacttctggtatcgacctgacccgtcgtctgcatcagaatcagg gtttggcctgaccgttccgctgatcactaaagcagatggcaccaaatttggtaaaactgaaggcggcgcagtctggttggatccgaag aaaaccagcccgtacaaattctaccagttctggatcaacactgcggatgccgacgtttaccgcttcctgaagttcttcacctttatgagca ttgaagagatcaacgccctggaagaagaagataaaaacagcggtaaagcaccgcgcgcccagtatgtactggcggagcaggtg actcgtctggttcacggtgaagaaggtttacaggcggcaaaacgtattaccgaatgcctgttcagcggttctttgagtgcgctgagtgaa gcggacttcgaacagctggcgcaggacggcgtaccgatggttgagatggaaaagggcgcagacctgatgcaggcactggtcgatt ctgaactgcaaccttcccgtggtcaggcacgtaaaactatcgcctccaatgccatcaccattaacggtgaaaaacagtccgatcctga atacttctttaaagaagaagatcgtctgtttggtcgttttaccttactgcgtcgtggcaaaaagaattactgtctgatttgctggaaataact 
gccggtacccctggaaggtgccactcccac tgtccttcctaataaaatgaggaaattgcatcgcattgtctgagtaggtgtcattctattctggggggtggggtggggcaggacagcaa gggggaggattgggaagacaatagcaggcatgctggggatgcggtgggctctatggcttctgaggcggaaagaaccagctgggg ctctagggggtatcccacgcgcctgtagcggcgcattaagcgcggcgggtgtggtggttacgcgcagcgtgaccgctacacttgc cagcgccctagcgcccgcactagagtcgacagacataaaaaacaaaaaaatggtgggggaaggattcgaaccttcgaagtcgat gacggcagatttagagtctgctccctttggccgctcgggaaccccaccgatcatttatctttcactgcggagaagtttcgaacgccgaaa catgcgcaccaactttcacttctacagcgtttgaccaaaatctttgaacagaacattgtagggtgtgaaaaaatg gcacctttaccgct agcccaagagggcactacaaaatctagagttgtacttcaaacgtacatgtaatcaccttgtatatactcgaaagaaaacatcaagttc tgtataaatatgagtgaaagcataatcatacattatcttttcaaagactgcaggaattcgatatcaagcttCCCGGGctagagctggt accaattccttgaattttcaaaaattcttacttttttttggatggacgcaaagaagtttaataatcatattacatggcattaccaccatatacat atccatatctaatcttacttatatgttgtggaaatgtaaagagccccattatcttagcctaaaaaaaccttctcttggaactttcagtaatac gcttaactgctcattgctatattgaagta

atttctggggtaattaatcagcgaagcgatgattttgatctattaacagatatataaatgcaaaaactgcataac cactttaactaatactttcaacattttcggtttgtattacttcttattcaaatgtaataaaagtatcaacaaaaaattgttaatatacctctatactt taacgtcaaggagaaaaaaccccggatcgaattccctacttcatacattttcaattaag

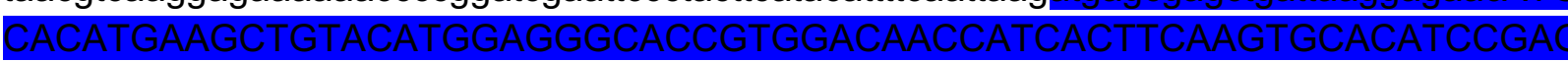

atgggatccgctggctccgctgctggttctggcgaatacgcatccaagggcgaggagctctttactggcgtagtacca attctcgtagagctcgatggcgatgtaaatggccataagtttccgtacgcggcgagggcgagggcgatgcaactaacggcaagctc actctcaagtttatttgtactactggcaagctcccagtaccatggccaactctcgtaactactctgacctatggcgtacaatgttttcccgct atccagatcacatgaagcaacatgattttttaagtccgcaatgccagagggctatgtacaagagcgcactattagctttaaggatgatg gcacctataagactcgcgcagaggtaaagtttgagggcgatactctcgtaaatcgcattgagctcaagggcattgattttaaggaggat ggcaatattctcggccataagctggagtataatttcaattcccataatgtatatattaccgcagataagcaaaagaatggcattaaggcg aattttaagattcgccataatgtggaggatggctccgtacaactcgcagatcattatcaacaaaatactccaattggcgatggcccagta ctcctcccagataatcattatctctccactcaatccgtgctctccaaagatccaaatgagaagcgcgatcacatggtactcctggagtttg taactgcagcaggcattactcatggcatggatgagctctataagctcgagcaccaccaccaccaccactaataatagctcgagatctg ataacaacagtgtagatgtaacaaaatcgactttgttcccactgtacttttagctcgtacaaaatacaatatactttcatttctccgtaaaca acatgttttcccatgtaatatccttttctattttcgttccgttaccaactttacacatactttatatagctattcacttctatacactaaaaaactaa gacaattttaattttgctgcctgccatatttcaatttgttataaattcctataatttatcctattagtagctaaaaaaagatgaatgtgaatcgaa tcctaagagaattgagctccaattcggtcgacctcgagggggggcccggtacccaattcgccctatagtgagtcgtattacgcgcgctc actggccgtcgttttacaacgtcgtgactgggaaaaccctggcgttacccaacttaatcgccttgcagcacatccccetttcgccagctg gcgtaatagcgaagaggcccgcaccgatcgcccttcccaacagttgcgcagcctgaatggcgaatggcgcgacgcgccctgtagc 
ggcgcattaagcgcggcgggtgtggtggttacgcgcagcgtgaccgctacacttgccagcgccctagcgcccgctcctttcgctttcttc ccttcctttctcgccacgttcgccggcttccccgtcaagctctaaatcgggggctccctttagggttccgatttagtgctttacggcacctcg acccaaaaaacttgattagggtgatggttcacgtagtgggccatcgccctgatagacggttttcgccctttgacgttggagtccacgtt ctttaatagtggactcttgttccaaactggaacaacactcaaccctatctcggtctattctttgatttataagggattttgccgatttcggccta ttggttaaaaaatgagctgatttaacaaaaatttaacgcgaattttaacaaaatattaacgtttacaatttcctgatgcggtattttctccttac gcatctgtgcggtatttcacaccgcatagggtaataactgatataattaaattgaagctctaatttgtgagtttagtatacatgcatttacttat aatacagtttttagttttgctggccgcatcttctcaaatatgcttcccagcctgctttctgtaacgttcaccctctaccttagcatcccttcccttt gcaaatagtcctcttccaacaataataatgtcagatcctgtagagaccacatcatccacggttctatactgttgacccaatgcgtctccctt gtcatctaaacccacaccgggtgtcataatcaaccaatcgtaaccttcatctcttccacccatgtctctttgagcaataaagccgataac aaaatcttgtcgctcttcgcaatgtcaacagtacccttagtatattctccagtagatagggagcccttgcatgacaattctgctaacatca aaaggcctctaggttcctttgttacttcttctgccgcctgcttcaaaccgctaacaatacctgggcccaccacaccgtgtgcattcgtaatgt ctgcccattctgctattctgtatacacccgcagagtactgcaatttgactgtattaccaatgtcagcaaattttctgtcttcgaagagtaaaa aattgtacttggcggataatgcctttagcggcttaactgtgccctccatggaaaaatcagtcaagatatccacatgtgttttagtaaacaa atttgggacctaatgcttcaactaactccagtaattccttggtggtacgaacatccaatgaagcacacaagtttgttgcttttcgtgcatga tattaaatagcttggcagcaacaggactaggatgagtagcagcacgttccttatatgtagctttcgacatgatttatcttcgtttcctgcagg ttttgttctgtgcagttgggttaagaatactggg caatttcatgtttcttcaacactacatatgcgtatatataccaatctaagtctgtgctcctt ccttcgttcttccttctgttcggagattaccgaatcaaaaaaatttcaaagaaaccgaaatcaaaaaaaagaataaaaaaaaaatgat gaattgaattgaaaagctgtggtatggtgcactctcagtacaatctgctctgatgccgcatagttaagccagccccgacacccgccaac acccgctgacgcgccctgacgggcttgtctgctcccggcatccgcttacagacaagctgtgaccgtctccgggagctgcatgtgtcag aggttttcaccgtcatcaccgaaacgcgcga

\section{pRS416-BXG-TyrOmeRS}

Note: original restriction enzyme sites Xbal and Sacll were eliminated at 3' and 5' ends intentionally due to presence of both restriction sites in TyrOmeRS-tRNA ${ }_{c u A}{ }^{\text {Tyr }}$ insert.

\section{GPD Promoter, TyrOmeRS, CYC1 terminator, tRNACUA ${ }^{\text {Tyr }}$, SNR52 promoter, GAL1, BFP, linker, TAG codon, GFP}

gacgaaagggcctcgtgatacgcctattttataggttaatgtcatgataataatggtttcttaggacggatcgcttgcctgtaacttacacg cgcctcgtatctttaatgatggaataatttgggaatttactctgtgtttatttattttatgtttgtatttggattttagaaagtaaataaagaaggt agaagagttacggaatgaagaaaaaaaaataaacaaaggtttaaaaaatttcaacaaaaagcgtactttacatatatatttattagac aagaaaagcagattaaatagatatacattcgattaacgataagtaaaatgtaaaatcacaggattttcgtgtgtggtcttctacacagac aagatgaaacaattcggcattaatacctgagagcaggaagagcaagataaaaggtagtatttgttggcgatcccctagagtctttac atcttcggaaaacaaaaactatttttctttaatttcttttttactttctatttttaatttatatatttatattaaaaaatttaaattataattattttatag cacgtgatgaaaaggacccaggtggcacttttcggggaaatgtgcgcggaacccctatttgtttatttttctaaatacattcaaatatgtat ccgctcatgagacaataaccctgataaatgcttcaataatattgaaaaaggaagagtatgagtattcaacatttccgtgtcgcccttattc cctttttgcggcatttgccttcctgttttgctcacccagaaacgctggtgaaagtaaaagatgctgaagatcagttgggtgcacgagtg ggttacatcgaactggatctcaacagcggtaagatccttgagagtttcgccccgaagaacgtttccaatgatgagcacttttaaagttct gctatgtggcgcggtattatcccgtattgacgccggg caagagcaactcggtcgccgcatacactattctcagaatgacttggttgagta ctcaccagtcacagaaaagcatcttacggatggcatgacagtaagagaattatgcagtgctgccataaccatgagtgataacactgc ggccaacttacttctgacaacgatcggaggaccgaaggagctaaccgctttttgcacaacatgggggatcatgtaactcgccttgatc gttgggaaccggagctgaatgaagccataccaaacgacgagcgtgacaccacgatgcctgtagcaatggcaacaacgttgcgca aactattaactggcgaactacttactctagcttcccggcaacaattaatagactggatggaggcggataaagttgcaggaccacttctg cgctcggcccttccggctggctggtttattgctgataaatctggagccggtgagcgtgggtctcgcggtatcattgcagcactggggcca 
gatggtaagccctcccgtatcgtagttatctacacgacggggagtcaggcaactatggatgaacgaaatagacagatcgctgagata ggtgcctcactgattaagcattggtaactgtcagaccaagtttactcatatatactttagattgatttaaaacttcattttaatttaaaaggatc taggtgaagatccttttgataatctcatgaccaaaatcccttaacgtgagtttcgttccactgagcgtcagaccccgtagaaaagatca aaggatcttcttgagatccttttttctgcgcgtaatctgctgcttgcaaacaaaaaaaccaccgctaccagcggtggtttgtttgccggatc aagagctaccaactcttttccgaaggtaactggcttcagcagagcgcagataccaaatactgtccttctagtgtagccgtagttaggcc accacttcaagaactctgtagcaccgcctacatacctcgctctgctaatcctgttaccagtggctgctgccagtggcgataagtcgtgtct taccgggttggactcaagacgatagttaccggataaggcgcagcggtcgggctgaacggggggttcgtgcacacagcccagcttgg agcgaacgacctacaccgaactgagatacctacagcgtgagctatgagaaagcgccacgcttcccgaagggagaaaggcggac aggtatccggtaagcggcagggtcggaacaggagagcgcacgagggagcttccagggggaaacgcctggtatctttatagtcctgt cgggtttcgccacctctgacttgagcgtcgattttgtgatgctcgtcaggggggcggagcctatggaaaaacgccagcaacgcggcc ttttacggttcctggccttttgctggccttttgctcacatgttctttcctgcgttatccctgattctgtggataaccgtattaccgcctttgagtga gctgataccgctcgccgcagccgaacgaccgagcgcagcgagtcagtgagcgaggaagcggaagagcgcccaatacgcaaac cgcctctccccgcgcgttggccgattcattaatgcagctggcacgacaggtttcccgactggaaagcgggcagtgagcgcaacgca attaatgtgagttacctcactcattaggcaccccaggctttacactttatgcttccggctcctatgttgtgtggaattgtgagcggataacaat ttcacacaggaaacagctatgaccatgattacgccaagcgcgcaattaaccctcactaaagggaacaaaagctggagctccaccg cCCTAGGgagctcagtttatcattatcaatactcgccatttcaaagaatacgtaaataattaatagtagtgattttcctaactttatttagt caaaaaattagccttttaattctgctgtaacccgtacatgcccaaaatagggggcgggttacacagaatatataacatcgtaggtgtctg ggtgaacagtttattcctggcatccactaaatataatggagcccgcttttaagctggcatccagaaaaaaaaagaatcccagcacca aaatattgtttcttcaccaaccatcagttcataggtccattctcttagcgcaactacagagaacaggggcacaaacaggcaaaaaac gggcacaacctcaatggagtgatgcaacctgcctggagtaaatgatgacacaaggcaattgacccacgcatgtatctatctcattttctt acaccttctattaccttctgctctctctgatttggaaaaagctgaaaaaaaaggttgaaaccagttccctgaaattattccctacttgacta ataagtatataaagacggtaggtattgattgtaattctgtaaatctatttcttaaacttcttaaattctacttttatagttagtctttttttagttttaa aacaccagaacttagtttcgacggattctagaactagtatggagattttgatggcaagcagtaacttgattaaacaattgcaagagcgg gggctggtagcccaggtgacggacgaggaagcgttagcagagcgactggcgcaaggcccgatcgcgctcacctgcggcttcgatc ctaccgctgacagcttgcatttggggcatcttgttccattgttatgcctgaaacgcttccagcaggcgggccacaagccggttgcgctgg aggcggcgcgacgggtctgattggcgacccgagcttcaaagctgccgagcgtaagctgaacactgaagaaactgttcaggagtgg gtggacaaaatccgcaagcaggttgccccgttcctcgatttcgactgtggagaaaactctgctatcgcggcgaacaactatgactggtt cggcaatatgaatgtgctgaccttcctgcgcgatattggcaaacacttctccgttaaccagatgatcaacaaagaagcggttaagcag cgtctcaaccgtgaagatcaggggatttcgttcactgagtttcctacaacctgttgcagggttataccatggcctgtctgaacaaacagt acggtgtggtgctgcaaattggtggttctgaccagtggggtaacatcacttctggtatcgacctgacccgtcgtctgcatcagaatcagg gtttggcctgaccgttccgctgatcactaaagcagatggcaccaaatttggtaaaactgaaggcggcgcagtctggttggatccgaag aaaaccagcccgtacaaattctaccagttctggatcaacactgcggatgccgacgtttaccgcttcctgaagttcttcaccttatgagca ttgaagagatcaacgccctggaagaagaagataaaaacagcggtaaagcaccgcgcgcccagtatgtactggcggagcaggtg actcgtctggttcacggtgaagaaggttacaggcggcaaaacgtattaccgaatgcctgttcagcggttcttgagtgcgctgagtgaa gcggacttcgaacagctggcgcaggacggcgtaccgatggttgagatggaaaagggcgcagacctgatgcaggcactggtcgatt ctgaactgcaaccttcccgtggtcaggcacgtaaaactatcgcctccaatgccatcaccattaacggtgaaaaacagtccgatcctga atacttctttaaagaagaagatcgtctgtttggtcgttttaccttactgcgtcgtggcaaaaagaattactgtctgatttgctggaaataact

gccggtacccctggaaggtgccactcccac tgtcctttcctaataaaatgaggaaattgcatcgcattgtctgagtaggtgtcattctattctggggggtggggtggggcaggacagcaa gggggaggattgggaagacaatagcaggcatgctggggatgcggtgggctctatggcttctgaggcggaaagaaccagctgggg ctctagggggtatccccacgcgccctgtagcggcgcattaagcgcggcgggtgtggtggttacgcgcagcgtgaccgctacacttgc cagcgccctagcgcccgcactagagtcgacagacataaaaaacaaaaaatggtgggggaaggattcgaaccttcgaagtcgat 
gacggcagatttagagtctgctccctttggccgctcgggaaccccaccgatcatttatcttcactgcggagaagtttcgaacgccgaaa catgcgcaccaactttcacttctacagcgtttgaccaaaatctttgaacagaacattgtagggtgtgaaaaaatgcgcacctttaccgct agcccaagagggcactacaaaatctagagttgtacttcaaacgtacatgtaatcaccttgtatatactcgaaagaaaacatcaagttc tgtataaatatgagtgaaagcataatcatacattatcttttcaaagactgcaggaattcgatatcaagcttCCCGGGctagagctggt accaattccttgaattttcaaaaattcttacttttttttggatggacgcaaagaagtttaataatcatattacatggcattaccaccatatacat atccatatctaatcttacttatatgttgtggaaatgtaaagagccccattatcttagcctaaaaaaaccttctctttggaactttcagtaatac gcttaactgctcattgctatattgaagta

atttctggggtaattaatcagcgaagcgatgattttgatctattaacagatatataaatgcaaaaactgcataac cactttaactaatactttcaacattttcggtttgtattacttcttattcaaatgtaataaaagtatcaacaaaaaattgttaatatacctctatactt taacgtcaaggagaaaaaaccccggatcgaattccctacttcatacattttcaattaag

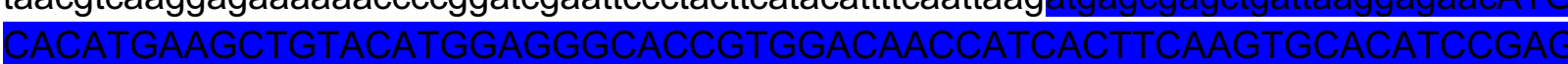

atgggatccgctggctccgctgctggttctggcgaaTAGgcatccaagggcgaggagctctttactggcgtagtacc aattctcgtagagctcgatggcgatgtaaatggccataagtttccgtacgcggcgagggcgagggcgatgcaactaacggcaagct cactctcaagtttattgtactactggcaagctcccagtaccatggccaactctcgtaactactctgacctatggcgtacaatgttttcccg ctatccagatcacatgaagcaacatgattttttaagtccgcaatgccagagggctatgtacaagagcgcactattagctttaaggatga tggcacctataagactcgcgcagaggtaaagtttgagggcgatactctcgtaaatcgcattgagctcaagggcattgattttaaggagg atggcaatattctcggccataagctggagtataatttcaattcccataatgtatatattaccgcagataagcaaaagaatggcattaagg cgaatttaagattcgccataatgtggaggatggctccgtacaactcgcagatcattatcaacaaaatactccaattggcgatggccca gtactcctcccagataatcattatctctccactcaatccgtgctctccaaagatccaaatgagaagcgcgatcacatggtactcctggag tttgtaactgcagcaggcattactcatgg catggatgagctctataagctcgagcaccaccaccaccaccactaataatagctcgagat ctgataacaacagtgtagatgtaacaaaatcgactttgttcccactgtactttagctcgtacaaaatacaatatactttcatttctccgtaa acaacatgttttcccatgtaatatccttttctattttcgttccgttaccaactttacacatactttatatagctattcacttctatacactaaaaaa ctaagacaattttaattttgctgcctgccatatttcaatttgttataaattcctataatttatcctattagtagctaaaaaaagatgaatgtgaat cgaatcctaagagaattgagctccaattcggtcgacctcgagggggggcccggtacccaattcgccctatagtgagtcgtattacgcg cgctcactggccgtcgttttacaacgtcgtgactgggaaaaccctggcgttacccaacttaatcgccttgcagcacatcccctttcgcc agctggcgtaatagcgaagaggcccgcaccgatcgcccttcccaacagttgcgcagcctgaatggcgaatggegcgacgcgccct gtagcggcgcattaagcgcggcgggtgtggtggttacgcgcagcgtgaccgctacacttgccagcgccetagcgcccgctcctttcgc tttcttcccttcctttctcgccacgttcgccggctttccccgtcaagctctaaatcgggggctccctttagggttccgatttagtgctttacggca cctcgaccccaaaaaacttgattagggtgatggttcacgtagtgggccatcgccctgatagacggttttcgccctttgacgttggagtcc acgttctttaatagtggactcttgttccaaactggaacaacactcaaccctatctcggtctattctttgatttataagggattttgccgatttcg gcctattggttaaaaaatgagctgatttaacaaaaatttaacgcgaattttaacaaaatattaacgtttacaatttcctgatgcggtattttct ccttacgcatctgtgcggtatttcacaccg catagggtaataactgatataattaaattgaagctctaatttgtgagtttagtatacatgcattt 
acttataatacagtttttagttttgctggccgcatcttctcaaatatgcttcccagcctgctttctgtaacgttcaccctctaccttagcatccct tccctttgcaaatagtcctcttccaacaataataatgtcagatcctgtagagaccacatcatccacggttctatactgttgacccaatgcgt ctcccttgtcatctaaacccacaccgggtgtcataatcaaccaatcgtaaccttcatctcttccacccatgtctctttgagcaataaagccg ataacaaaatctttgtcgctcttcgcaatgtcaacagtacccttagtatattctccagtagatagggagcccttgcatgacaattctgctaa catcaaaaggcctctaggttcctttgttacttcttctgccgcctgcttcaaaccgctaacaatacctgggcccaccacaccgtgtgcattcg taatgtctgcccattctgctattctgtatacacccgcagagtactgcaatttgactgtattaccaatgtcagcaaatttctgtcttcgaagagt aaaaaattgtacttggcggataatgcctttagcggcttaactgtgccctccatggaaaaatcagtcaagatatccacatgtgttttagtaa acaaatttgggacctaatgcttcaactaactccagtaattccttggtggtacgaacatccaatgaagcacacaagtttgtttgcttttcgtg catgatattaaatagcttggcagcaacaggactaggatgagtagcagcacgttccttatatgtagcttcgacatgatttatcttcgtttcct gcaggttttgttctgtgcagttgggttaagaatactgggcaatttcatgtttcttcaacactacatatgcgtatatataccaatctaagtctgtg ctccttccttcgttcttccttctgttcggagattaccgaatcaaaaaaatttcaaagaaaccgaaatcaaaaaaaagaataaaaaaaaa atgatgaattgaattgaaaagctgtggtatggtgcactctcagtacaatctgctctgatgccgcatagttaagccagccccgacacccg ccaacacccgctgacgcgccctgacgggcttgtctgctcccggcatccgcttacagacaagctgtgaccgtctccgggagctgcatgt gtcagaggtttcaccgtcatcaccgaaacgcgcga

\section{pRS416-BXG-altTAG-TyrOmeRS}

Note: original restriction enzyme sites Xbal and Sacll were eliminated at 3' and 5' ends intentionally due to presence of both restriction sites in TyrOmeRS-tRNA ${ }_{C U A}{ }^{\text {Tyr }}$ insert.

\section{GPD Promoter, TyrOmeRS, CYC1 terminator, tRNACUA ${ }^{\text {Tyr }}$, SNR52 promoter, GAL1, BFP, linker, TAG codon, GFP}

gacgaaagggcctcgtgatacgcctattttataggttaatgtcatgataataatggtttcttaggacggatcgcttgcctgtaacttacacg cgcctcgtatctttaatgatggaataatttgggaatttactctgtgtttatttattttatgtttgtatttggattttagaaagtaaataaagaaggt agaagagttacggaatgaagaaaaaaaaataaacaaaggtttaaaaaattcaacaaaaagcgtactttacatatatatttattagac aagaaaagcagattaaatagatatacattcgattaacgataagtaaaatgtaaaatcacaggatttcgtgtgtggtcttctacacagac aagatgaaacaattcggcattaatacctgagagcaggaagagcaagataaaaggtagtatttgttggcgatcccctagagtcttttac atcttcggaaaacaaaaactatttttctttaatttcttttttactttctatttttaatttatatatttatattaaaaaatttaaattataattattttatag cacgtgatgaaaaggacccaggtggcacttttcggggaaatgtgcgcggaacccctatttgtttatttttctaaatacattcaaatatgtat ccgctcatgagacaataaccctgataaatgcttcaataatattgaaaaaggaagagtatgagtattcaacatttccgtgtcgcccttattc cctttttgcggcatttgccttcctgttttgctcacccagaaacgctggtgaaagtaaaagatgctgaagatcagttgggtgcacgagtg ggttacatcgaactggatctcaacagcggtaagatccttgagagtttcgccccgaagaacgtttccaatgatgagcacttttaaagttct gctatgtggcgcggtattatcccgtattgacgccggg caagagcaactcggtcgccgcatacactattctcagaatgacttggttgagta ctcaccagtcacagaaaagcatcttacggatggcatgacagtaagagaattatgcagtgctgccataaccatgagtgataacactgc ggccaacttacttctgacaacgatcggaggaccgaaggagctaaccgctttttgcacaacatgggggatcatgtaactcgccttgatc gttgggaaccggagctgaatgaagccataccaaacgacgagcgtgacaccacgatgcctgtagcaatggcaacaacgttgcgca aactattaactggcgaactacttactctagcttcccggcaacaattaatagactggatggaggcggataaagttgcaggaccacttctg cgctcggcccttccggctggctggtttattgctgataaatctggagccggtgagcgtgggtctcgcggtatcattgcagcactggggcca gatggtaagccctcccgtatcgtagttatctacacgacggggagtcaggcaactatggatgaacgaaatagacagatcgctgagata ggtgcctcactgattaagcattggtaactgtcagaccaagtttactcatatatactttagattgatttaaaacttcattttaatttaaaaggatc taggtgaagatcctttttgataatctcatgaccaaaatcccttaacgtgagtttcgttccactgagcgtcagaccccgtagaaaagatca aaggatcttcttgagatccttttttctgcgcgtaatctgctgcttgcaaacaaaaaaaccaccgctaccagcggtggtttgtttgccggatc aagagctaccaactcttttccgaaggtaactggcttcagcagagcgcagataccaaatactgtccttctagtgtagccgtagttaggcc accacttcaagaactctgtagcaccgcctacatacctcgctctgctaatcctgttaccagtggctgctgccagtggcgataagtcgtgtct 
taccgggttggactcaagacgatagttaccggataaggcgcagcggtcgggctgaacggggggttcgtgcacacagcccagcttgg agcgaacgacctacaccgaactgagatacctacagcgtgagctatgagaaagcgccacgcttcccgaagggagaaaggcggac aggtatccggtaagcggcagggtcggaacaggagagcgcacgagggagcttccagggggaaacgcctggtatctttatagtcctgt cgggtttcgccacctctgacttgagcgtcgattttgtgatgctcgtcaggggggcggagcctatggaaaaacgccagcaacgcggcc ttttacggttcctggccttttgctggcctttgctcacatgttcttcctgcgttatccctgattctgtggataaccgtattaccgcctttgagtga gctgataccgctcgccgcagccgaacgaccgagcgcagcgagtcagtgagcgaggaagcggaagagcgcccaatacgcaaac cgcctctccccgcgcgttggccgattcattaatgcagctggcacgacaggtttcccgactggaaagcgggcagtgagcgcaacgca attaatgtgagttacctcactcattaggcaccccaggctttacactttatgcttccggctcctatgttgtgtggaattgtgagcggataacaat ttcacacaggaaacagctatgaccatgattacgccaagcgcgcaattaaccctcactaaagggaacaaaagctggagctccaccg cCCTAGGgagctcagtttatcattatcaatactcgccatttcaaagaatacgtaaataattaatagtagtgattttcctaactttatttagt caaaaaattagccttttaattctgctgtaacccgtacatgcccaaaatagggggcgggttacacagaatatataacatcgtaggtgtctg ggtgaacagtttattcctggcatccactaaatataatggagcccgcttttaagctggcatccagaaaaaaaaagaatcccagcacca aaatattgtttcttcaccaaccatcagttcataggtccattctcttagcgcaactacagagaacaggggcacaaacaggcaaaaaac gggcacaacctcaatggagtgatgcaacctgcctggagtaaatgatgacacaaggcaattgacccacgcatgtatctatctcattttctt acaccttctattaccttctgctctctctgatttggaaaaagctgaaaaaaaaggttgaaaccagttccctgaaattattcccctacttgacta ataagtatataaagacggtaggtattgattgtaattctgtaaatctatttcttaaacttcttaaattctacttttatagttagtctttttttagttttaa aacaccagaacttagtttcgacggattctagaactagtatggagattttgatggcaagcagtaacttgattaaacaattgcaagagcgg gggctggtagcccaggtgacggacgaggaagcgttagcagagcgactggcgcaaggcccgatcgcgctcacctgcggcttcgatc ctaccgctgacagcttgcatttggggcatcttgttccattgttatgcctgaaacgcttccagcaggcgggccacaagccggttgcgctggt aggcggcgcgacgggtctgattggcgacccgagcttcaaagctgccgagcgtaagctgaacactgaagaaactgttcaggagtgg gtggacaaaatccgcaagcaggttgcccottcctcgatttcgactgtggagaaaactctgctatcgcggcgaacaactatgactggtt cggcaatatgaatgtgctgaccttcctgcgcgatattggcaaacacttctccgttaaccagatgatcaacaaagaagcggttaagcag cgtctcaaccgtgaagatcaggggatttcgttcactgagtttcctacaacctgttgcagggttataccatggcctgtctgaacaaacagt acggtgtggtgctgcaaattggtggttctgaccagtggggtaacatcacttctggtatcgacctgacccgtcgtctgcatcagaatcaggt gtttggcctgaccgttccgctgatcactaaagcagatggcaccaaatttggtaaaactgaaggcggcgcagtctggttggatccgaag aaaaccagcccgtacaaattctaccagttctggatcaacactgcggatgccgacgtttaccgcttcctgaagttcttcacctttatgagca ttgaagagatcaacgcctggaagaagaagataaaaacagcggtaaagcaccgcgcgcccagtatgtactggcggagcaggtg actcgtctggttcacggtgaagaaggtttacaggcggcaaaacgtattaccgaatgcctgttcagcggttcttgagtgcgctgagtgaa gcggacttcgaacagctggcgcaggacggcgtaccgatggttgagatggaaaagggcgcagacctgatgcaggcactggtcgatt ctgaactgcaaccttcccgtggtcaggcacgtaaaactatcgcctccaatgccatcaccattaacggtgaaaaacagtccgatcctga atacttctttaaagaagaagatcgtctgtttggtcgttttaccttactgcgtcgtggcaaaaagaattactgtctgatttgctggaaataact agtcatgtaattagttatgtcacgcttacattcacgccctccccccacatccgctctaaccgaaaaggaaggagttagacaacctga: gccggtacccctggaaggtgccactcccac tgtccttcctaataaaatgaggaaattgcatcgcattgtctgagtaggtgtcattctattctggggggtggggtggggcaggacagcaa gggggaggattgggaagacaatagcaggcatgctggggatgcggtgggctctatggcttctgaggcggaaagaaccagctgggg ctctagggggtatccccacgcgccctgtagcggcgcattaagcgcggcgggtgtggtggttacgcgcagcgtgaccgctacacttgc cagcgccctagcgcccg cactagagtcgacagacataaaaaacaaaaaaatggtgggggaaggattcgaaccttcgaagtcgat gacggcagatttagagtctgctccctttggccgctcgggaaccccaccgatcatttatctttcactgcggagaagtttcgaacgccgaaa catgcgcaccaactttcacttctacagcgtttgaccaaaatctttgaacagaacattgtagggtgtgaaaaaatgcgcacctttaccgct agcccaagagggcactacaaaatctagagttgtacttcaaacgtacatgtaatcaccttgtatatactcgaaagaaaacatcaagttc tgtataaatatgagtgaaagcataatcatacattatcttttcaaagactgcaggaattcgatatcaagcttCCCGGGctagagctggt accaattccttgaattttcaaaaattcttacttttttttggatggacgcaaagaagtttaataatcatattacatggcattaccaccatatacat atccatatctaatcttacttatatgttgtggaaatgtaaagagccccattatcttagcctaaaaaaaccttctctttggaactttcagtaatac 
gcttaactgctcattgctatattgaagta

atttctggggtaattaatcagcgaagcgatgattttgatctattaacagatatataaatgcaaaaactgcataac cactttaactaatactttcaacattttcggtttgtattacttcttattcaaatgtaataaaagtatcaacaaaaaattgttaatatacctctatactt taacgtcaaggagaaaaaaccccggatcgaattccctacttcatacattttcaattaag

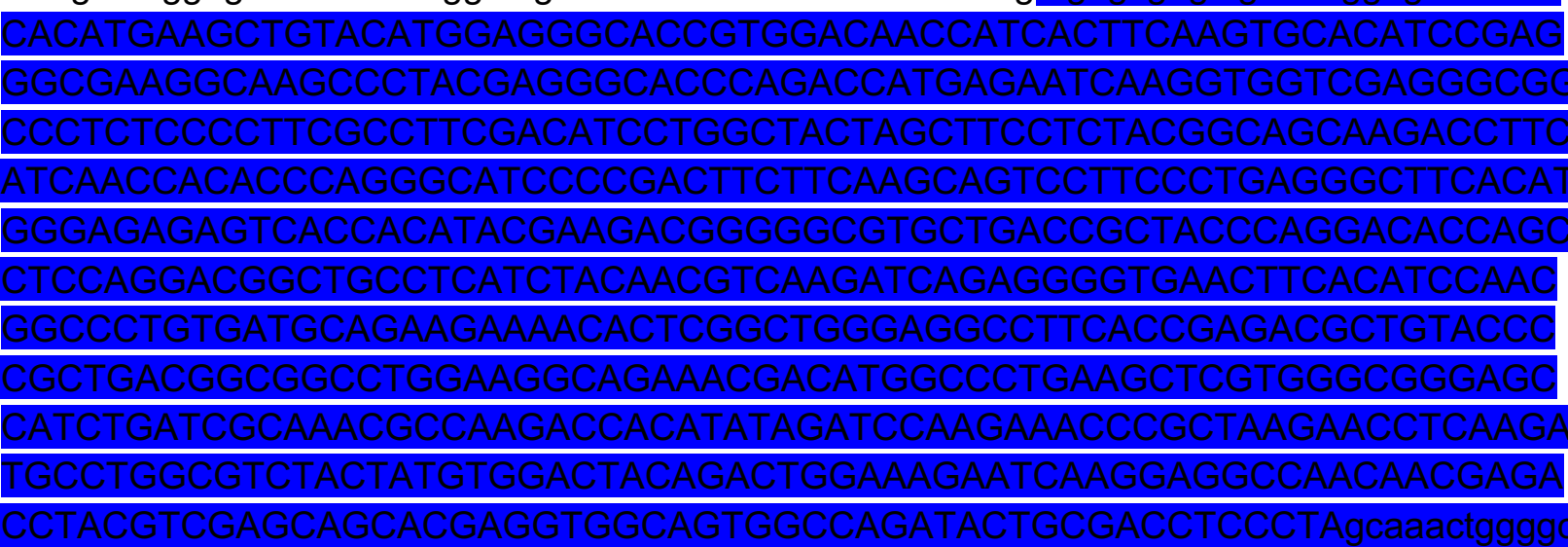

atgggaTAGgctggctccgctgctggttctggcgaatacgcatccaagggcgaggagctctttactggcgtagtac

caattctcgtagagctcgatggcgatgtaaatggccataagtttccgtacgcggcgagggcgagggcgatgcaactaacggcaagc tcactctcaagtttatttgtactactggcaagctcccagtaccatggccaactctcgtaactactctgacctatggcgtacaatgttttcccg ctatccagatcacatgaagcaacatgattttttaagtccgcaatgccagagggctatgtacaagagcgcactattagctttaaggatga tggcacctataagactcgcgcagaggtaaagtttgagggcgatactctcgtaaatcg cattgagctcaagggcattgattttaaggagg atggcaatattctcggccataagctggagtataatttcaattcccataatgtatatattaccgcagataagcaaaagaatggcattaagg cgaattttaagattcgccataatgtggaggatggctccgtacaactcg cagatcattatcaacaaaatactccaattggcgatggccca gtactcctcccagataatcattatctctccactcaatccgtgctctccaaagatccaaatgagaagcgcgatcacatggtactcctggag tttgtaactgcagcaggcattactcatgg catggatgagctctataagctcgagcaccaccaccaccaccactaataatagctcgagat ctgataacaacagtgtagatgtaacaaaatcgactttgttcccactgtacttttagctcgtacaaaatacaatatactttcatttctccgtaa acaacatgtttcccatgtaatatccttttctattttcgttccgttaccaactttacacatactttatatagctattcacttctatacactaaaaaa ctaagacaattttaattttgctgcctgccatatttcaatttgttataaattcctataatttatcctattagtagctaaaaaaagatgaatgtgaat cgaatcctaagagaattgagctccaattcggtcgacctcgagggggggcccggtacccaattcgccctatagtgagtcgtattacgcg cgctcactggccgtcgttttacaacgtcgtgactgggaaaaccctggcgttacccaacttaatcgccttgcagcacatcccctttcgcc agctggcgtaatagcgaagaggcccgcaccgatcgcccttcccaacagttgcgcagcctgaatggcgaatggcgcgacgcgccct gtagcggcgcattaagcgcggcgggtgtggtggttacgcgcagcgtgaccgctacacttgccagcgccctagcgcccgctcctttcgc tttctcccttccttctcgccacgttcgccggcttccccgtcaagctctaaatcggggg ctcctttagggttccgatttagtgctttacggca cctcgaccccaaaaaacttgattagggtgatggttcacgtagtgggccatcgccctgatagacggttttcgccctttgacgttggagtcc acgttcttaatagtggactcttgttccaaactggaacaacactcaaccctatctcggtctattctttgatttataagggattttgccgatttcg gcctattggttaaaaaatgagctgatttaacaaaaatttaacgcgaattttaacaaaatattaacgtttacaatttcctgatgcggtattttct ccttacgcatctgtgcggtatttcacaccgcatagggtaataactgatataattaaattgaagctctaatttgtgagtttagtatacatgcattt acttataatacagtttttagttttgctggccgcatcttctcaaatatgcttcccagcctgctttctgtaacgttcaccctctaccttagcatccct tccctttgcaaatagtcctcttccaacaataataatgtcagatcctgtagagaccacatcatccacggttctatactgttgacccaatgcgt ctcccttgtcatctaaacccacaccgggtgtcataatcaaccaatcgtaaccttcatctcttccacccatgtctctttgagcaataaagccg ataacaaaatctttgtcgctcttcgcaatgtcaacagtacccttagtatattctccagtagatagggagccttgcatgacaattctgctaa catcaaaaggcctctaggttcctttgttacttcttctgccgcctgcttcaaaccgctaacaatacctgggcccaccacaccgtgtgcattcg taatgtctgcccattctgctattctgtatacacccgcagagtactg caatttgactgtattaccaatgtcagcaaatttctgtcttcgaagagt 
aaaaaattgtacttggcggataatgcctttagcggcttaactgtgccctccatggaaaaatcagtcaagatatccacatgtgttttagtaa acaaatttgggacctaatgcttcaactaactccagtaattccttggtggtacgaacatccaatgaagcacacaagtttgtttgcttttcgtg catgatattaaatagcttggcagcaacaggactaggatgagtagcagcacgttccttatatgtagcttcgacatgatttatcttcgtttcct gcaggttttgttctgtgcagttgggttaagaatactgggcaatttcatgtttcttcaacactacatatgcgtatatataccaatctaagtctgtg ctccttccttcgttcttccttctgttcggagattaccgaatcaaaaaaattcaaagaaaccgaaatcaaaaaaaagaataaaaaaaaa atgatgaattgaattgaaaagctgtggtatggtgcactctcagtacaatctgctctgatgccgcatagttaagccagccccgacacccg ccaacacccgctgacgcgccctgacgggcttgtctgctcccggcatccgcttacagacaagctgtgaccgtctccgggagctgcatgt gtcagaggtttcaccgtcatcaccgaaacgcgcga

\section{pCTCON2-FAPB2.3.6-LeuOmeRS}

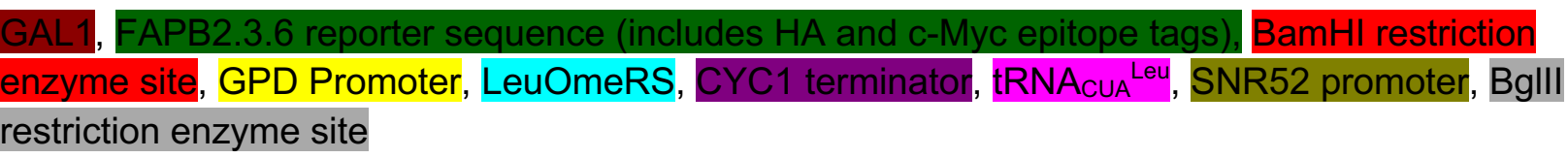

gacgaaagggcctcgtgatacgcctattttataggttaatgtcatgataataatggtttcttagacggatcgcttgcctgtaacttacacgc gcctcgtatcttttaatgatggaataatttgggaatttactctgtgtttatttattttatgtttgtatttggattttagaaagtaaataaagaaggta gaagagttacggaatgaagaaaaaaaaataaacaaaggtttaaaaaatttcaacaaaaagcgtactttacatatatatttattagaca agaaaagcagattaaatagatatacattcgattaacgataagtaaaatgtaaaatcacaggatttcgtgtgtggtcttctacacagaca agatgaaacaattcggcattaatacctgagagcaggaagagcaagataaaaggtagtatttgttggcgatccccctagagtcttttaca tcttcggaaaacaaaaactatttttctttaatttcttttttactttctattttaatttatatatttatattaaaaaatttaaattataattattttatagc acgtgatgaaaaggacccaggtgg cacttttcggggaaatgtgcgcggaacccctatttgtttattttctaaatacattcaaatatgtatc cgctcatgagacaataaccctgataaatgcttcaataatattgaaaaaggaagagtatgagtattcaacatttccgtgtcgcccttattcc ctttttgcggcatttgccttcctgtttttgctcacccagaaacgctggtgaaagtaaaagatgctgaagatcagttgggtgcacgagtgg gttacatcgaactggatctcaacagcggtaagatccttgagagtttcgccccgaagaacgttttccaatgatgagcacttttaaagttctg ctatgtggcgcggtattatcccgtattgacgccgggcaagagcaactcggtcgccgcatacactattctcagaatgacttggttgagtac tcaccagtcacagaaaagcatcttacggatggcatgacagtaagagaattatgcagtgctgccataaccatgagtgataacactgcg gccaacttacttctgacaacgatcggaggaccgaaggagctaaccgctttttgcacaacatgggggatcatgtaactcgccttgatcg ttgggaaccggagctgaatgaagccataccaaacgacgagcgtgacaccacgatgcctgtagcaatggcaacaacgttgcgcaa actattaactggcgaactacttactctagcttcccggcaacaattaatagactggatggaggcggataaagttgcaggaccacttctgc gctcggcccttccggctggctggtttattgctgataaatctggagccggtgagcgtgggtctcgcggtatcattgcagcactggggccag atggtaagccctcccgtatcgtagttatctacacgacggggagtcaggcaactatggatgaacgaaatagacagatcgctgagatag gtgcctcactgattaag cattggtaactgtcagaccaagtttactcatatatactttagattgatttaaaacttcattttaatttaaaaggatct aggtgaagatccttttgataatctcatgaccaaaatcccttaacgtgagttttcgttccactgagcgtcagaccccgtagaaaagatcaa aggatcttcttgagatccttttttctgcgcgtaatctgctgcttgcaaacaaaaaaaccaccgctaccagcggtggtttgtttgccggatca agagctaccaactcttttccgaaggtaactggcttcagcagagcgcagataccaaatactgttcttctagtgtagccgtagttaggcca ccacttcaagaactctgtagcaccgcctacatacctcgctctgctaatcctgttaccagtggctgctgccagtggcgataagtcgtgtctt accgggttggactcaagacgatagttaccggataaggcgcagcggtcgggctgaacggggggttcgtgcacacagcccagcttgg agcgaacgacctacaccgaactgagatacctacagcgtgagctatgagaaagcgccacgcttcccgaagggagaaaggcggac aggtatccggtaagcggcagggtcggaacaggagagcgcacgagggagcttccagggggaaacgcctggtatcttatagtcctgt cgggtttcgccacctctgacttgagcgtcgattttgtgatgctcgtcaggggggcggagcctatggaaaaacgccagcaacgcggcc ttttacggttcctggccttttgctggccttttgctcacatgttctttcctgcgttatccctgattctgtggataaccgtattaccgcctttgagtga gctgataccgctcgccgcagccgaacgaccgagcgcagcgagtcagtgagcgaggaagcggaagagcgcccaatacgcaaac cgcctctccccgcgcgttggccgattcattaatgcagctggcacgacaggtttcccgactggaaagcgggcagtgagcgcaacgca 
attaatgtgagttagctcactcattaggcaccccaggctttacactttatgcttccggctcgtatgttgtgtggaattgtgagcggataacaa tttcacacaggaaacagctatgaccatgattacgccaagctcgaaattaaccctcactaaagggaacaaaagctggtaccaattcctt gaatttcaaaaattcttacttttttttggatggacgcaaagaagtttaataatcatattacatggcattaccaccatatacatatccatatcta atcttacttatatgttgtggaaatgtaaagagccccattatcttagcctaaaaaaaccttctctttggaactttcagtaatacgcttaactgctc attgctatattgaagta

atttctggggtaattaatcagcgaagcg

ctcgaggagctcagtttatcattatcaatactcgccatttcaaagaatacgtaa

ataattaatagtagtgattttcctaactttatttagtcaaaaaattagccttttaattctgctgtaacccgtacatgcccaaaatagggggcgg gttacacagaatatataacatcgtaggtgtctgggtgaacagtttattcctggcatccactaaatataatggagcccgcttttaagctggc atccagaaaaaaaaagaatcccagcaccaaaatattgtttcttcaccaaccatcagttcataggtccattctcttagcgcaactacag agaacaggggcacaaacaggcaaaaaacgggcacaacctcaatggagtgatgcaacctgcctggagtaaatgatgacacaag gcaattgacccacgcatgtatctatctcatttcttacaccttctattaccttctgctctctctgatttggaaaaagctgaaaaaaaaggttga aaccagttccctgaaattattcccctacttgactaataagtatataaagacggtaggtattgattgtaattctgtaaatctatttcttaaacttct taaattctacttttatagttagtctttttttagttttaaaacaccagaacttagtttcgacggattctagaactagtatggagatttCCATGG AAGAGCAATACCGCCCGGAAGAGATAGAATCCAAAGTACAGCTTCATTGGGATGAGAAGC GCACATTTGAAGTAACCGAAGACGAGAGCAAAGAGAAGTATTACTGCCTGTCTTTGGAACC CTATCCTTCTGGTCGACTACACATGGGCCACGTACGTAACTACACCATCGGTGACGTGATC GCCCGCTACCAGCGTATGCTGGGCAAAAACGTCCTGCAGCCGATCGGCTGGGACGCGTT TGGTCTGCCTGCGGAAGGCGCGGCGGTGAAAAACAACACCGCTCCGGCACCGTGGACGT ACGACAACATCGCGTATATGAAAAACCAGCTCAAAATGCTGGGCTTTGGTTATGACTGGAG CCGCGAGCTGGCAACCTGTACGCCGGAATACTACCGTTGGGAACAGAAATTCTTCACCGA GCTGTATAAAAAAGGCCTGGTATATAAGAAGACTTCTGCGGTCAACTGGTGCCCGAACGAC CAGACCGTACTGGCGAACGAACAAGTTATCGACGGCTGCTGCTGGCGCTGCGATACCAAA GTTGAACGTAAAGAGATCCCGCAGTGGTTTATCAAAATCACTGCTTACGCTGACGAGCTGC TCAACGATCTGGATAAACTGGATCACTGGCCAGACACCGTTAAAACCATGCAGCGTAACTG GATCGGTCGTTCCGAAGGCGTGGAGATCACCTTCAACGTTAACGACTATGACAACACGCT GACCGTTTACACTACCCGCCCGGACGCTTTTATGGGTTGTACCTACCTGGCGGTAGCTGC GGGTCATCCGCTGGCGCAGAAAGCGGCGGAAAATAATCCTGAACTGGCGGCCTTTATTGA 
CGAATGCCGTAACACCAAAGTTGCCGAAGCTGAAATGGCGACGATGGAGAAAAAAGGCGT CGATACTGGCTTTAAAGCGGTTCACCCATTAACGGGCGAAGAAATTCCCGTTTGGGCAGCA AACTTCGTATTGATGGAGTACGGCACGGGCGCAGTTATGGCGGTACCGGGGCACGACCA GCGCGACTACGAGTTTGCCTCTAAATACGGCCTGAACATCAAACCGGTTATCCTGGCAGCT GACGGCTCTGAGCCAGATCTTTCTCAGCAAGCCCTGACTGAAAAAGGCGTGCTGTTCAACT CTGGCGAGTTCAACGGTCTTGACCATGAAGCGGCCTTCAACGCCATCGCCGATAAACTGA CTGCGATGGGCGTTGGCGAGCGTAAATTGAACTACCGCCTGCGCGACTGGGGTGTTTCCC GTCAGCGTTACTGGGGCGCGCCGATTCCGATGGTGACGCTGGAAGACGGTACCGTAATG CCGACCCCGGACGACCAGCTGCCGGTGATCCTGCCGGAGGATGTGGTAATGGACGGCAT TACCAGCCCGATTAAAGCAGATCCGGAGTGGGCGAAAACTACCGTTAACGGTATGCCAGC ACTGCGTGAAACCGACACTTTCGACACCTTTATGGAGTCCTCCTGGAGATATGCGCGCTAC ACTTGCCCGCAGTACAAAGAAGGTATGCTGGATTCCGAAGCGGCTAACTACTGGCTGCCG GTGGATATCGCTATTGGTGGTATTGAACACGCCATTATGGGTCTGCTCTACTTCCGCTTCTT CCACAAACTGATGCGTGATGCAGGCATGGTGAACTCTGACGAACCAGCGAAACAGTTGCT GTGTCAGGGTATGGTGCTGGCAGATGCCTTCTACTATGTTGGCGAAAACGGCGAACGTAA CTGGGTTTCCCCGGTTGATGCTATCGTTGAACGTGACGAGAAAGGCCGTATCGTGAAAGC GAAAGATGCGGCAGGCCATGAACTGGTTTATACCGGCATGAGCAAAATGTCCAAGTCGAA GAACAACGGTATCGACCCGCAGGTGATGGTTGAACGTTACGGCGCGGACACCGTTCGTCT GTTTATGATGTTTGCTTCTCCGGCTGATATGACTCTCGAATGGCAGGAATCCGGTGTGGAA GGGGCTAACCGCTTCCTGAAACGTGTCTGGAAACTGGTTTACGAGCACACAGCAAAAGGT GATGTTGCGGCACTGAACGTTGATGCGCTGACTGAAAATCAGAAAGCGCTGCGTCGCGAT GTGCATAAAACGATCGCTAAAGTGACCGATGATATCGGCCGTCGTCAGACCTTCAACACCG CAATTGCGGCGATTATGGAGCTGATGAACAAACTGGCGAAAGCACCAACCGATGGCGAGC AGGATCGCGCTCTGATGCAGGAAGCACTGCTGGCCGTTGTCCGTATGCTTAACCCGTTCA CCCCGCACATCTGCTTCACGCTGTGGCAGGAACTGAAAGGCGAAGGCGATATCGACAACG CGCCGTGGCCGGTTGCTGACGAAAAAGCGATGGTGGAAGACTCCACGCTGGTCGTGGTG CAGGTTAACGGTAAGGTCCGTGCCAAAATCACCGTTCCGGTGGACGCAACGGAAGAACAG GTTCGCGAACGTGCTGGCCAGGAACATCTGGTAGCAAAATATCTTGATGGCGTTACTGTAC GTAAAGTGATTTACGTACCAGGTAAACTCCTCAATCTGGTCGTTGGCTAA

ttcacgccctccccccacatccgctctaaccgaaaaggaaggagttagacaacctgaagtctaggtccctatttattttttatagttatgt ccggtaccctggaaggtgccactcccactgtcctttcctaataaaatgaggaaattgca tcgcattgtctgagtaggtgtcattctattctggggggtggggtggggcaggacagcaagggggaggattgggaagacaatagcagg catgctggggatgcggtgggctctatggcttctgaggcggaaagaaccagctggggctctagggggtatccccacgcgcctgtagc ggcgcattaagcgcggcgggtgtggtggttacgcgcagcgtgaccgctacacttgccagcgcctagcgcccgcactagagACG TCCCGCGGGGCGGCTCTAGACATAAAAAACAAAAAAATACCCGGAGCGGGACTTGAACCC GCACAGCGCGAACGCCGAGGGATTTAGAATCCCTTGTGTCTACCGATTCCACCATCCGGG CTATTTAATTGTTGAAGAAAGAGTATACTACATAACACATATACAATTGAAAAAGAGGCTAG CAGCTTACCGGTCGGGATGGCTGCAGgat ccaactttcacttctacagcgtttgaccaaaatctttgaacagaacattgtagggtgtgaaaaaatgcgcacctttaccgctagcccaa gagggcactacaaaatctagagttgtacttcaaacgtacatgtaatcaccttgtatatactcgaaagaaaacatcaagtttctgtataaat atgagtgaaagcataatcatacattatctttcaaagactgcagAGATCTgataacaacagtgtagatgtaacaaaatcgactttgtt cccactgtacttttagctcgtacaaaatacaatatacttttcatttctccgtaaacaacatgtttcccatgtaatatccttttctatttttcgttccgt taccaactttacacatactttatatagctattcacttctatacactaaaaaactaagacaattttaatttgctgcctgccatatttcaatttgttat 
aaattcctataatttatcctattagtagctaaaaaaagatgaatgtgaatcgaatcctaagagaattgagctccaattcgccctatagtga gtcgtattacaattcactggccgtcgttttacaacgtcgtgactgggaaaaccctggcgttacccaacttaatcgccttgcagcacatccc cctttcgccagctggcgtaatagcgaagaggcccgcaccgatcgcctttcccaacagttgcgcagcctgaatggcgaatggacgcg ccctgtagcggcg cattaagcgcggcgggtgtggtggttacgcgcagcgtgaccgctacacttgccagcgccctagcgcccgctcctt tcgctttcttcccttcctttctcgccacgttcgccggctttccccgtcaagctctaaatcggggg ctccetttagggttccgatttagtgctttac ggcacctcgacccaaaaaacttgattagggtgatggttcacgtagtgggccatcgccctgatagacggttttcgccctttgacgttgg agtccacgttctttaatagtggactcttgttccaaactggaacaacactcaaccctatctcggtctattctttgatttataagggattttgccg atttcggcctattggttaaaaaatgagctgatttaacaaaaatttaacgcgaattttaacaaaatattaacgcttacaatttcctgatgcggt atttctccttacgcatctgtgcggtatttcacaccgcatagatcggcaagtgcacaaacaatacttaaataaatactactcagtaataac ctatttcttagcatttttgacgaaatttgctattttgttagagtcttttacaccatttgtctccacacctccgcttacatcaacaccaataacgcca tttaatctaagcgcatcaccaacattttctggcgtcagtccaccagctaacataaaatgtaagctttcggggctctcttgccttccaaccca gtcagaaatcgagttccaatccaaaagttcacctgtcccacctgcttctgaatcaaacaagggaataaacgaatgaggtttctgtgaag ctgcactgagtagtatgttgcagtcttttggaaatacgagtcttttaataactggcaaaccgaggaactcttggtattcttgccacgactcat ctccatgcagttggacgatatcaatgccgtaatcattgaccagagccaaaacatcctccttaggttgattacgaaacacgccaaccaa gtatttcggagtgcctgaactattttatatgcttttacaagacttgaaattttccttgcaataaccgggtcaattgttctctttctattgggcaca catataatacccagcaagtcagcatcggaatctagag cacattctgcggcctctgtgctctgcaagccgcaaactttcaccaatggac cagaactacctgtgaaattaataacagacatactccaagctgccttgtgtgcttaatcacgtatactcacgtgctcaatagtcaccaatg ccctccctcttggccctctccttttctttttcgaccgaattaattcttaatcggcaaaaaaagaaaagctccggatcaagattgtacgtaag gtgacaagctatttttcaataaagaatatcttccactactgccatctggcgtcataactgcaaagtacacatatattacgatgctgttctatta aatgcttcctatattatatatatagtaatgtcgtgatctatggtgcactctcagtacaatctgctctgatgccgcatagttaagccagccccg acacccgccaacacccgctgacgcgccctgacgggettgtctgctcccggcatccgcttacagacaagctgtgaccgtctccgggag ctgcatgtgtcagaggttttcaccgtcatcaccgaaacgcgcga

\section{pCTCON2-FAPB2.3.6L1TAG-LeuOMeRS}

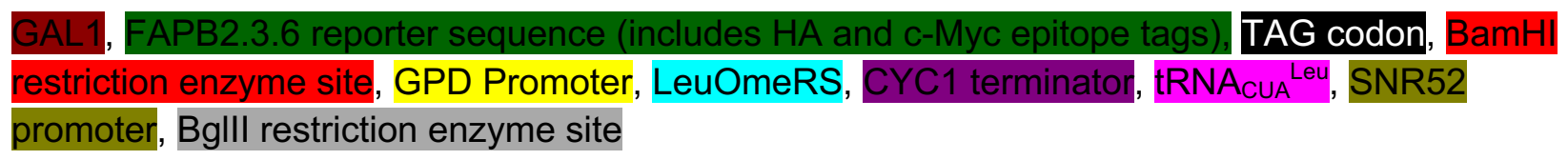

gacgaaagggcctcgtgatacgcctattttataggttaatgtcatgataataatggtttcttagacggatcgcttgcctgtaacttacacgc gcctcgtatcttttaatgatggaataatttgggaatttactctgtgtttatttattttatgtttgtatttggattttagaaagtaaataaagaaggta gaagagttacggaatgaagaaaaaaaaataaacaaaggtttaaaaaatttcaacaaaaagcgtactttacatatatatttattagaca agaaaagcagattaaatagatatacattcgattaacgataagtaaaatgtaaaatcacaggatttcgtgtgtggtcttctacacagaca agatgaaacaattcggcattaatacctgagagcaggaagagcaagataaaaggtagtatttgttggcgatccccctagagtctttaca tcttcggaaaacaaaaactattttttctttaatttcttttttactttctatttttaatttatatatttatattaaaaaatttaaattataattattttatagc acgtgatgaaaaggacccaggtgg cacttttcggggaaatgtgcgcggaacccctatttgtttattttctaaatacattcaaatatgtatc cgctcatgagacaataaccctgataaatgcttcaataatattgaaaaaggaagagtatgagtattcaacatttccgtgtcgcccttattcc ctttttgcggcattttgccttcctgttttgctcacccagaaacgctggtgaaagtaaaagatgctgaagatcagttgggtgcacgagtgg gttacatcgaactggatctcaacagcggtaagatccttgagagtttcgccccgaagaacgttttccaatgatgag cacttttaaagttctg ctatgtggcgcggtattatcccgtattgacgccggg caagagcaactcggtcgccgcatacactattctcagaatgacttggttgagtac tcaccagtcacagaaaagcatcttacggatggcatgacagtaagagaattatgcagtgctgccataaccatgagtgataacactgcg gccaacttacttctgacaacgatcggaggaccgaaggagctaaccgctttttgcacaacatgggggatcatgtaactcgccttgatcg ttgggaaccggagctgaatgaagccataccaaacgacgagcgtgacaccacgatgcctgtagcaatggcaacaacgttgcgcaa actattaactggcgaactacttactctagcttcccggcaacaattaatagactggatggaggcggataaagttgcaggaccacttctgc 
gctcggcccttccggctggctggtttattgctgataaatctggagccggtgagcgtgggtctcgcggtatcattgcagcactggggccag atggtaagccctcccgtatcgtagttatctacacgacggggagtcaggcaactatggatgaacgaaatagacagatcgctgagatag gtgcctcactgattaagcattggtaactgtcagaccaagtttactcatatatactttagattgatttaaaacttcattttaatttaaaaggatct aggtgaagatccttttgataatctcatgaccaaaatcccttaacgtgagttttcgttccactgagcgtcagaccccgtagaaaagatcaa aggatcttcttgagatccttttttctgcgcgtaatctgctgcttgcaaacaaaaaaaccaccgctaccagcggtggtttgtttgccggatca agagctaccaactcttttccgaaggtaactggettcagcagagcgcagataccaaatactgttcttctagtgtagccgtagttaggcca ccacttcaagaactctgtagcaccgcctacatacctcgctctgctaatcctgttaccagtggctgctgccagtggcgataagtcgtgtctt accgggttggactcaagacgatagttaccggataaggcgcagcggtcgggctgaacggggggttcgtgcacacagcccagcttgg agcgaacgacctacaccgaactgagatacctacagcgtgagctatgagaaagcgccacgcttcccgaagggagaaaggcggac aggtatccggtaagcggcagggtcggaacaggagagcgcacgagggagcttccagggggaaacgcctggtatcttatagtcctgt cgggtttcgccacctctgacttgagcgtcgattttgtgatgctcgtcaggggggcggagcctatggaaaaacgccagcaacgcggcc ttttacggttcctggccttttgctggccttttgctcacatgttcttcctgcgttatccctgattctgtggataaccgtattaccgccttgagtga gctgataccgctcgccgcagccgaacgaccgagcgcagcgagtcagtgagcgaggaagcggaagagcgcccaatacgcaaac cgcctctccccgcgcgttggccgattcattaatgcagctggcacgacaggtttcccgactggaaagcgggcagtgagcgcaacgca attaatgtgagttagctcactcattaggcacccaggctttacactttatgcttccggctcgtatgttgtgtggaattgtgagcggataacaa tttcacacaggaaacagctatgaccatgattacgccaagctcgaaattaaccctcactaaagggaacaaaagctggtaccaattcctt gaattttcaaaaattcttacttttttttggatggacgcaaagaagtttaataatcatattacatggcattaccaccatatacatatccatatcta atcttacttatatgttgtggaaatgtaaagagccccattatcttagcctaaaaaaaccttctcttggaactttcagtaatacgcttaactgctc attgctatattgaagta

atttctggggtaattaatcagcgaagcg

\section{TAG}

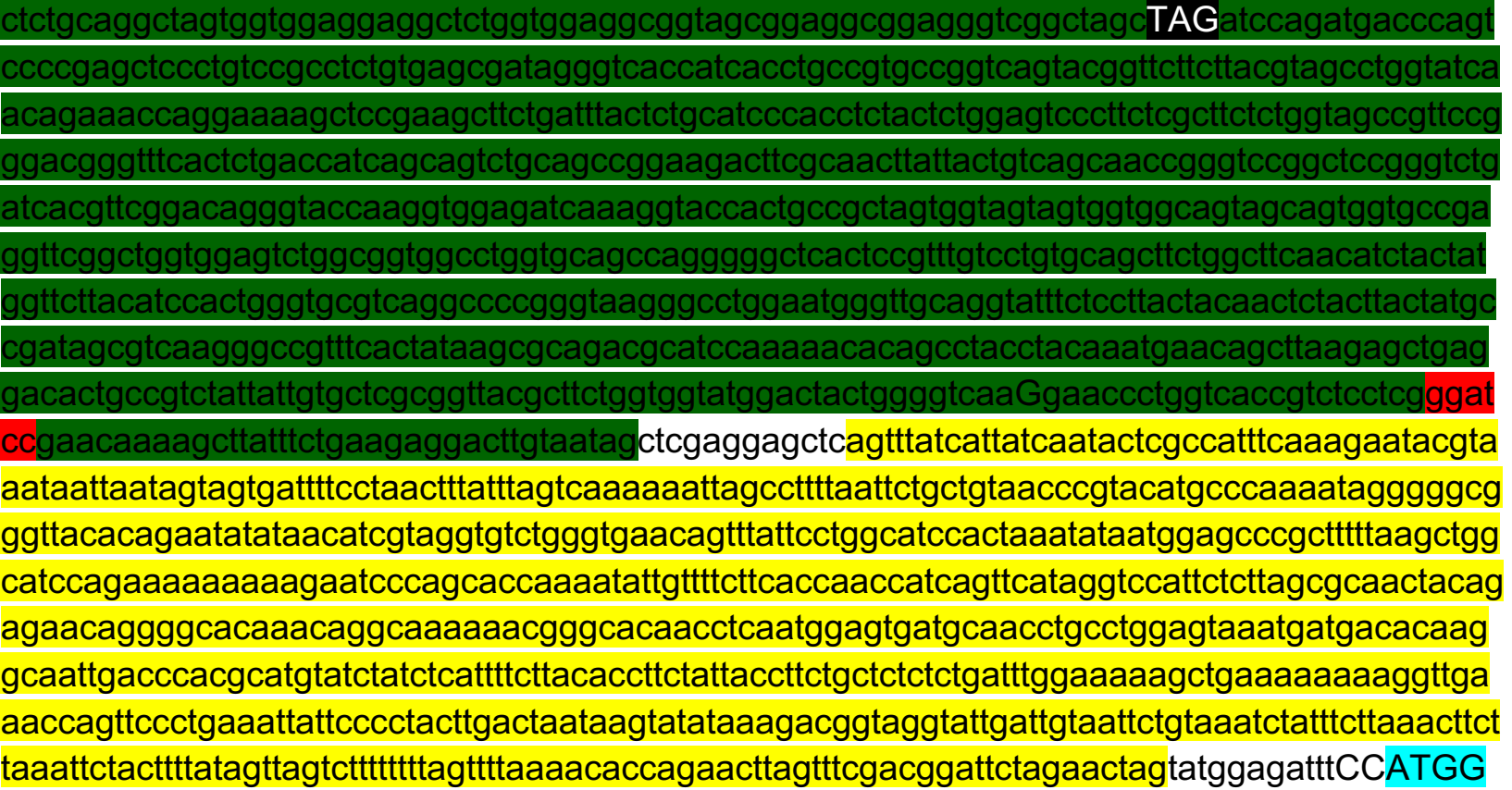


AAGAGCAATACCGCCCGGAAGAGATAGAATCCAAAGTACAGCTTCATTGGGATGAGAAGC GCACATTTGAAGTAACCGAAGACGAGAGCAAAGAGAAGTATTACTGCCTGTCTTTGGAACC CTATCCTTCTGGTCGACTACACATGGGCCACGTACGTAACTACACCATCGGTGACGTGATC GCCCGCTACCAGCGTATGCTGGGCAAAAACGTCCTGCAGCCGATCGGCTGGGACGCGTT TGGTCTGCCTGCGGAAGGCGCGGCGGTGAAAAACAACACCGCTCCGGCACCGTGGACGT ACGACAACATCGCGTATATGAAAAACCAGCTCAAAATGCTGGGCTTTGGTTATGACTGGAG CCGCGAGCTGGCAACCTGTACGCCGGAATACTACCGTTGGGAACAGAAATTCTTCACCGA GCTGTATAAAAAAGGCCTGGTATATAAGAAGACTTCTGCGGTCAACTGGTGCCCGAACGAC CAGACCGTACTGGCGAACGAACAAGTTATCGACGGCTGCTGCTGGCGCTGCGATACCAAA GTTGAACGTAAAGAGATCCCGCAGTGGTTTATCAAAATCACTGCTTACGCTGACGAGCTGC TCAACGATCTGGATAAACTGGATCACTGGCCAGACACCGTTAAAACCATGCAGCGTAACTG GATCGGTCGTTCCGAAGGCGTGGAGATCACCTTCAACGTTAACGACTATGACAACACGCT GACCGTTTACACTACCCGCCCGGACGCTTTTATGGGTTGTACCTACCTGGCGGTAGCTGC GGGTCATCCGCTGGCGCAGAAAGCGGCGGAAAATAATCCTGAACTGGCGGCCTTTATTGA CGAATGCCGTAACACCAAAGTTGCCGAAGCTGAAATGGCGACGATGGAGAAAAAAGGCGT CGATACTGGCTTTAAAGCGGTTCACCCATTAACGGGCGAAGAAATTCCCGTTTGGGCAGCA AACTTCGTATTGATGGAGTACGGCACGGGCGCAGTTATGGCGGTACCGGGGCACGACCA GCGCGACTACGAGTTTGCCTCTAAATACGGCCTGAACATCAAACCGGTTATCCTGGCAGCT GACGGCTCTGAGCCAGATCTTTCTCAGCAAGCCCTGACTGAAAAAGGCGTGCTGTTCAACT CTGGCGAGTTCAACGGTCTTGACCATGAAGCGGCCTTCAACGCCATCGCCGATAAACTGA CTGCGATGGGCGTTGGCGAGCGTAAATTGAACTACCGCCTGCGCGACTGGGGTGTTTCCC GTCAGCGTTACTGGGGCGCGCCGATTCCGATGGTGACGCTGGAAGACGGTACCGTAATG CCGACCCCGGACGACCAGCTGCCGGTGATCCTGCCGGAGGATGTGGTAATGGACGGCAT TACCAGCCCGATTAAAGCAGATCCGGAGTGGGCGAAAACTACCGTTAACGGTATGCCAGC ACTGCGTGAAACCGACACTTTCGACACCTTTATGGAGTCCTCCTGGAGATATGCGCGCTAC ACTTGCCCGCAGTACAAAGAAGGTATGCTGGATTCCGAAGCGGCTAACTACTGGCTGCCG GTGGATATCGCTATTGGTGGTATTGAACACGCCATTATGGGTCTGCTCTACTTCCGCTTCTT CCACAAACTGATGCGTGATGCAGGCATGGTGAACTCTGACGAACCAGCGAAACAGTTGCT GTGTCAGGGTATGGTGCTGGCAGATGCCTTCTACTATGTTGGCGAAAACGGCGAACGTAA CTGGGTTTCCCCGGTTGATGCTATCGTTGAACGTGACGAGAAAGGCCGTATCGTGAAAGC GAAAGATGCGGCAGGCCATGAACTGGTTTATACCGGCATGAGCAAAATGTCCAAGTCGAA GAACAACGGTATCGACCCGCAGGTGATGGTTGAACGTTACGGCGCGGACACCGTTCGTCT GTTTATGATGTTTGCTTCTCCGGCTGATATGACTCTCGAATGGCAGGAATCCGGTGTGGAA GGGGCTAACCGCTTCCTGAAACGTGTCTGGAAACTGGTTTACGAGCACACAGCAAAAGGT GATGTTGCGGCACTGAACGTTGATGCGCTGACTGAAAATCAGAAAGCGCTGCGTCGCGAT GTGCATAAAACGATCGCTAAAGTGACCGATGATATCGGCCGTCGTCAGACCTTCAACACCG CAATTGCGGCGATTATGGAGCTGATGAACAAACTGGCGAAAGCACCAACCGATGGCGAGC AGGATCGCGCTCTGATGCAGGAAGCACTGCTGGCCGTTGTCCGTATGCTTAACCCGTTCA CCCCGCACATCTGCTTCACGCTGTGGCAGGAACTGAAAGGCGAAGGCGATATCGACAACG CGCCGTGGCCGGTTGCTGACGAAAAAGCGATGGTGGAAGACTCCACGCTGGTCGTGGTG CAGGTTAACGGTAAGGTCCGTGCCAAAATCACCGTTCCGGTGGACGCAACGGAAGAACAG GTTCGCGAACGTGCTGGCCAGGAACATCTGGTAGCAAAATATCTTGATGGCGTTACTGTAC GTAAAGTGATTTACGTACCAGGTAAACTCCTCAATCTGGTCGTTGGCTAA 
ccggtaccctggaaggtgccactcccactgtcctttcctaataaaatgaggaaattgca tcgcattgtctgagtaggtgtcattctattctggggggtggggtggggcaggacagcaagggggaggattgggaagacaatagcagg catgctggggatgcggtgggctctatggcttctgaggcggaaagaaccagctggggctctagggggtatccccacgcgcctgtagc ggcgcattaagcgcggcgggtgtggtggttacgcgcagcgtgaccgctacacttgccagcgccctagcgcccgcactagagACG TCCCGCGGGGCGGCTCTAGACATAAAAAACAAAAAAATACCCGGAGCGGGACTTGAACCC GCACAGCGCGAACGCCGAGGGATTTAGAATCCCTTGTGTCTACCGATTCCACCATCCGGG CTATTTAATTGTTGAAGAAAGAGTATACTACATAACACATATACAATTGAAAAAGAGGCTAG CAGCTTACCGGTCGGGATGGCTGCAGgatcatttatcttcactgcggagaagtttcgaacgccgaaacatgcgca ccaactttcacttctacagcgtttgaccaaaatctttgaacagaacattgtagggtgtgaaaaaatgcgcacctttaccgctagcccaa gaggg cactacaaaatctagagttgtacttcaaacgtacatgtaatcaccttgtatatactcgaaagaaaacatcaagtttctgtataaat atgagtgaaagcataatcatacattatctttcaaagactgcagAGATCTgataacaacagtgtagatgtaacaaaatcgactttgtt cccactgtacttttagctcgtacaaaatacaatatactttcatttctccgtaaacaacatgtttcccatgtaatatccttttctattttcgttccgt taccaactttacacatactttatatagctattcacttctatacactaaaaaactaagacaattttaattttgctgcctgccatatttcaatttgttat aaattcctataatttatcctattagtagctaaaaaaagatgaatgtgaatcgaatcctaagagaattgagctccaattcgccctatagtga gtcgtattacaattcactggccgtcgtttacaacgtcgtgactgggaaaaccctggcgttacccaacttaatcgccttgcagcacatccc cctttcgccagctggcgtaatagcgaagaggcccgcaccgatcgcctttcccaacagttgcgcagcctgaatggcgaatggacgcg ccctgtagcggcgcattaagcgcggcgggtgtggtggttacgcgcagcgtgaccgctacacttgccagcgccctagcgcccgctcctt tcgctttcttcccttcctttctcgccacgttcgccggctttccccgtcaagctctaaatcggggg ctccctttagggttccgatttagtgctttac ggcacctcgaccccaaaaaacttgattagggtgatggttcacgtagtgggccatcgccctgatagacggttttcgccctttgacgttgg agtccacgttctttaatagtggactcttgttccaaactggaacaacactcaaccctatctcggtctattctttgatttataagggatttgccg atttcggcctattggttaaaaaatgagctgatttaacaaaaatttaacgcgaattttaacaaaatattaacgcttacaatttcctgatgcggt attttctccttacgcatctgtgcggtatttcacaccgcatagatcggcaagtgcacaaacaatacttaaataaatactactcagtaataac ctatttcttagcattttgacgaaatttgctatttgttagagtcttttacaccatttgtctccacacctccgcttacatcaacaccaataacgcca tttaatctaagcgcatcaccaacattttctggcgtcagtccaccagctaacataaaatgtaagctttcggggctctcttgccttccaaccca gtcagaaatcgagttccaatccaaaagttcacctgtcccacctgcttctgaatcaaacaagggaataaacgaatgaggtttctgtgaag ctgcactgagtagtatgttgcagtcttttggaaatacgagtcttttaataactggcaaaccgaggaactcttggtattcttgccacgactcat ctccatgcagttggacgatatcaatgccgtaatcattgaccagagccaaaacatcctccttaggttgattacgaaacacgccaaccaa gtatttcggagtgcctgaactattttatatgcttttacaagacttgaaattttccttgcaataaccgggtcaattgttctctttctattgggcaca catataatacccagcaagtcagcatcggaatctagag cacattctgcggcctctgtgctctgcaagccgcaaactttcaccaatggac cagaactacctgtgaaattaataacagacatactccaagctgccttgtgtgcttaatcacgtatactcacgtgctcaatagtcaccaatg ccctccctcttggccctctccttttctttttcgaccgaattaattcttaatcggcaaaaaaagaaaagctccggatcaagattgtacgtaag gtgacaagctattttcaataaagaatatcttccactactgccatctggcgtcataactgcaaagtacacatatattacgatgctgttctatta aatgcttcctatattatatatatagtaatgtcgtgatctatggtg cactctcagtacaatctgctctgatgccgcatagttaagccagccccg acacccgccaacacccgctgacgcgccctgacgggcttgtctgctcccggcatccgcttacagacaagctgtgaccgtctccgggag ctgcatgtgtcagaggtttcaccgtcatcaccgaaacgcgcga

pCTCON2-FAPB2.3.6-TyrAcFRS

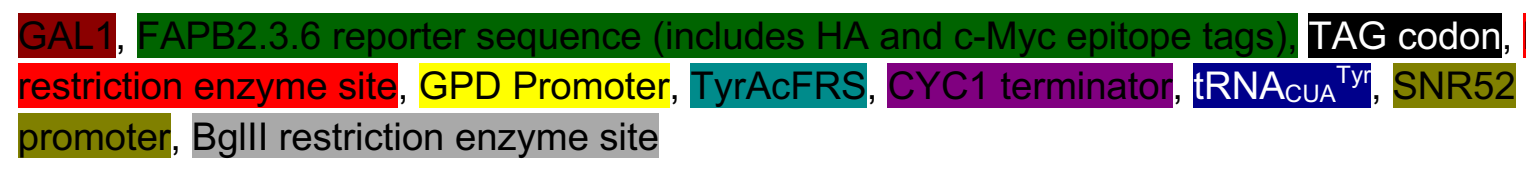


gacgaaagggcctcgtgatacgcctattttataggttaatgtcatgataataatggtttcttagacggatcgcttgcctgtaacttacacgc gcctcgtatcttttaatgatggaataatttgggaatttactctgtgtttatttatttttatgtttgtatttggattttagaaagtaaataaagaaggta gaagagttacggaatgaagaaaaaaaaataaacaaaggtttaaaaaatttcaacaaaaagcgtactttacatatatatttattagaca agaaaagcagattaaatagatatacattcgattaacgataagtaaaatgtaaaatcacaggatttcgtgtgtggtcttctacacagaca agatgaaacaattcggcattaatacctgagagcaggaagagcaagataaaaggtagtatttgttggcgatccccctagagtctttaca tcttcggaaaacaaaaactatttttctttaatttcttttttactttctattttaatttatatatttatattaaaaaatttaaattataattattttatagc acgtgatgaaaaggacccaggtgg cacttttcggggaaatgtgcgcggaacccctatttgtttattttctaaatacattcaaatatgtatc cgctcatgagacaataaccctgataaatgcttcaataatattgaaaaaggaagagtatgagtattcaacatttccgtgtcgcccttattcc ctttttgcggcatttgccttcctgttttgctcacccagaaacgctggtgaaagtaaaagatgctgaagatcagttgggtgcacgagtgg gttacatcgaactggatctcaacagcggtaagatccttgagagttttcgccccgaagaacgttttccaatgatgagcactttaaagttctg ctatgtggcgcggtattatcccgtattgacgccgggcaagagcaactcggtcgccgcatacactattctcagaatgacttggttgagtac tcaccagtcacagaaaagcatcttacggatggcatgacagtaagagaattatgcagtgctgccataaccatgagtgataacactgcg gccaacttacttctgacaacgatcggaggaccgaaggagctaaccgctttttgcacaacatgggggatcatgtaactcgccttgatcg ttgggaaccggagctgaatgaagccataccaaacgacgagcgtgacaccacgatgcctgtagcaatggcaacaacgttgcgcaa actattaactggcgaactacttactctagcttcccggcaacaattaatagactggatggaggcggataaagttgcaggaccacttctgc gctcggcccttccggctggctggtttattgctgataaatctggagccggtgagcgtgggtctcgcggtatcattgcagcactggggccag atggtaagccctcccgtatcgtagttatctacacgacggggagtcaggcaactatggatgaacgaaatagacagatcg ctgagatag gtgcctcactgattaagcattggtaactgtcagaccaagtttactcatatatactttagattgatttaaaacttcattttaatttaaaaggatct aggtgaagatccttttgataatctcatgaccaaaatccttaacgtgagttttcgttccactgagcgtcagaccccgtagaaaagatcaa aggatcttcttgagatccttttttctgcgcgtaatctgctgcttgcaaacaaaaaaaccaccgctaccagcggtggtttgtttgccggatca agagctaccaactcttttccgaaggtaactggcttcagcagagcgcagataccaaatactgttcttctagtgtagccgtagttaggcca ccacttcaagaactctgtagcaccgcctacatacctcgctctgctaatcctgttaccagtggctgctgccagtggcgataagtcgtgtctt accgggttggactcaagacgatagttaccggataaggcgcagcggtcgggctgaacggggggttcgtgcacacagcccagcttgg agcgaacgacctacaccgaactgagatacctacagcgtgagctatgagaaagcgccacgcttcccgaagggagaaaggcggac aggtatccggtaagcggcagggtcggaacaggagagcgcacgagggagcttccagggggaaacgcctggtatcttatagtcctgt cgggtttcgccacctctgacttgagcgtcgattttgtgatgctcgtcaggggggcggagcctatggaaaaacgccagcaacgcggcc ttttacggttcctggccttttgctggccttttgctcacatgttctttcctgcgttatcccctgattctgtggataaccgtattaccgcctttgagtga gctgataccgctcgccgcagccgaacgaccgagcgcagcgagtcagtgagcgaggaagcggaagagcgcccaatacgcaaac cgcctctccccgcgcgttggccgattcattaatgcagctggcacgacaggtttcccgactggaaagcgggcagtgagcgcaacgca attaatgtgagttagctcactcattaggcacccaggctttacactttatgcttccggctcgtatgttgtgtggaattgtgagcggataacaa tttcacacaggaaacagctatgaccatgattacgccaagctcgaaattaaccctcactaaagggaacaaaagctggtaccaattcctt gaattttcaaaaattcttacttttttttggatggacgcaaagaagtttaataatcatattacatggcattaccaccatatacatatccatatcta atcttacttatatgttgtggaaatgtaaagagccccattatcttagcctaaaaaaaccttctcttggaactttcagtaatacgcttaactgctc attgctatattgaagta

lattctggggtaattaatcagcgaagcg 


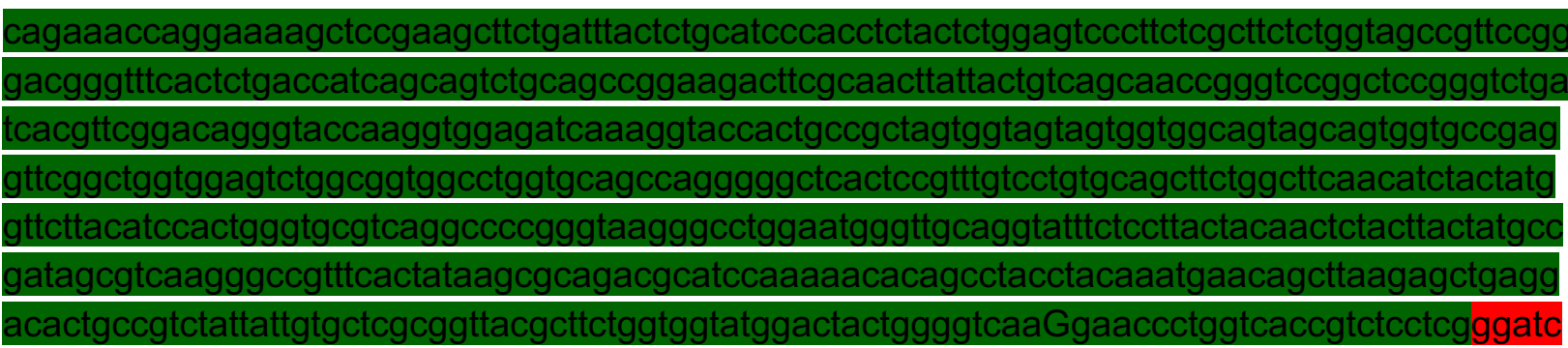

ctcgaggagctcagtttatcattatcaatactcgccatttcaaagaatacgtaa

ataattaatagtagtgatttcctaactttatttagtcaaaaaattagcctttaattctgctgtaacccgtacatgcccaaaatagggggcgg gttacacagaatatataacatcgtaggtgtctgggtgaacagtttattcctggcatccactaaatataatggagcccgctttttaagctggc atccagaaaaaaaaagaatcccagcaccaaaatattgtttcttcaccaaccatcagttcataggtccattctcttagcgcaactacag agaacaggggcacaaacaggcaaaaaacgggcacaacctcaatggagtgatgcaacctgcctggagtaaatgatgacacaag gcaattgacccacg catgtatctatctcattttcttacaccttctattaccttctgctctctctgatttggaaaaagctgaaaaaaaaggttga aaccagttccctgaaattattcccctacttgactaataagtatataaagacggtaggtattgattgtaattctgtaaatctatttcttaaacttct taaattctacttttatag ttagtctttttttagttttaaaacaccagaacttagtttcgacggattctagaactagtatggagatttCCatggca agcagtaacttgattaaacaattgcaagagcgggggctggtagcccaggtgacggacgaggaagcgttagcagagcgactggcg caaggcccgatcgcgctcATTtgcggcttcgatcctaccgctgacagcttgcatttggggcatcttgttccattgttatgcctgaaacgct tccagcaggcgggccacaagccggttgcgctggtaGGCggcgcgACGggtctgattggcgacccgagcttcaaagctgccga gcgtaagctgaacaccgaagaaactgttcaggagtgggtggacaaaatccgtaagcaggttgccccgttcctcgatttcgactgtgga gaaaactctgctatcgcggcgaacaactatgactggttcggcaatatgaatgtgctgaccttcctgcgcgatattggcaaacacttctcc gttaaccagatgatcaacaaagaagcggttaagcagcgtctcaaccgtgaaggtcaggggatttcgttcactgagtttcctacaacct gttgcagggttatggtatggcctgtgctaacaaacagtacggtgtggtgctgcaaattggtggttctgaccagtggggtaacatcacttct ggtatcgacctgacccgtcgtctgcatcagaatcaggtgtttggcctgaccgttccgctgatcactaaagcagatggcaccaaatttggt aaaactgaaggcggcgcagtctggttggatccgaagaaaaccagcccgtacaaattctaccagttctggatcaacactgcggatgc cgacgtttaccgcttcctgaagttcttcacctttatgagcattgaagagatcaacgccctggaagaagaagataaaaacagcggtaaa gcaccgcgcgcccagtatgtactggcggagcaggtgactcgtctggttcacggtgaagaaggttacaggcggcaaaacgtattacc gaatgcctgttcagcggttcttgagtgcgctgagtgaagcggacttcgaacagctggcgcaggacggcgtaccgatggttgagatgg aaaagggcgcagacctgatgcaggcactggtcgattctgaactgcaaccttcccgtggtcaggcacgtaaaactatcgcctccaatg ccatcaccattaacggtgaaaaacagtccgatcctgaatacttcttaaagaagaagatcgtctgttggtcgtttaccttactgcgtcgc ggtaaaaagaattactgtctgatttgctggaaataactcgagtcatgtaatta

ccggtaccctggaaggtgccactcccactgtcctttcctaataaaatgaggaaattgcatcgcattgtctgagtaggtgtc attctattctggggggtggggtggggcaggacagcaagggggaggattggggaagacaatagcaggcatgctggggatgcggtgg gctctatggcttctgaggcggaaagaaccagctggggctctagggggtatccccacgcgccctgtagcggcgcattaagcgcggcg ggtgtggtggttacgcgcagcgtgaccgctacacttgccagcgccctagcgcccgcactagagtcgacagacataaaaaacaaaa aaatggtgggggaaggattcgaaccttcgaagtcgatgacggcagatttagagtctgctccctttggccgetcgggaacccaccgat cattatctttcactgcggagaagtttcgaacgccgaaacatgcgcaccaactttcacttctacagcgttgaccaaaatctttgaacaga acattgtagggtgtgaaaaaatgcgcacctttaccgctagcccaagagggcactacaaaatctagagttgtacttcaaacgtacatgt aatcaccttgtatatactcgaaagaaaacatcaagtttctgtataaatatgagtgaaagcataatcatacattatctttcaaagactgcag AGATCTgataacaacagtgtagatgtaacaaaatcgactttgttcccactgtacttttagctcgtacaaaatacaatatactttcatttc tccgtaaacaacatgtttcccatgtaatatccttttctattttcgttccgttaccaactttacacatactttatatagctattcacttctatacacta aaaaactaagacaattttaattttgctgcctgccatatttcaatttgttataaattcctataatttatcctattagtagctaaaaaaagatgaat gtgaatcgaatcctaagagaattgagctccaattcgccctatagtgagtcgtattacaattcactggccgtcgtttacaacgtcgtgactg 
ggaaaaccctggcgttacccaacttaatcgccttgcagcacatcccctttcgccagctggcgtaatagcgaagaggcccgcaccga tcgcctttcccaacagttgcgcagcctgaatggcgaatggacgcgccctgtagcggcgcattaagcgcggcgggtgtggtggttacgc gcagcgtgaccgctacacttgccagcgcctagcgcccgctcctttcgctttcttcccttcctttctcgccacgttcgccggctttccccgtc aagctctaaatcgggggctccctttagggttccgatttagtgctttacggcacctcgaccccaaaaaacttgattagggtgatggttcacg tagtgggccatcgccctgatagacggttttcg ccctttgacgttggagtccacgttctttaatagtggactcttgttccaaactggaacaac actcaaccctatctcggtctattctttgatttataagggattttgccgatttcggcctattggttaaaaaatgagctgatttaacaaaaatttaa cgcgaattttaacaaaatattaacgcttacaatttcctgatgcggtattttctccttacgcatctgtgcggtattcacaccgcatagatcggc aagtgcacaaacaatacttaaataaatactactcagtaataacctatttcttagcattttgacgaaatttgctatttgttagagtctttacac catttgtctccacacctccgcttacatcaacaccaataacgccatttaatctaagcgcatcaccaacatttctggcgtcagtccaccagc taacataaaatgtaagctttcggggctctcttgccttccaacccagtcagaaatcgagttccaatccaaaagttcacctgtcccacctgct tctgaatcaaacaagggaataaacgaatgaggtttctgtgaagctgcactgagtagtatgttgcagtctttggaaatacgagtctttaat aactggcaaaccgaggaactcttggtattcttgccacgactcatctccatgcagttggacgatatcaatgccgtaatcattgaccagag ccaaaacatcctccttaggttgattacgaaacacgccaaccaagtattcggagtgcctgaactattttatatgcttttacaagacttgaa atttccttgcaataaccgggtcaattgttctcttctattgggcacacatataatacccagcaagtcagcatcggaatctagagcacattct gcggcctctgtgctctgcaagccgcaaacttcaccaatggaccagaactacctgtgaaattaataacagacatactccaagctgcctt tgtgtgcttaatcacgtatactcacgtgctcaatagtcaccaatgccctccctcttggccctctcctttctttttcgaccgaattaattcttaatc ggcaaaaaaagaaaagctccggatcaagattgtacgtaaggtgacaagctattttcaataaagaatatcttccactactgccatctgg cgtcataactgcaaagtacacatatattacgatgctgttctattaaatgcttcctatattatatatatagtaatgtcgtgatctatggtgcactct cagtacaatctgctctgatgccgcatagttaagccagccccgacacccgccaacacccgctgacgcgccctgacgggcttgtctgctc ccggcatccgcttacagacaagctgtgaccgtctccgggagctgcatgtgtcagaggtttcaccgtcatcaccgaaacgcgcga

\section{pCTCON2-FAPB2.3.6L1TAG-TyrAcFRS}

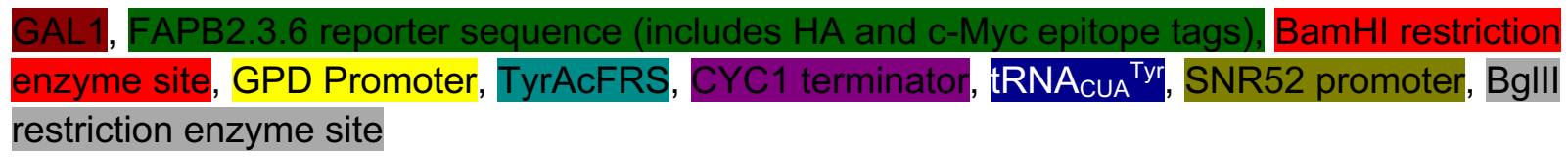

gacgaaagggcctcgtgatacgcctattttataggttaatgtcatgataataatggtttcttagacggatcgcttgcctgtaacttacacgc gcctcgtatcttttaatgatggaataatttgggaatttactctgtgtttatttattttatgttttgtatttggattttagaaagtaaataaagaaggta gaagagttacggaatgaagaaaaaaaaataaacaaaggtttaaaaaatttcaacaaaaagcgtactttacatatatattattagaca agaaaagcagattaaatagatatacattcgattaacgataagtaaaatgtaaaatcacaggatttcgtgtgtggtcttctacacagaca agatgaaacaattcggcattaatacctgagagcaggaagagcaagataaaaggtagtatttgttggcgatccccctagagtctttaca tcttcggaaaacaaaaactatttttctttaatttcttttttactttctattttaatttatatatttatattaaaaaatttaaattataattattttatagc acgtgatgaaaaggacccaggtgg cacttttcggggaaatgtgcgcggaacccctatttgtttattttctaaatacattcaaatatgtatc cgctcatgagacaataaccctgataaatgcttcaataatattgaaaaaggaagagtatgagtattcaacatttccgtgtcgcccttattcc ctttttgcggcattttgccttcctgttttgctcacccagaaacgctggtgaaagtaaaagatgctgaagatcagttgggtgcacgagtgg gttacatcgaactggatctcaacagcggtaagatccttgagagtttcgccccgaagaacgttttccaatgatgag cacttttaaagttctg ctatgtggcgcggtattatcccgtattgacgccgggcaagagcaactcggtcgccgcatacactattctcagaatgacttggttgagtac tcaccagtcacagaaaagcatcttacggatggcatgacagtaagagaattatgcagtgctgccataaccatgagtgataacactgcg gccaacttacttctgacaacgatcggaggaccgaaggagctaaccgctttttgcacaacatgggggatcatgtaactcgccttgatcg ttgggaaccggagctgaatgaagccataccaaacgacgagcgtgacaccacgatgcctgtagcaatggcaacaacgttgcgcaa actattaactggcgaactacttactctagcttcccggcaacaattaatagactggatggaggcggataaagttgcaggaccacttctgc gctcggcccttccggctggctggtttattgctgataaatctggagccggtgagcgtgggtctcgcggtatcattgcagcactggggccag atggtaagccctcccgtatcgtagttatctacacgacggggagtcaggcaactatggatgaacgaaatagacagatcgctgagatag 
gtgcctcactgattaagcattggtaactgtcagaccaagtttactcatatatactttagattgatttaaaacttcattttaatttaaaaggatct aggtgaagatccttttgataatctcatgaccaaaatcccttaacgtgagttttcgttccactgagcgtcagaccccgtagaaaagatcaa aggatcttcttgagatccttttttctgcgcgtaatctgctgcttgcaaacaaaaaaaccaccgctaccagcggtggtttgtttgccggatca agagctaccaactcttttccgaaggtaactggcttcagcagagcgcagataccaaatactgttcttctagtgtagccgtagttaggcca ccacttcaagaactctgtagcaccgcctacatacctcgctctgctaatcctgttaccagtggctgctgccagtggcgataagtcgtgtctt accgggttggactcaagacgatagttaccggataaggcgcagcggtcgggctgaacggggggttcgtgcacacagcccagcttgg agcgaacgacctacaccgaactgagatacctacagcgtgagctatgagaaagcgccacgcttcccgaagggagaaaggcggac aggtatccggtaagcggcagggtcggaacaggagagcgcacgagggagcttccagggggaaacgcctggtatcttatagtcctgt cgggtttcgccacctctgacttgagcgtcgattttgtgatgctcgtcaggggggcggagcctatggaaaaacgccagcaacgcggcc ttttacggttcctggccttttgctggccttttgctcacatgttctttcctgcgttatccctgattctgtggataaccgtattaccgcctttgagtga gctgataccgctcgccgcagccgaacgaccgagcgcagcgagtcagtgagcgaggaagcggaagagcgcccaatacgcaaac cgcctctccccgcgcgttggccgattcattaatgcagctggcacgacaggtttcccgactggaaagcgggcagtgagcgcaacgca attaatgtgagttagctcactcattaggcaccccaggctttacactttatgcttccggctcgtatgttgtgtggaattgtgagcggataacaa tttcacacaggaaacagctatgaccatgattacgccaagctcgaaattaaccctcactaaagggaacaaaagctggtaccaattcctt gaatttcaaaaattcttacttttttttggatggacgcaaagaagtttaataatcatattacatggcattaccaccatatacatatccatatcta atcttacttatatgttgtggaaatgtaaagagccccattatcttagcctaaaaaaaccttctctttggaactttcagtaatacgcttaactgctc attgctatattgaagta

atttctggggtaattaatcagcgaagcg

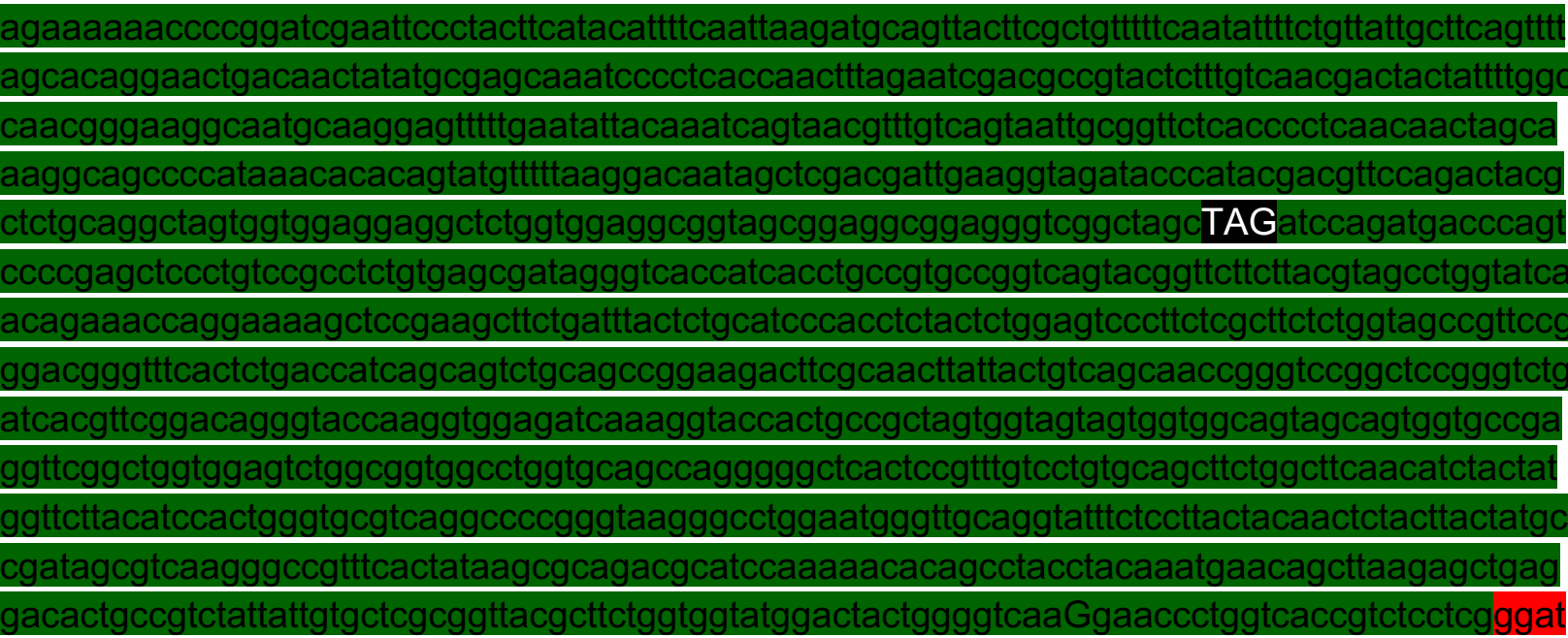

ctcgaggagctcagtttatcattatcaatactcgccatttcaaagaatacgta aataattaatagtagtgattttcctaactttatttagtcaaaaaattagcctttaattctgctgtaacccgtacatgcccaaaatagggggcg ggttacacagaatatataacatcgtaggtgtctgggtgaacagtttattcctggcatccactaaatataatggagcccgcttttaagctgg catccagaaaaaaaaagaatcccagcaccaaaatattgttttcttcaccaaccatcagttcataggtccattctcttagcgcaactacag agaacaggggcacaaacaggcaaaaaacgggcacaacctcaatggagtgatgcaacctgcctggagtaaatgatgacacaag gcaattgacccacgcatgtatctatctcattttcttacaccttctattaccttctgctctctctgatttggaaaaagctgaaaaaaaaggttga aaccagttccctgaaattattcccctacttgactaataagtatataaagacggtaggtattgattgtaattctgtaaatctatttcttaaacttct taaattctacttttatagttagtctttttttagttttaaaacaccagaacttagtttcgacggattctagaactagtatggagatttCCatggca agcagtaacttgattaaacaattgcaagagcgggggctggtagcccaggtgacggacgaggaagcgttagcagagcgactggcg caaggcccgatcgcgctcATTtgcggcttcgatcctaccgctgacagcttgcatttggggcatcttgttccattgttatgcctgaaacgct 


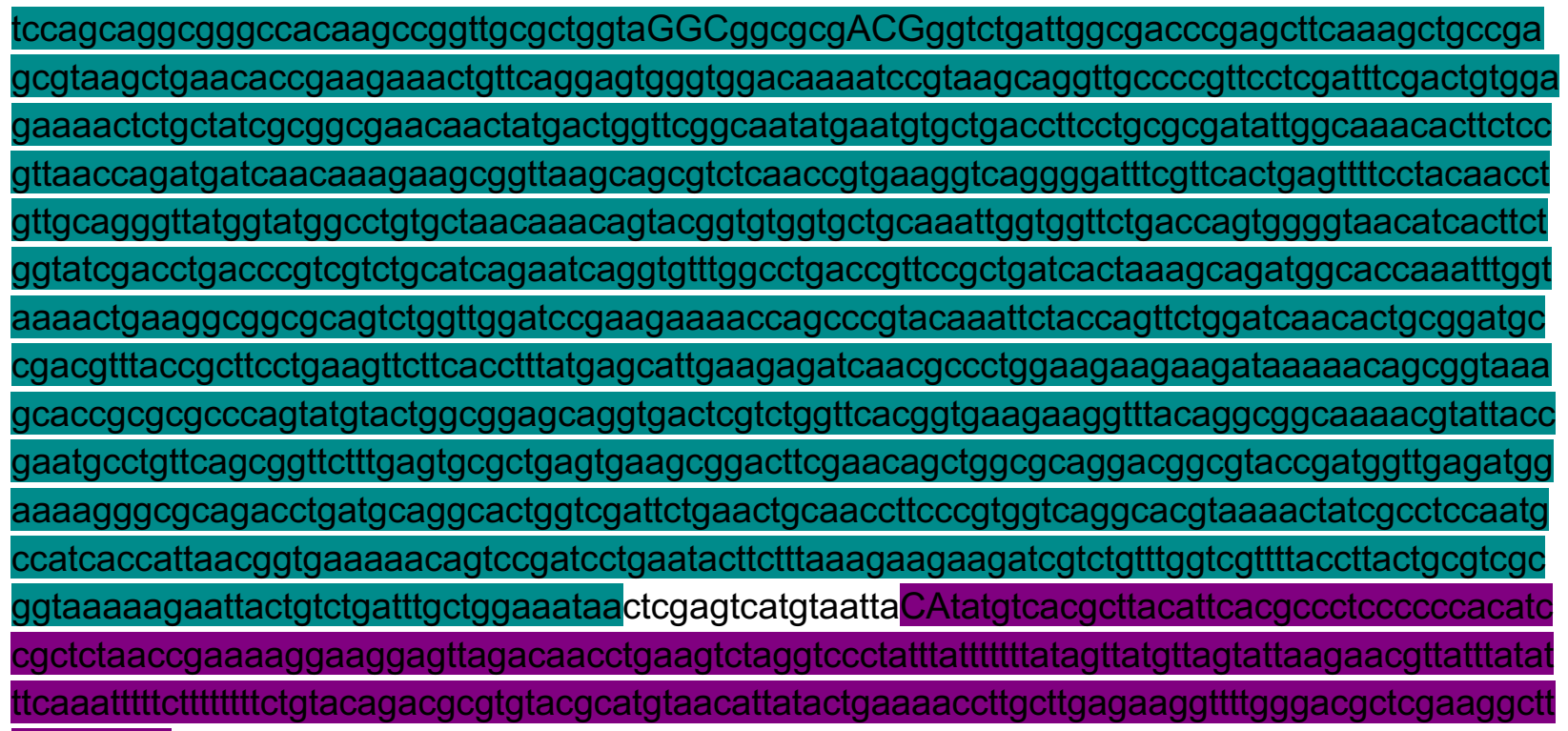

ccggtaccctggaaggtgccactcccactgtcctttcctaataaaatgaggaaattgcatcgcattgtctgagtaggtgtc attctattctggggggtggggtggggcaggacagcaagggggaggattggggaagacaatagcaggcatgctggggatgcggtgg gctctatggcttctgaggcggaaagaaccagctggggctctagggggtatccccacgcgccctgtagcggcgcattaagcgcggcg ggtgtggtggttacgcgcagcgtgaccgctacacttgccagcgccctagcgcccgcactagagtcgacagacataaaaaacaaaa aaatggtgggggaaggattcgaaccttcgaagtcgatgacggcagatttagagtctgctccctttggccgetcgggaaccccaccgat cattatctttcactgcggagaagtttcgaacgccgaaacatgcgcaccaactttcacttctacagcgttgaccaaaatctttgaacaga acattgtagggtgtgaaaaaatgcgcacctttaccgctagcccaagagggcactacaaaatctagagttgtacttcaaacgtacatgt aatcaccttgtatatactcgaaagaaaacatcaagtttctgtataaatatgagtgaaagcataatcatacattatctttcaaagactgcag AGATCTgataacaacagtgtagatgtaacaaaatcgactttgttcccactgtacttttagctcgtacaaaatacaatatacttttcatttc tccgtaaacaacatgttttcccatgtaatatccttttctatttttcgttccgttaccaactttacacatactttatatagctattcacttctatacacta aaaaactaagacaattttaattttgctgcctgccatatttcaatttgttataaattcctataatttatcctattagtagctaaaaaaagatgaat gtgaatcgaatcctaagagaattgagctccaattcgccctatagtgagtcgtattacaattcactggccgtcgtttacaacgtcgtgactg ggaaaaccctggcgttacccaacttaatcgccttgcagcacatcccctttcgccagctggcgtaatagcgaagaggcccgcaccga tcgcctttcccaacagttgcgcagcctgaatggcgaatggacgcgccctgtagcggcgcattaagcgcggcgggtgtggtggttacgc gcagcgtgaccgctacacttgccagcgccctagcgcccgctcctttcgctttcttccettcctttctcgccacgttcgccggctttccccgtc aagctctaaatcgggggetccctttagggttccgatttagtgctttacggcacctcgacccaaaaaacttgattagggtgatggttcacg tagtgggccatcgccctgatagacggttttcgccctttgacgttggagtccacgttctttaatagtggactcttgttccaaactggaacaac actcaaccctatctcggtctattctttgatttataagggattttgccgatttcggcctattggttaaaaaatgagctgatttaacaaaaatttaa cgcgaattttaacaaaatattaacgcttacaatttcctgatgcggtattttctccttacgcatctgtgcggtatttcacaccgcatagatcggc aagtgcacaaacaatacttaaataaatactactcagtaataacctatttcttagcattttgacgaaatttgctattttgttagagtctttacac catttgtctccacacctccgcttacatcaacaccaataacgccatttaatctaagcgcatcaccaacattttctggcgtcagtccaccagc taacataaaatgtaagctttcggggetctcttgccttccaacccagtcagaaatcgagttccaatccaaaagttcacctgtcccacctgct tctgaatcaaacaagggaataaacgaatgaggtttctgtgaagctgcactgagtagtatgttgcagtctttggaaatacgagtcttttaat aactggcaaaccgaggaactcttggtattcttgccacgactcatctccatg cagttggacgatatcaatgccgtaatcattgaccagag ccaaaacatcctccttaggttgattacgaaacacgccaaccaagtatttcggagtgcctgaactatttttatatgcttttacaagacttgaa atttccttgcaataaccgggtcaattgttctcttctattgggcacacatataatacccagcaagtcagcatcggaatctagagcacattct gcggcctctgtgctctgcaagccgcaaactttcaccaatggaccagaactacctgtgaaattaataacagacatactccaagctgcctt tgtgtgcttaatcacgtatactcacgtgctcaatagtcaccaatgccctccctcttggccctctcctttctttttcgaccgaattaattcttaatc ggcaaaaaaagaaaagctccggatcaagattgtacgtaaggtgacaagctatttttcaataaagaatatcttccactactgccatctgg 
cgtcataactgcaaagtacacatatattacgatgctgttctattaaatgcttcctatattatatatatagtaatgtcgtgatctatggtgcactct cagtacaatctgctctgatgccgcatagttaagccagccccgacacccgccaacacccgctgacgcgccctgacgggettgtctgctc ccggcatccgcttacagacaagctgtgaccgtctccgggagctg catgtgtcagaggtttcaccgtcatcaccgaaacgcgcga 